\title{
Impact of Ramadan diurnal intermittent fasting on the metabolic syndrome components in healthy, non-athletic Muslim people aged over 15 years: a systematic review and meta-analysis
}

\author{
'Mo'ez Al-Islam' E. Faris ${ }^{1 *}$, Haitham A. Jahrami ${ }^{2,3}$, Joud Alsibai ${ }^{1}$ and Asma A. Obaideen ${ }^{1,4}$ \\ ${ }^{1}$ Department of Clinical Nutrition and Dietetics, College of Health Sciences/Research Institute of Medical and Health Sciences \\ (RIMHS), University of Sharjah, Sharjah, United Arab Emirates \\ ${ }^{2}$ Department of Rehabilitation Services, Periphery Hospitals, Ministry of Health, Manama, Bahrain \\ ${ }^{3}$ Department of Psychiatry, College of Medicine and Medical Sciences, Arabian Gulf University, Manama, Babrain \\ ${ }^{4}$ Department of Clinical Nutrition, Faculty of Medicine and Health Sciences, University Putra Malaysia, 443400, Seri \\ Kembangan, Malaysia
}

(Submitted 15 April 2019 - Final revision received 23 August 2019 - Accepted 17 September 2019)

\section{Abstract}

Studies on the impact of Ramadan diurnal intermittent fasting (RDIF) on the metabolic syndrome (MetS) components among healthy Muslims observing Ramadan month have yielded contradictory results. This comprehensive meta-analysis aimed to obtain a more stable estimate of the effect size of fasting during Ramadan on the MetS components, examine variability among studies, assess the generalisability of reported results and perform subgroup analyses for associated factors. We searched the CINAHL, Cochrane, EBSCOhost, Google Scholar, ProQuest Medical, PubMed/MEDLINE, ScienceDirect, Scopus and Web of Science databases for relevant studies published from 1950 to March 2019. The MetS components analysed were: waist circumference (WC), systolic blood pressure (SBP), fasting plasma/serum glucose (FG), TAG, and HDL-cholesterol. We identified eighty-five studies (4326 participants in total) that were conducted in twenty-three countries between 1982 and 2019. RDIF-induced effect sizes for the MetS components were: small reductions in WC (no. of studies $K=24, N 1557$, Hedges' $g=-0 \cdot 312,95 \%$ CI $-0 \cdot 387,-0 \cdot 236)$, SBP $(K=22, N 1172$, Hedges' $g=-0 \cdot 239,95 \%$ CI $-0 \cdot 372,-0 \cdot 106)$, FG $(K=51, N 2318$, Hedges' $g=-0 \cdot 101,95 \% \mathrm{CI}-0 \cdot 260,0 \cdot 004)$ and TAG $(K=63, N 2862$, Hedges' $g=-0 \cdot 088,95 \% \mathrm{CI}-0 \cdot 171,-0 \cdot 004)$ and a small increase in HDL-cholesterol $(K=57, N 2771$, Hedges' $g=0 \cdot 150,95 \%$ CI 0.064, 0.236). We concluded that among healthy people, RDIF shows small improvement in the five MetS components: WC, SBP, TAG, FG and HDL.

Key words: Energetic restriction: Intermittent fasting: The metabolic syndrome: Meta-analysis: Ramadan: Systematic review: Time-restricted feeding

The metabolic syndrome (MetS) is considered a multiplex risk factor for atherosclerotic CVD and type 2 diabetes $^{(1)}$. Major drivers of MetS are insulin resistance, atherogenic dyslipidaemia, prothrombotic state, elevated glucose, elevated blood pressure (BP), pro-inflammatory state and excess energy intake and concomitant obesity ${ }^{(2)}$. Mounting evidence suggests that lifestyle interventions (e.g. intermittent fasting and energetic restriction ${ }^{(3)}$ or a weight reducing diet $\left.^{(4)}\right)$ and lifestyle modifications (e.g. physical exercise ${ }^{(5)}$ ) can reverse metabolic risk factors.

Ramadan is the ninth month of the Islamic lunar calendar during which healthy adult Muslims refrain from consuming food and drink from dawn to sunset. During Ramadan, the majority of Muslims throughout the world have two main meals, one immediately after sunset (suboor) and the other just before dawn (iftar). During the night hours from sunset to dawn, people are allowed to eat and drink freely but may not consume any food or drink after dawn ${ }^{(6)}$. Ramadan diurnal intermittent fasting (RDIF) represents a unique fasting pattern that involves consistent diurnal abstinence from food and drink, including water, for a fasting period of $12-18 \mathrm{~h}$ (depending on the season) over 29-30 d. In the last two decades, several published systematic reviews and meta-analyse ${ }^{(7-13)}$ and original research studies have investigated the impact of RDIF on various health

Abbreviations: BP, blood pressure; FG, fasting glucose; MetS, metabolic syndrome; RDIF, Ramadan diurnal intermittent fasting; SBP, systolic blood pressure; WC, waist circumference.

* Corresponding author: 'Mo'ez Al-Islam’ E. Faris, fax +97165057515, email mfaris@sharjah.ac.ae; moezfaris@hotmail.com 
outcomes, including risk factors for the MetS, such as body weight, body fat, lipid profile and inflammatory and oxidative stress states. The ultimate complications of the MetS, such as CVD, have also been well-investigated. However, no published works have discussed or systematically analysed the MetS components as a cluster of factors involved in the etiopathogenesis of the syndrome.

This systematic review and meta-analysis aimed to systematically summarise and analyse available scientific evidence and clarify the results of published studies regarding the impact of RDIF on the MetS components among non-diabetic, nonathletic, healthy people aged 15 years and older, who observed Ramadan fasting. The MetS components investigated in this review were elevated waist circumference (WC), elevated TAG, reduced HDL-cholesterol, elevated fasting glucose (FG) and elevated $\mathrm{BP}^{(14)}$.

The results of this analysis will expand knowledge regarding the metabolic impacts of RDIF and help to contextualise existing knowledge by examining all similar studies. The analysis of all available valid evidence pertaining to the effect of RDIF on metabolic outcomes will provide the best estimates of effect ${ }^{(15)}$. This analysis also aimed to clarify the variability between different observational and clinical studies on this topic ${ }^{(16)}$. In addition, subgroup analyses for specific MetS components were performed to explore differences in findings among countries. The findings of the present review will help to determine the generalisability of the results of identified studies, direct future researchers towards knowledge gaps that need further examination using different research models (e.g. experimental interventional trials and animal models) and inform further subgroup analyses (as appropriate).

\section{Materials and methods}

This meta-analysis used the Preferred Reporting Items for Systematic Reviews and Meta-Analyses as a guideline for reporting the findings ${ }^{(17)}$.

\section{Database searches}

Two authors (A. A. O. and M. E. F.) conducted an electronic search on the CINAHL, Cochrane, EBSCOhost, Google Scholar, ProQuest Medical, PubMed/MEDLINE, ScienceDirect, Scopus and Web of Science databases for relevant studies published from 1950 to March 2019. The search strategy included the keywords: 'Ramadan fasting' OR 'Ramadan diurnal fasting' OR 'Ramadan intermittent fasting' OR 'Ramadan model of intermittent fasting' OR 'Ramadan fast' OR 'intermittent prolonged fasting during Ramadan' AND 'metabolic syndrome' OR 'cardiometabolic risk factors' OR 'body composition' OR 'anthropometrics' OR 'waist circumference' OR 'fasting glucose' OR 'lipid profile' OR 'blood lipids' OR 'TAG' OR 'HDL' OR 'blood pressure.' Reference lists of identified studies were searched to find additional articles and reviews to ensure that all relevant publications were included in this review.

\section{Inclusion criteria}

We included observational and interventional clinical studies that examined the impact of RDIF on the MetS components. The principal criteria for study inclusion and outcomes were the MetS components as reported in the International Diabetes Federation Consensus Worldwide Definition of the Metabolic Syndrome ${ }^{(14)}$. Specific inclusion criteria for study selection were: (1) publication date between 1950 and March 2019; (2) original research articles published in the English language; (3) studies that reported numerical values (e.g., arithmetic mean with/without standard deviation (SD)) for at least one MetS component (WC, FG, TAG, HDL and systolic BP (SBP)); (4) studies that assessed the impact of RDIF on healthy people as the target population in prospective observational studies or on healthy controls in case-control, semi-experimental and experimental/interventional studies. We focused on studies that examined the effect of RDIF on the MetS components; therefore, we included studies that examined these components at least twice: pre-fasting as the baseline (e.g. few days or 1-2 weeks before Ramadan month or the first few days of Ramadan month), and post-fasting (at least 2 weeks into Ramadan month or after completion of Ramadan month). It should be noted that Islamic laws pertaining to fasting specify that premenopausal women are exempt from fasting during menstruation days; therefore, these women are not expected to complete fasting for the whole month of Ramadan. A similar exemption applies to elderly people who may find it hard to complete the whole Ramadan month and may miss some fasting days.

In all of the included studies, fasting glucose/lipid parameters were obtained from venous blood sample collected after 8-12 h of overnight fast, for the assay of all standard biochemical parameters included in glucoses/lipids profile. For the purpose of data analyses, all parameters were unified to $\mathrm{mmol} / \mathrm{l}$ rounded to two decimal places.

\section{Exclusion criteria}

Identified articles were assessed against specific exclusion criteria to eliminate potential methodological and quality issues: (1) studies that exclusively involved fasting children and adolescents $<18$ years of age; (2) studies that included patients with different diseases or conditions (including diabetes) who observed RDIF; (3) studies on the impact of RDIF on Muslim athletes that observed Ramadan fasting; (4) studies with no available full-text, even after contacting the authors; (5) studies that expressed changes in the MetS components using bar graphs and curves without reporting exact numerical values; (6) studies involving pregnant or lactating women who observed Ramadan fasting; (7) studies that reported post-Ramadan measurement after one or more months, as evidence suggests RDIF-induced biochemical variables disappear/return to pre-fasting levels after 1 month of Ramadan month cessation ${ }^{(18-20)}$; (8) case reports, abstracts, review articles, editorials and non-English-language articles and (9) unpublished, non-peer-reviewed data. Articles that met any of these criteria were excluded from the present analysis (Fig. 1). 

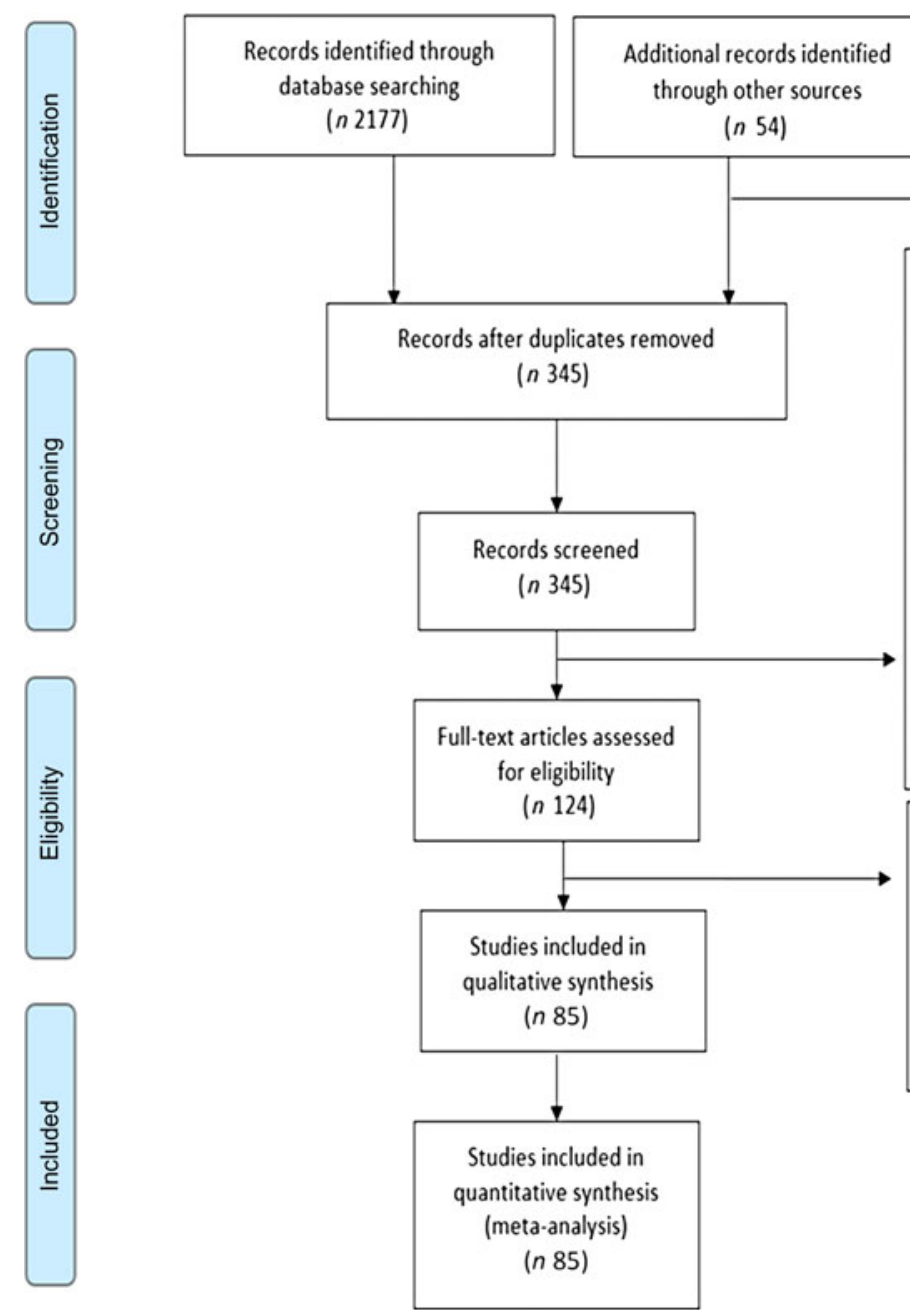

Full-text articles excluded, with reasons ( $n$ 221)

- Lack of numerical values ( $n$ 1)

- Study protocol without results $(n 1)$

- Studies on sleep during Ramadan ( $n$ 14)

- Reviews (n 43)

- Studies of neurological and psychological aspects $(n 4)$

Studies on pregnant and nursing mothers $(n 10)$

Studies on patients ( $n$ 76)

Non-English articles ( $n$ 5)

- Food patterns, knowledge and attitude studies ( $n$ 8)

- Commentaries and letters ( $n$ 9)

Studies on fasting children $(n 4)$

- Studies on athletes ( $n$ 33)

- Animal model of Ramadan fasting ( $n$ 6)

Abstracts ( $n$ 7)

Full-text articles excluded, with reasons ( $n$ 39)

- Post-fasting measurement after more than one month of Ramadan ( $n$ 3)

- Age group less than 18 years $(n 3)$

- Studies on patients $(n 4)$

- Studies on athlete people ( $n$ 3)

- Lack of numerical values $(n)$

Lacking at least one Mets component (n 23)

Fig. 1. Preferred Reporting Items for Systematic Reviews and Meta-Analyses flow chart for the selection of publications included in the systematic review and metaanalysis. MetS, metabolic syndrome.

\section{Main outcomes and measures}

The principal outcome of this review was to report the effect of RDIF on effect size changes in the MetS components (WC, FG, TAG, HDL and SBP). SBP was chosen as it is a major component of BP. Two authors (A. A. O. and M. E. F.) independently screened the titles and abstracts of identified studies to assess the studies for eligibility. The first step of screening was examining all titles and abstracts to exclude irrelevant publications. Two authors (M. E. F. and J. A.) performed this initial screening, which was validated by another author (A. A. O). Any conflicts in opinions regarding study eligibility were resolved through dialogue with a fourth author (H. A. J.) to reach consensus. To standardise data extraction, the review team systematically collected and coded data for study characteristics (e.g. title, year, country, sample size and participants' characteristics such as age, sex or proportion of males) and the main findings for the MetS components before and after RDIF.

\section{Estimating fasting time length}

Ramadan month as presented in the lunar calendar was matched with the Gregorian calendar using a time and date website (https://www.timeanddate.com/holidays/us/ramadan-begins). The daily length of fasting during Ramadan month was calculated using the sunrise and sunset times reported for that month for the city/country of each included study (https://www. timeanddate.com/sun/@8469718). Time points for Ramadan fasting are the call to prayer (Athan) for Fajr (abstinence or Imsak time, end of pre-fasting meal time or suboor) and sunset or Maghrib (breakfast or Iftar meal time) prayer times. The sunrise prayer time is declared by Fajr Athan to be about mean of $80 \mathrm{~min}$ before the real sunrise time, as recorded in the Islamic calendar for prayer times. Therefore, the actual length of fasting time was calculated by adding $80 \mathrm{~min}$ to the time between the sunrise and sunset time points. Details of the pre-dawn Fajr and sunset Maghrib prayer time points on the Islamic calendar are available on the Islamic Finder website for 
Sharjah city, United Arab Emirates (https://www.islamicfinder. org/world/united-arab-emirates/292672/sharjah-prayer-times/). This showed that the length of fasting time for a specific day (time between the Fajr and Maghrib prayer times) was $787 \mathrm{~min}$ (approximately $13 \mathrm{~h}$ ), which was close to the length of fasting time calculated using the solar calendar (sunrise and sunset time points).

\section{Data synthesis and statistical analyses}

Combined means were computed when studies included subgroups (e.g. normal body weight, overweight, obese), with different means and SD reported for each subgroup. $P$-values for the combined subgroup means were calculated. All descriptive and inferential tests were performed using Stata software (Stata, M.P., 15.0.: StataCorp, 2017).

We performed a series of one-group (pre-post) metaanalyses using a pre- and post-means model, sample size and $P$-values (paired groups). Hedges' $g$ was used to measure the effect size. Hedges' $g$ is an important measure of corrected effect size that is sensitive to even small samples $(<20)$. An effect size is a quantitative measure of the magnitude of a change between two groups or one group under two conditions, for example, before and after. Details on Hedges' $g$ formula can be obtained from the original publication by Larry V. Hedges ${ }^{(21)}$. An effect size of $\leq 0 \cdot 2$ was considered a small effect, an effect size approximately 0.5 a medium effect and an effect size approximately 0.8 a large effect. A Hedges' $g$ value of one indicates the two groups differ by one SD, a $\mathrm{g}$ value of two indicates they differ by two SD, and so on. $\mathrm{SD}$ are equivalent to $z$-scores $(1 \mathrm{SD}=1 z$-score). In addition to Hedges' g, we used forest plots to graphically present the results and illustrate point estimates for effect sizes and $95 \%$ CI.

Random effects modelling was used for all analyses. By using random effects modelling, we therefore assume that there is not only one true effect size, rather a distribution of true effect sizes. We therefore wanted in this project to estimate the mean of this distribution of true effect sizes. $I^{2}$ statistics were used to assess the heterogeneity between included studies, and $\tau^{2}$ statistics were used to assess heterogeneity within studies.

Comprehensive Meta-Analysis version $2^{(22)}$ was used for all analyses. To confirm that our meta-analysis findings were not driven by a single study, we conducted leave-one-out sensitivity analyses (sensitivity analysis) by iteratively eliminating one study at a time. Moderator analysis was performed using subgroup analyses for categorical variables (country) and meta-regression for integer or decimal variables (age, percentage of male participants and fasting time per $\mathrm{d}$ ).

Computing $I^{2}$ and $\tau^{2}$ statistics were particularly important to examine heterogeneity due to explainable causes, for example, timing of data collection before Ramadan month and post-fasting.

The algorithm used to estimate a random effects metaregression model was obtained using methods of moments ${ }^{(23)}$. $\beta$-coefficients and $P$-values resulting from modelling were reported. Graphical plots were used to visually aid the interpretation of the results. Funnel-plot-based analysis was used to detect publication bias, and the nonparametric trim and fill method was used to confirm the findings ${ }^{(24)}$. Finally, subgroup analysis for each MetS component was performed to investigate differences among countries. We performed this analysis if three or more studies were available from any given country.

\section{Critical appraisal of studies (quality assessment)}

Two reviewers (M. E. F. and A. A. O.) independently assessed the methodological quality of studies using a standardised checklist consisting of six items in terms of sample size and sampling technique, standardisation of data collection, appropriateness of statistical analyses, quality of reporting results and generalisability. The appraisal scores range between 0 and 6 , with scores of 0-2 corresponds to low quality, 3-4 medium quality and 5-6 high quality. Quality score was set for each study by consensus after discussion (see online Supplementary Material 2).

\section{Results}

Eighty-five studies with a total of 4326 participants were included in this meta-analysis. Details of the sample size, participants' sex and age, study design and major findings related to the MetS components for the included studies are shown in Table 1. All included studies used a pre-post design to report changes in the MetS components. Approximately $64 \%$ of participants were male, and the median age was 31.5 years (range $15-80$ years). The mean length of fasting during Ramadan across all studies was 828 (sD 90) $\mathrm{min} / \mathrm{d}$ (range 668-1038 min).

Quality assessment of the analysed studies indicated that $15 \cdot 3 \%(13 / 85)$ were of low quality, while $84.7 \%(72 / 85)$ were of medium quality.

\section{Meta-analysis of the metabolic syndrome components}

Table 2 summarises the number of studies $(K)$, number of participants $(N)$, participants' mean age, percentage of male participants and fasting time $(\mathrm{min} / \mathrm{d})$. Visual inspection of the funnel plots indicated no bias in any of the included studies (online Supplementary Fig. S1-S5). Meta-analytic pooling WC, FG, TAG, HDL and SBP was performed, and the results expressed as $K, N$, Hedges' $g$, 95\% CI and $I^{2}$ : WC $(K=24$, $N$ 1557, Hedges' $g=-0.312,95 \%$ CI $-0.387,-0.236$, $I^{2}=49 \%$; Fig. 2); FG $(K=51, N 2318$, Hedges' $g=-0 \cdot 101$, $95 \%$ CI $-0.260,0.004, I^{2}=26.6 \%$; Fig. 3); TAG $(K=63$, $N$ 2862, Hedges' $g=-0.088,95 \%$ CI $-0 \cdot 171,-0 \cdot 004$, $I^{2}=78 \%$; Fig. 4); HDL $(K=57, N$ 2771, Hedges' $g=0 \cdot 150$, $95 \%$ CI $0.064,0.236, I^{2}=79 \% ;$ Fig. 5) and SBP $(K=22$, $N$ 1172, Hedges' $g=-0.239$, $95 \%$ CI $-0.372,-0 \cdot 106$, $I^{2}=78 \%$; Fig. 6). Sensitivity analyses were performed for the MetS components by removing one study at a time to determine if the pooled effect size for each component was arbitrary or influenced by a single study.

\section{Moderator analysis for the metabolic syndrome components}

Table 3 shows the results of the moderator analysis for each MetS component. We also performed subgroup analysis for specific MetS components for countries from which three or 


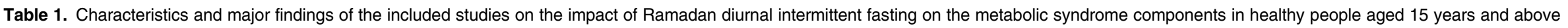

\begin{tabular}{|c|c|c|c|c|c|c|c|c|c|c|c|c|c|}
\hline \multirow{2}{*}{$\begin{array}{l}\text { Study, publication } \\
\text { year }\end{array}$} & \multirow[b]{2}{*}{ Country } & \multicolumn{2}{|c|}{ Sample size } & \multicolumn{2}{|c|}{ Age (years) } & \multirow[b]{2}{*}{ Study design } & \multirow{2}{*}{$\begin{array}{l}\text { Examined } \\
\text { markers }\end{array}$} & \multicolumn{2}{|c|}{ Before Ramadan† } & \multicolumn{2}{|c|}{ End of Ramadan† } & \multirow{2}{*}{$\begin{array}{l}\text { Results (after } \\
\text { Ramadan month } \\
\text { compared with before) }\end{array}$} & \multirow{2}{*}{$\begin{array}{l}\text { Total } \\
\text { quality } \\
\text { score }\end{array}$} \\
\hline & & $n$ & $\%$ Male & Mean & Range & & & Mean & SD & Mean & SD & & \\
\hline Fedail et al., $1982^{(25)}$ & Sudan & 24 & 83.3 & 30 & $21-40$ & $\begin{array}{l}\text { Prospective } \\
\text { observational }^{*}\end{array}$ & TAG & $1.32 \mathrm{mmol} / \mathrm{l}$ & 0.22 & $1.27 \mathrm{mmol} / \mathrm{l}$ & 0.17 & $\begin{array}{l}\text { Non-significant } \\
\text { decrease in TAG }\end{array}$ & 4 \\
\hline $\begin{array}{l}\text { Azizi \& Rasouli, } \\
1987^{(26)}\end{array}$ & Iran & 9 & 100 & 35 & $23-54$ & $\begin{array}{l}\text { Prospective } \\
\text { observational }^{*}\end{array}$ & $\mathrm{FG}$ & $82 \mathrm{mg} / \mathrm{dl}$ & 4.4 & 84 mg/dl & $5 \cdot 2$ & $\begin{array}{l}\text { No significant change } \\
\text { in FG }\end{array}$ & 4 \\
\hline El Ati et al., 1995(27) & Tunisia & 16 & 0 & & 25-39 & $\begin{array}{l}\text { Prospective } \\
\text { observational }^{*}\end{array}$ & $\begin{array}{l}\text { TAG } \\
\text { FG }\end{array}$ & $\begin{array}{l}0.68 \mathrm{mmol} / \mathrm{l} \\
4.86 \mathrm{mmol} / \mathrm{l}\end{array}$ & $\begin{array}{l}0.05 \\
0.39\end{array}$ & $\begin{array}{l}0.66 \mathrm{mmol} / \mathrm{l} \\
5.21 \mathrm{mmol} / \mathrm{l}\end{array}$ & $\begin{array}{l}0.07 \\
0.62\end{array}$ & $\begin{array}{l}\text { No significant change } \\
\text { in TAG or FG }\end{array}$ & 4 \\
\hline Adlouni et al., $1997^{(28)}$ & Morocco & 32 & 100 & & $25-50$ & $\begin{array}{l}\text { Prospective } \\
\text { observational }^{*}\end{array}$ & $\begin{array}{l}\text { HDL } \\
\text { TAG } \\
\text { FG }\end{array}$ & $\begin{array}{l}0.91 \mathrm{mmol} / / \\
1 \mathrm{mmol} / \mathrm{l} \\
5.1 \mathrm{mmol} / \mathrm{l}\end{array}$ & $\begin{array}{l}0.21 \\
0.42 \\
0.5\end{array}$ & $\begin{array}{l}1.04 \mathrm{mmol} / / \\
0.7 \mathrm{mmol} / \mathrm{l} \\
4.38 \mathrm{mmol} / \mathrm{l}\end{array}$ & $\begin{array}{l}0.08 \\
0.28 \\
0.39\end{array}$ & $\begin{array}{l}\text { Significant decrease in } \\
\text { serum TAG and FG } \\
\text { and a significant } \\
\text { increase in HDL }\end{array}$ & 4 \\
\hline Bilto, $1998^{(29)}$ & Jordan & 74 & 81 & & $20-48$ & $\begin{array}{l}\text { Prospective } \\
\text { observational }^{*}\end{array}$ & $\begin{array}{l}\text { HDL } \\
\text { TAG } \\
\text { FG }\end{array}$ & $\begin{array}{l}1.3 \mathrm{mmol} / \mathrm{l} \\
1.35 \mathrm{mmol} / \mathrm{l} \\
5.4 \mathrm{mmol} / \mathrm{l}\end{array}$ & $\begin{array}{l}0.33 \\
0.68 \\
2 \cdot 21\end{array}$ & $\begin{array}{l}1.07 \mathrm{mmol} / \mathrm{l} \\
0.82 \mathrm{mmol} / \mathrm{l} \\
4.73 \mathrm{mmol} / \mathrm{l}\end{array}$ & $\begin{array}{l}0.19 \\
0.33 \\
0.5\end{array}$ & $\begin{array}{l}\text { HDL decreased } \\
\text { significantly }\end{array}$ & 4 \\
\hline Maislos et al., 1998 ${ }^{(30)}$ & Israel & 22 & 64 & 24 & $20-45$ & $\begin{array}{l}\text { Prospective } \\
\text { observational }^{*}\end{array}$ & $\begin{array}{l}\text { HDL } \\
\text { TAG } \\
\text { FG }\end{array}$ & $\begin{array}{l}0.91 \mathrm{mmol} / \mathrm{l} \\
1.3 \mathrm{mmol} / \mathrm{l} \\
4.27 \mathrm{mmol} / \mathrm{l}\end{array}$ & $\begin{array}{l}0.28 \\
0.7 \\
0.66\end{array}$ & $\begin{array}{l}1.13 \mathrm{mmol} / \mathrm{l} \\
1.3 \mathrm{mmol} / \mathrm{l} \\
4.44 \mathrm{mmol} / \mathrm{l}\end{array}$ & $\begin{array}{l}0.27 \\
1.1 \\
0.33\end{array}$ & $\begin{array}{l}\text { Only HDL increased } \\
\text { significantly }\end{array}$ & 4 \\
\hline $\begin{array}{l}\text { Mahboob et al., } \\
1999^{(31)}\end{array}$ & Iran & 35 & 100 & 25 & $19-33$ & $\begin{array}{l}\text { Prospective } \\
\text { observational }^{*}\end{array}$ & $\begin{array}{l}\text { HDL } \\
\text { TAG }\end{array}$ & $\begin{array}{l}49.53 \mathrm{mg} / \mathrm{dl} \\
83.5 \mathrm{mg} / \mathrm{dl}\end{array}$ & $\begin{array}{r}7.48 \\
30.64\end{array}$ & $\begin{array}{l}51.35 \mathrm{mg} / \mathrm{dl} \\
76.54 \mathrm{mg} / \mathrm{dl}\end{array}$ & $\begin{array}{l}11 \cdot 71 \\
17 \cdot 59\end{array}$ & $\begin{array}{l}\text { No significant change } \\
\text { in HDL or TAG }\end{array}$ & 4 \\
\hline Akanji et al., 2000(32) & Kuwait & 49 & NR & $47 \cdot 6$ & $10 \cdot 8$ & $\begin{array}{l}\text { Prospective } \\
\text { observational }^{*}\end{array}$ & $\begin{array}{l}\text { HDL } \\
\text { TAG } \\
\text { FG }\end{array}$ & $\begin{array}{l}1.16 \mathrm{mmol} / \mathrm{l} \\
2.7 \mathrm{mmol} / \mathrm{l} \\
5.59 \mathrm{mmol} / \mathrm{l}\end{array}$ & $\begin{array}{l}0.32 \\
1.5 \\
1\end{array}$ & $\begin{array}{l}1.13 \mathrm{~mol} / \mathrm{l} \\
2.7 \mathrm{mmol} / \mathrm{l} \\
5.9 \mathrm{mmol} / \mathrm{l}\end{array}$ & $\begin{array}{l}0.36 \\
2 \cdot 4 \\
1 \cdot 2\end{array}$ & $\begin{array}{l}\text { No significant change } \\
\text { in } \mathrm{HDL}, \mathrm{TAG} \text { or } \mathrm{FG}\end{array}$ & 2 \\
\hline Asgary et al., 2000(33) & Iran & 46 & 100 & & $30-60$ & Cross-sectional & $\begin{array}{l}\text { TAG } \\
\text { FG }\end{array}$ & $\begin{array}{l}209.8 \mathrm{mg} / \mathrm{dl} \\
92.57 \mathrm{mg} / \mathrm{dl}\end{array}$ & $\begin{array}{l}71.65 \\
33.73\end{array}$ & $\begin{array}{l}193.00 \mathrm{mg} / \mathrm{dl} \\
90.22 \mathrm{mg} / \mathrm{dl}\end{array}$ & $\begin{array}{l}57.15 \\
22.09\end{array}$ & $\begin{array}{l}\text { TAG decreased } \\
\text { significantly; FG } \\
\text { decreased, but was } \\
\text { not significant }\end{array}$ & 4 \\
\hline Qujeq et al., 2002(34) & Iran & 83 & 69 & $\begin{array}{r}34.25 \\
(\mathrm{SD} 9.8)\end{array}$ & $21-55$ & $\begin{array}{l}\text { Prospective } \\
\text { observational }^{*}\end{array}$ & HDL & $\begin{array}{l}\text { Male: } 1.09 \mathrm{mmol} / \mathrm{l} \\
\text { Female: } 1.13 \mathrm{mmol} / \mathrm{l}\end{array}$ & $\begin{array}{l}0.11 \\
0.12\end{array}$ & $\begin{array}{l}\text { Male: } 1.74 \mathrm{mmol} / \mathrm{l} \\
\text { Female: } 1.81 \mathrm{mmol} / \mathrm{l}\end{array}$ & $\begin{array}{l}0.18 \\
0.19\end{array}$ & $\begin{array}{l}\text { Statistically significant } \\
\text { elevation in HDL }\end{array}$ & 4 \\
\hline Ramadan, $2002^{(35)}$ & Kuwait & 16 & 100 & NR & & $\begin{array}{l}\text { Prospective } \\
\text { observational }^{*}\end{array}$ & $\begin{array}{l}\text { TAG } \\
\text { FG }\end{array}$ & $\begin{array}{l}1.4 \mathrm{mmol} / \mathrm{l} \\
5 \mathrm{mmol} / \mathrm{l}\end{array}$ & $\begin{array}{l}0.2 \\
0.1\end{array}$ & $\begin{array}{l}1.3 \mathrm{mmol} / \mathrm{l} \\
5.5 \mathrm{mmol} / \mathrm{l}\end{array}$ & $\begin{array}{l}0.1 \\
0.1\end{array}$ & $\begin{array}{l}\text { No significant changes } \\
\text { in TAG and FG }\end{array}$ & 2 \\
\hline $\begin{array}{l}\text { Afrasiabi et al., } \\
2003^{(36)}\end{array}$ & Iran & 16 & 100 & NR & & $\begin{array}{l}\text { Prospective } \\
\text { observational }^{*}\end{array}$ & $\begin{array}{l}\text { HDL } \\
\text { TAG }\end{array}$ & $\begin{array}{l}43.2 \mathrm{mg} / \mathrm{dl} \\
235.7 \mathrm{mg} / \mathrm{dl}\end{array}$ & $\begin{array}{r}2 \cdot 2 \\
36 \cdot 9\end{array}$ & $\begin{array}{l}45 \mathrm{mg} / \mathrm{dl} \\
171.1 \mathrm{mg} / \mathrm{dl}\end{array}$ & $\begin{array}{r}2 \cdot 1 \\
25 \cdot 9\end{array}$ & $\begin{array}{l}\text { Significant reduction in } \\
\text { TAG }\end{array}$ & 2 \\
\hline $\begin{array}{l}\text { Fakhrzadeh et al., } \\
2003^{(37)}\end{array}$ & Iran & 91 & 55 & $\begin{array}{c}19.9 \\
(S D 1.8)\end{array}$ & & $\begin{array}{l}\text { Prospective } \\
\text { observational }^{*}\end{array}$ & $\begin{array}{l}\text { WC } \\
\text { HDL } \\
\text { TAG } \\
\text { FG } \\
\text { SBP }\end{array}$ & $\begin{array}{l}\text { Male: } 74.2 \mathrm{~cm} \\
\text { Female: } 81.2 \mathrm{~cm} \\
\text { Male: } 39.9 \mathrm{mg} / \mathrm{dl} \\
\text { Female: } 48.1 \mathrm{mg} / \mathrm{dl} \\
\text { Male: } 118.6 \mathrm{mg} / \mathrm{dl} \\
\text { Female: } 130 \mathrm{mg} / \mathrm{dl} \\
\text { Male: } 87.5 \mathrm{mg} / \mathrm{dl} \\
\text { Female: } 89.7 \mathrm{mg} / \mathrm{dl} \\
\text { Male: } 117.7 \mathrm{mg} / \mathrm{dl} \\
\text { Female: } 103.8 \mathrm{mg} / \mathrm{dl}\end{array}$ & \begin{tabular}{r|}
$10 \cdot 4$ \\
2 \\
$7 \cdot 1$ \\
$10 \cdot 2$ \\
$45 \cdot 6$ \\
$85 \cdot 1$ \\
$8 \cdot 8$ \\
$9 \cdot 3$ \\
$11 \cdot 4$ \\
$12 \cdot 1$
\end{tabular} & $\begin{array}{l}\text { Male: } 75 \mathrm{~cm} \\
\text { Female: } 78.1 \mathrm{~cm} \\
\text { Male: } 48.3 \mathrm{mg} / \mathrm{dl} \\
\text { Female: } 62.9 \mathrm{mg} / \mathrm{dl} \\
\text { Male: } 74.4 \mathrm{mg} / \mathrm{dl} \\
\text { Female: } 105.2 \mathrm{mg} / \mathrm{dl} \\
\text { Male: } 60.8 \mathrm{mg} / \mathrm{dl} \\
\text { Female: } 65.7 \mathrm{mg} / \mathrm{dl} \\
\text { Male: } 117.2 \mathrm{mg} / \mathrm{dl} \\
\text { Female: } 103.7 \mathrm{mg} / \mathrm{dl}\end{array}$ & $\begin{array}{c}6 \\
12 \cdot 5 \\
7 \cdot 2 \\
7 \cdot 2 \\
18 \cdot 3 \\
1 \\
64 \cdot 7 \\
6 \cdot 5 \\
18 \cdot 4 \\
10 \cdot 6 \\
11 \cdot 2\end{array}$ & $\begin{array}{l}\text { Significant reduction in } \\
\text { WC in women; FG } \\
\text { decreased } \\
\text { significantly in both } \\
\text { men and women; } \\
\text { Serum TAG } \\
\text { decreased and HDL } \\
\text { increased } \\
\text { significantly }\end{array}$ & 4 \\
\hline Kassab et al., 2003 ${ }^{(38)}$ & Bahrain & 44 & 0 & & $18-45$ & $\begin{array}{l}\text { Prospective } \\
\text { observational }^{*}\end{array}$ & $\begin{array}{l}\text { WC } \\
\text { TAG } \\
\text { FG }\end{array}$ & $\begin{array}{l}\text { Lean: } 72.2 \mathrm{~cm} \\
\text { Obese: } 93.1 \mathrm{~cm} \\
\text { Lean: } 0.72 \mathrm{mmol} / / \\
\text { Obese: } 0.95 \mathrm{mmol} / / \\
\text { Lean: } 5.27 \mathrm{mmol} / / \\
\text { Obese: } 5.81 \mathrm{mmol} / /\end{array}$ & $\begin{array}{l}1 \\
2 \cdot 7 \\
0.05 \\
0.16 \\
0.08 \\
0.47\end{array}$ & $\begin{array}{l}\text { Lean: } 71.5 \mathrm{~cm} \\
\text { Obese: } 89.2 \mathrm{~cm} \\
\text { Lean: } 0.77 \mathrm{mmol} / / \\
\text { Obese: } 1.04 \mathrm{mmol} / / \\
\text { Lean: } 5.06 \mathrm{mmol} / / \\
\text { Obese: } 5.84 \mathrm{mmol} / /\end{array}$ & $\begin{array}{l}1 \\
2.5 \\
0.03 \\
0.21 \\
0.15 \\
0.61\end{array}$ & $\begin{array}{l}\text { No significant change } \\
\text { in WC, TAG or FG }\end{array}$ & 4 \\
\hline Larijani et al., $2003^{(39)}$ & Iran & 115 & 58 & $\begin{array}{l}21 \cdot 2 \\
(\mathrm{SD} 4 \cdot 3)\end{array}$ & $15-45$ & $\begin{array}{l}\text { Prospective } \\
\text { observational }^{*}\end{array}$ & $\mathrm{FG}$ & $88.4 \mathrm{mg} / \mathrm{dl}$ & 9 & $62.9 \mathrm{mg} / \mathrm{dl}$ & $7 \cdot 7$ & Significant drop in $\mathrm{FG}$ & 4 \\
\hline
\end{tabular}




\section{N British Journal of Nutrition}

Table 1. Continued

\begin{tabular}{|c|c|c|c|c|c|c|c|c|c|c|c|c|c|}
\hline \multirow{2}{*}{$\begin{array}{l}\text { Study, publication } \\
\text { year }\end{array}$} & \multirow[b]{2}{*}{ Country } & \multicolumn{2}{|c|}{ Sample size } & \multicolumn{2}{|c|}{ Age (years) } & \multirow[b]{2}{*}{ Study design } & \multirow{2}{*}{$\begin{array}{c}\text { Examined } \\
\text { markers }\end{array}$} & \multicolumn{2}{|c|}{ Before Ramadan† } & \multicolumn{2}{|c|}{ End of Ramadan† } & \multirow{2}{*}{$\begin{array}{l}\text { Results (after } \\
\text { Ramadan month } \\
\text { compared with before) }\end{array}$} & \multirow{2}{*}{$\begin{array}{l}\text { Total } \\
\text { quality } \\
\text { score }\end{array}$} \\
\hline & & $n$ & $\%$ Male & Mean & Range & & & Mean & SD & Mean & SD & & \\
\hline Kassab et al., 2004(40) & Bahrain & 46 & 0 & $\begin{array}{l}22 \\
(\mathrm{SD} 2)\end{array}$ & $18-45$ & $\begin{array}{l}\text { Prospective } \\
\text { observational }^{*}\end{array}$ & $\begin{array}{l}\text { WC } \\
\text { TAG } \\
\text { FG }\end{array}$ & $\begin{array}{l}77.7 \mathrm{~cm} \\
0.79 \mathrm{mmol} / \mathrm{l} \\
5.41 \mathrm{mmol} / \mathrm{l}\end{array}$ & $\begin{array}{l}1.6 \\
0.06 \\
0.14\end{array}$ & $\begin{array}{l}75.8 \mathrm{~cm} \\
0.84 \mathrm{mmol} / \mathrm{l} \\
5.27 \mathrm{mmol} / \mathrm{l}\end{array}$ & $\begin{array}{l}1.5 \\
0.06 \\
0.2\end{array}$ & $\begin{array}{l}\text { No significant change } \\
\text { in WC, TAG or FG }\end{array}$ & 4 \\
\hline $\begin{array}{l}\text { Rahman et al., } \\
\text { 2004(41) }\end{array}$ & Bangladesh & 20 & 100 & $\begin{array}{c}38.27 \\
(\mathrm{sD} 4.07)\end{array}$ & & $\begin{array}{l}\text { Prospective } \\
\text { observational }^{*}\end{array}$ & $\begin{array}{l}\text { HDL } \\
\text { TAG } \\
\text { FG } \\
\text { SBP }\end{array}$ & $\begin{array}{l}38.14 \mathrm{mg} / \mathrm{dl} \\
146.66 \mathrm{mg} / \mathrm{dl} \\
105.3 \mathrm{mg} / \mathrm{dl} \\
124.3 \mathrm{mmHg}\end{array}$ & $\begin{array}{l}7 \cdot 4 \\
72 \cdot 78 \\
14 \cdot 1 \\
13 \cdot 9\end{array}$ & $\begin{array}{l}46.71 \mathrm{mg} / \mathrm{dl} \\
131.04 \mathrm{mg} / \mathrm{dl} \\
85.6 \mathrm{mg} / \mathrm{dl} \\
111.8 \mathrm{mmHg}\end{array}$ & $\begin{array}{l}14 \cdot 33 \\
41 \cdot 47 \\
12 \cdot 4 \\
10 \cdot 8\end{array}$ & $\begin{array}{l}\text { SBP and FG } \\
\text { significantly } \\
\text { decreased; HDL } \\
\text { increased } \\
\text { significantly }\end{array}$ & 4 \\
\hline Yucel et al., 2004(42) & Turkey & 38 & 55 & $\begin{array}{c}32.5 \\
(\mathrm{sD} 12.5)\end{array}$ & $20-45$ & Prospective & WC & $83.29 \mathrm{~cm}$ & $13 \cdot 21$ & $83.44 \mathrm{~cm}$ & $12 \cdot 82$ & $\begin{array}{l}\text { No significant change } \\
\text { in WC }\end{array}$ & 4 \\
\hline $\begin{array}{l}\text { Aksungar et al., } \\
2005^{(43)}\end{array}$ & Turkey & 24 & 50 & & $21-35$ & $\begin{array}{l}\text { Prospective } \\
\quad \text { observational }^{*}\end{array}$ & $\begin{array}{l}\mathrm{HDL} \\
\mathrm{TAG}\end{array}$ & $\begin{array}{l}\text { Male: } 49 \mathrm{mg} / \mathrm{dl} \\
\text { Female: } 57.4 \mathrm{mg} / \mathrm{dl} \\
\text { Male: } 76.27 \mathrm{mg} / \mathrm{dl} \\
\text { Female: } 66.82 \mathrm{mg} / \mathrm{dl}\end{array}$ & $\begin{array}{l}15 \cdot 25 \\
13 \cdot 63 \\
29 \cdot 04 \\
23 \cdot 53\end{array}$ & $\begin{array}{l}\text { Male: } 56 \mathrm{mg} / \mathrm{dl} \\
\text { Female: } 66.5 \mathrm{mg} / \mathrm{dl} \\
\text { Male: } 76.68 \mathrm{mg} / \mathrm{dl} \\
\text { Female: } 68.66 \mathrm{mg} / \mathrm{dl}\end{array}$ & $\begin{array}{l}16 \cdot 31 \\
11.79 \\
27 \cdot 08 \\
15 \cdot 49\end{array}$ & $\begin{array}{l}\text { HDL increased } \\
\text { significantly }\end{array}$ & 4 \\
\hline Saleh et al., 2005 & Kuwait & 60 & 68 & & $24-56$ & $\begin{array}{l}\text { Prospective } \\
\quad \text { observational }^{*}\end{array}$ & $\begin{array}{l}\text { WC } \\
\text { HDL } \\
\text { TAG } \\
\text { FG }\end{array}$ & $\begin{array}{l}\text { Male: } 94.68 \mathrm{~cm} \\
\text { Female: } 89.76 \mathrm{~cm} \\
\text { Male: } 1.02 \mathrm{mmol} / / \\
\text { Female: } 1.27 \mathrm{mmol} / \mathrm{l} \\
\text { Male: } 1.33 \mathrm{mmol} / \mathrm{l} \\
\text { Female: } 1.38 \mathrm{mmol} / / \\
\text { Male: } 5.55 \mathrm{mmol} / \mathrm{l} \\
\text { Female: } 5.4 \mathrm{mmol} / /\end{array}$ & $\begin{array}{c}11.01 \\
17.52 \\
0.33 \\
0.34 \\
0.6 \\
0.96 \\
0.58 \\
0.89\end{array}$ & $\begin{array}{l}\text { Male: } 92 \mathrm{~cm} \\
\text { Female: } 87.18 \mathrm{~cm} \\
\text { Male: } 1.04 \mathrm{mmol} / \mathrm{l} \\
\text { Female: } 1.32 \mathrm{mmol} / / \\
\text { Male: } 1.54 \mathrm{mmol} / / \\
\text { Female: } 1.19 \mathrm{mmol} / / \\
\text { Male: } 5.55 \mathrm{mmol} / \mathrm{l} \\
\text { Female: } 5.2 \mathrm{mmol} / \mathrm{l}\end{array}$ & $\begin{array}{l}10.7 \\
17.53 \\
0.37 \\
0.36 \\
1.19 \\
0.8 \\
0.55 \\
0.54\end{array}$ & $\begin{array}{l}\text { WC significantly } \\
\text { decreased in males } \\
\text { and females }\end{array}$ & 4 \\
\hline Al-Numair, $2006^{(45)}$ & $\begin{array}{l}\text { Saudi } \\
\text { Arabia }\end{array}$ & 45 & 100 & & $30-45$ & $\begin{array}{l}\text { Prospective } \\
\quad \text { observational }^{*}\end{array}$ & $\begin{array}{l}\text { HDL } \\
\text { TAG } \\
\text { FG }\end{array}$ & $\begin{array}{l}1.79 \mathrm{mmol} / \mathrm{l} \\
1.48 \mathrm{mmol} / \mathrm{l} \\
4.91 \mathrm{mmol} / \mathrm{l}\end{array}$ & $\begin{array}{l}0.23 \\
0.55 \\
0.5\end{array}$ & $\begin{array}{l}1.82 \mathrm{mmol} / \mathrm{l} \\
1.2 \mathrm{mmol} / \mathrm{l} \\
4.51 \mathrm{mmol} / \mathrm{l}\end{array}$ & $\begin{array}{l}0.25 \\
0.51 \\
0.52\end{array}$ & $\begin{array}{l}\text { Significant decrease in } \\
\text { FG and TAG; No } \\
\text { significant change in } \\
\text { serum HDL }\end{array}$ & 4 \\
\hline $\begin{array}{l}\text { Dewanti et al., } \\
2006^{(46)}\end{array}$ & Indonesia & 37 & 100 & $\begin{array}{l}39 \\
\text { (sD 10) }\end{array}$ & $17-62$ & $\begin{array}{l}\text { Prospective } \\
\text { observational }^{*}\end{array}$ & SBP & $134 \mathrm{mmHg}$ & 21 & $124 \mathrm{mmHg}$ & 17 & $\begin{array}{l}\text { SBP decreased } \\
\text { significantly }\end{array}$ & 2 \\
\hline $\begin{array}{l}\text { Farshidfar et al., } \\
2006^{(47)}\end{array}$ & Iran & 21 & NR & NR & & Pre-experimental & $\begin{array}{l}\text { HDL } \\
\text { TAG } \\
\text { FG }\end{array}$ & $\begin{array}{l}39.59 \mathrm{mg} / \mathrm{dl} \\
65.37 \mathrm{mg} / \mathrm{dl} \\
74.4 \mathrm{mg} / \mathrm{dl}\end{array}$ & $\begin{array}{l}15 \cdot 67 \\
36 \cdot 76 \\
16 \cdot 97\end{array}$ & $\begin{array}{l}43 \cdot 28 \mathrm{mg} / \mathrm{dl} \\
68 \cdot 34 \mathrm{mg} / \mathrm{dl} \\
62.09 \mathrm{mg} / \mathrm{dl}\end{array}$ & $\begin{array}{r}12 \cdot 21 \\
19.85 \\
6.92\end{array}$ & $\begin{array}{l}\text { Significant decrease in } \\
\text { FG and significant } \\
\text { increase in HDL (on } \\
\text { day } 28 \text { of Ramadan) }\end{array}$ & 2 \\
\hline Lamine et al., 2006 ${ }^{(48)}$ & Tunisia & 30 & 30 & $\begin{array}{c}23 \cdot 7 \\
(\mathrm{sD} 2 \cdot 2)\end{array}$ & & $\begin{array}{l}\text { Prospective } \\
\quad \text { observational }^{*}\end{array}$ & $\begin{array}{l}\text { HDL } \\
\text { TAG } \\
\text { FG }\end{array}$ & $\begin{array}{l}1.1 \mathrm{mmol} / / \\
0.8 \mathrm{mmol} / \mathrm{l} \\
5.4 \mathrm{mmol} / \mathrm{l}\end{array}$ & $\begin{array}{l}0.4 \\
0.3 \\
0.6\end{array}$ & $\begin{array}{l}1.3 \mathrm{mmol} / \mathrm{l} \\
0.7 \mathrm{mmol} / \mathrm{l} \\
6.3 \mathrm{mmol} / \mathrm{l}\end{array}$ & $\begin{array}{l}0.4 \\
0.3 \\
0.6\end{array}$ & $\begin{array}{l}\text { Significant increase in } \\
\text { HDL }\end{array}$ & 4 \\
\hline Ziaee et al., 2006 ${ }^{(49)}$ & Iran & 81 & 51 & $\begin{array}{c}22 \cdot 7 \\
(\mathrm{SD} 2 \cdot 3)\end{array}$ & $20-35$ & Cohort & $\begin{array}{l}\text { HDL } \\
\text { TAG } \\
\text { FG }\end{array}$ & $\begin{array}{l}40 \mathrm{mg} / \mathrm{dl} \\
66.6 \mathrm{mg} / \mathrm{dl} \\
76.6 \mathrm{mg} / \mathrm{dl}\end{array}$ & $\begin{array}{r}9.9 \\
35.7 \\
7.5\end{array}$ & $\begin{array}{l}36.4 \mathrm{mg} / \mathrm{dl} \\
69.7 \mathrm{mg} / \mathrm{dl} \\
69.2 \mathrm{mg} / \mathrm{dl}\end{array}$ & $\begin{array}{l}8 \cdot 4 \\
4 \\
5 \cdot 7\end{array}$ & $\begin{array}{l}\text { FG and } \mathrm{HDL} \\
\text { decreased } \\
\text { significantly; No } \\
\text { significant change in } \\
\text { TAG }\end{array}$ & 4 \\
\hline $\begin{array}{l}\text { Aksungar et al., } \\
2007^{(50)}\end{array}$ & Turkey & 40 & 50 & & $20-39$ & Case-control & $\begin{array}{l}\mathrm{HDL} \\
\mathrm{TAG}\end{array}$ & $\begin{array}{l}\text { Male: } 88 \cdot 64 \mathrm{mg} / \mathrm{dl} \\
\text { Female: } 69.44 \mathrm{mg} / \mathrm{dl} \\
\text { Male: } 46.82 \mathrm{mg} / \mathrm{dl} \\
\text { Female: } 48.51 \mathrm{mg} / \mathrm{dl}\end{array}$ & $\begin{array}{r}44.49 \\
26.98 \\
7.69 \\
11.68\end{array}$ & $\begin{array}{l}\text { Male: } 91.64 \mathrm{mg} / \mathrm{dl} \\
\text { Female: } 64.88 \mathrm{mg} / \mathrm{dl} \\
\text { Male: } 50.67 \mathrm{mg} / \mathrm{dl} \\
\text { Female: } 56.46 \mathrm{mg} / \mathrm{dl}\end{array}$ & $\begin{array}{r}67.89 \\
35.13 \\
7.07 \\
8.07\end{array}$ & $\begin{array}{l}\text { HDL levels significantly } \\
\text { increased in females }\end{array}$ & 4 \\
\hline $\begin{array}{l}\text { Furuncuoglu et al., } \\
2007^{(51)}\end{array}$ & Turkey & 39 & 17.9 & $\begin{array}{l}28 \\
(\mathrm{SD} 8 \cdot 18)\end{array}$ & & $\begin{array}{l}\text { Prospective } \\
\quad \text { observational }^{*}\end{array}$ & $\begin{array}{l}\text { HDL } \\
\text { TAG } \\
\text { FG }\end{array}$ & $\begin{array}{l}45.7 \mathrm{mg} / \mathrm{dl} \\
110 \mathrm{mg} / \mathrm{dl} \\
83.9 \mathrm{mg} / \mathrm{dl}\end{array}$ & & $\begin{array}{l}43.9 \mathrm{mg} / \mathrm{dl} \\
94 \mathrm{mg} / \mathrm{dl} \\
73.6 \mathrm{mg} / \mathrm{dl}\end{array}$ & & $\begin{array}{l}\text { TAG and FG } \\
\text { decreased } \\
\text { significantly; HDL did } \\
\text { not change }\end{array}$ & 4 \\
\hline
\end{tabular}




\section{NS British Journal of Nutrition}

Table 1. Continued

\begin{tabular}{|c|c|c|c|c|c|c|c|c|c|c|c|c|c|}
\hline \multirow{2}{*}{$\begin{array}{l}\text { Study, publication } \\
\text { year }\end{array}$} & \multirow[b]{2}{*}{ Country } & \multicolumn{2}{|c|}{ Sample size } & \multicolumn{2}{|c|}{ Age (years) } & \multirow[b]{2}{*}{ Study design } & \multirow{2}{*}{$\begin{array}{c}\text { Examined } \\
\text { markers }\end{array}$} & \multicolumn{2}{|c|}{ Before Ramadan $\dagger$} & \multicolumn{2}{|c|}{ End of Ramadan $†$} & \multirow{2}{*}{$\begin{array}{l}\text { Results (after } \\
\text { Ramadan month } \\
\text { compared with before) }\end{array}$} & \multirow{2}{*}{$\begin{array}{l}\text { Total } \\
\text { quality } \\
\text { score }\end{array}$} \\
\hline & & $n$ & $\%$ Male & Mean & Range & & & Mean & SD & Mean & SD & & \\
\hline Mansi, 2007(52) & Jordan & 70 & NR & $\begin{array}{l}21 \\
(\text { SD } 1 \cdot 6)\end{array}$ & & Cohort & $\begin{array}{l}\text { HDL } \\
\text { TAG } \\
\text { FG } \\
\text { SBP }\end{array}$ & $\begin{array}{l}36.13 \mathrm{mg} / \mathrm{dl} \\
148.54 \mathrm{mg} / \mathrm{dl} \\
94.32 \mathrm{mg} / \mathrm{dl} \\
126.32 \mathrm{mmHg}\end{array}$ & $\begin{array}{r}6 \cdot 42 \\
54.72 \\
6 \cdot 23 \\
17 \cdot 46\end{array}$ & $\begin{array}{l}48.86 \mathrm{mg} / \mathrm{dl} \\
139.36 \mathrm{mg} / \mathrm{dl} \\
85.84 \mathrm{mg} / \mathrm{dl} \\
112.4 \mathrm{mmHg}\end{array}$ & $\begin{array}{r}12 \cdot 34 \\
52 \cdot 29 \\
6 \cdot 43 \\
15\end{array}$ & $\begin{array}{l}\text { SBP significantly } \\
\text { decreased; HDL } \\
\text { significantly } \\
\text { increased }\end{array}$ & 2 \\
\hline $\begin{array}{l}\text { Mansi \& Amneh, } \\
\text { 2007(53) }\end{array}$ & Jordan & 42 & 100 & $\begin{array}{l}21 \cdot 3 \\
(\mathrm{SD} 1 \cdot 6)\end{array}$ & & $\begin{array}{l}\text { Prospective } \\
\quad \text { observational }^{*}\end{array}$ & $\begin{array}{l}\text { HDL } \\
\text { TAG } \\
\text { FG } \\
\text { SBP }\end{array}$ & $\begin{array}{l}36.13 \mathrm{mg} / \mathrm{dl} \\
148.54 \mathrm{mg} / \mathrm{dl} \\
88.4 \mathrm{mg} / \mathrm{dl} \\
126.32 \mathrm{mmHg}\end{array}$ & $\begin{array}{c}6 \cdot 42 \\
54 \cdot 72 \\
9 \\
17 \cdot 46\end{array}$ & $\begin{array}{l}48.86 \mathrm{mg} / \mathrm{dl} \\
139.36 \mathrm{mg} / \mathrm{dl} \\
62.9 \mathrm{mg} / \mathrm{dl} \\
112.41 \mathrm{mmHg}\end{array}$ & $\begin{array}{l}12 \cdot 34 \\
52 \cdot 29 \\
7 \cdot 7 \\
15\end{array}$ & $\begin{array}{l}\text { SBP significantly } \\
\text { decreased; HDL } \\
\text { significantly } \\
\text { increased }\end{array}$ & 4 \\
\hline $\begin{array}{l}\text { Salehi \& Neghab, } \\
2007^{(54)}\end{array}$ & Iran & 28 & 100 & 23.4 & $20-26$ & $\begin{array}{l}\text { Prospective } \\
\text { observational }^{*}\end{array}$ & $\begin{array}{l}\text { TAG } \\
\text { FG }\end{array}$ & $\begin{array}{l}195 \mathrm{mg} / \mathrm{dl} \\
81 \mathrm{mg} / \mathrm{dl}\end{array}$ & $\begin{array}{l}31 \\
23\end{array}$ & $\begin{array}{l}197 \mathrm{mg} / \mathrm{dl} \\
69 \mathrm{mg} / \mathrm{dl}\end{array}$ & $\begin{array}{r}19 \\
8\end{array}$ & $\begin{array}{l}\text { Mean FG significantly } \\
\text { decreased }\end{array}$ & 4 \\
\hline $\begin{array}{l}\text { Shariatpanahi et al., } \\
2008^{(55)}\end{array}$ & Iran & 55 & 100 & $\begin{array}{l}34 \cdot 1 \\
(\mathrm{sD} 8 \cdot 9)\end{array}$ & $34-61$ & $\begin{array}{l}\text { Prospective } \\
\quad \text { observational }^{\star}\end{array}$ & $\begin{array}{l}\text { WC } \\
\text { HDL } \\
\text { TAG } \\
\text { FG } \\
\text { SBP }\end{array}$ & $\begin{array}{l}94.81 \mathrm{~cm} \\
42.87 \mathrm{mg} / \mathrm{dl} \\
210 \mathrm{mg} / \mathrm{dl} \\
89.45 \mathrm{mg} / \mathrm{dl} \\
115 \mathrm{mmHg}\end{array}$ & $\begin{array}{c}7.8 \\
5.45 \\
139.6 \\
28.79 \\
13.57\end{array}$ & $\begin{array}{l}91.98 \mathrm{~cm} \\
46.24 \mathrm{mg} / \mathrm{dl} \\
232.78 \mathrm{mg} / \mathrm{dl} \\
81.21 \mathrm{mg} / \mathrm{dl} \\
108.93 \mathrm{mmHg}\end{array}$ & $\begin{array}{r}7.7 \\
5.5 \\
108 \cdot 87 \\
17.84 \\
11.57\end{array}$ & $\begin{array}{l}\text { HDL significantly } \\
\text { increased; FG, WC } \\
\text { and SBP } \\
\text { significantly } \\
\text { decreased }\end{array}$ & 4 \\
\hline Ibrahim et al., 2008 ${ }^{(56)}$ & UAE & 14 & 64 & & $25-58$ & $\begin{array}{l}\text { Prospective } \\
\text { observational }^{*}\end{array}$ & $\begin{array}{l}\text { TAG } \\
\text { FG }\end{array}$ & $\begin{array}{l}116.9 \mathrm{mg} / \mathrm{dl} \\
109.3 \mathrm{mg} / \mathrm{dl}\end{array}$ & $\begin{array}{r}35.4 \\
6.6\end{array}$ & $\begin{array}{l}87.5 \mathrm{mg} / \mathrm{dl} \\
96.4 \mathrm{mg} / \mathrm{dl}\end{array}$ & $\begin{array}{l}23.4 \\
11.4\end{array}$ & $\begin{array}{l}\mathrm{FG} \text { and TAG } \\
\text { significantly } \\
\text { decreased }\end{array}$ & 4 \\
\hline $\begin{array}{l}\text { Al Hourani et al., } \\
2009^{(57)}\end{array}$ & Jordan & 57 & 0 & $\begin{array}{l}21.6 \\
(\mathrm{SD} 4 \cdot 14)\end{array}$ & $18-29$ & $\begin{array}{l}\text { Prospective } \\
\text { observational }^{*}\end{array}$ & $\begin{array}{l}\text { HDL } \\
\text { TAG }\end{array}$ & $\begin{array}{l}59.3 \mathrm{mg} / \mathrm{dl} \\
88.3 \mathrm{mg} / \mathrm{dl}\end{array}$ & $\begin{array}{r}9.5 \\
62.5\end{array}$ & $\begin{array}{l}62.3 \mathrm{mg} / \mathrm{dl} \\
65.4 \mathrm{mg} / \mathrm{dl}\end{array}$ & $\begin{array}{l}14 \cdot 6 \\
20 \cdot 8\end{array}$ & $\begin{array}{l}\text { No significant change } \\
\text { in HDL or TAG }\end{array}$ & 4 \\
\hline $\begin{array}{l}\text { Lamri-Senhadji et al., } \\
2009^{(58)}\end{array}$ & Algeria & 46 & 48 & $\begin{array}{l}24 \\
(\mathrm{SD} 3)\end{array}$ & & Prospective & $\begin{array}{l}\mathrm{HDL} \\
\mathrm{TAG}\end{array}$ & $\begin{array}{l}\text { Male: } 1.7 \mathrm{~g} / \mathrm{l} \\
\text { Female: } 2 \mathrm{~g} / \mathrm{l} \\
\text { Male: } 0.72 \mathrm{~g} / \mathrm{l} \\
\text { Female: } 0.67 \mathrm{~g} / \mathrm{l}\end{array}$ & $\begin{array}{l}0.26 \\
0.42 \\
0.36 \\
0.26\end{array}$ & $\begin{array}{l}\text { Male: } 2.22 \mathrm{~g} / \mathrm{l} \\
\text { Female: } 2.7 \mathrm{~g} / \mathrm{l} \\
\text { Male: } 0.69 \mathrm{~g} / \mathrm{l} \\
\text { Female: } 0.93 \mathrm{~g} / \mathrm{l}\end{array}$ & $\begin{array}{l}0.3 \\
0.2 \\
0.36 \\
0.5\end{array}$ & $\begin{array}{l}\text { HDL was } 1.4 \text {-fold } \\
\text { higher in males and } \\
\text { females }\end{array}$ & 4 \\
\hline Sülü et al., 2010(59) & Turkey & 45 & $51 \cdot 1$ & $28 \cdot 7$ & $21-25$ & $\begin{array}{l}\text { Prospective } \\
\text { observational }^{*}\end{array}$ & $\begin{array}{l}\text { HDL } \\
\text { TAG } \\
\text { FG }\end{array}$ & $\begin{array}{l}45 \mathrm{mg} / \mathrm{dl} \\
142.9 \mathrm{mg} / \mathrm{dl} \\
85.6 \mathrm{mg} / \mathrm{dl}\end{array}$ & $\begin{array}{r}11 \cdot 2 \\
61 \cdot 1 \\
7 \cdot 2\end{array}$ & $\begin{array}{l}49.0 \mathrm{mg} / \mathrm{dl} \\
105.8 \mathrm{mg} / \mathrm{dl} \\
92.8 \mathrm{mg} / \mathrm{dl}\end{array}$ & $\begin{array}{r}10 \cdot 9 \\
57 \cdot 1 \\
7 \cdot 1\end{array}$ & $\begin{array}{l}\text { Significant increase in } \\
\text { FG and HDL; } \\
\text { Significant decrease } \\
\text { in TAG }\end{array}$ & 4 \\
\hline $\begin{array}{l}\text { Norouzy et al., } \\
2010^{(60)}\end{array}$ & Iran & 240 & 66 & 40 & $18-70$ & $\begin{array}{l}\text { Prospective } \\
\text { cohort }\end{array}$ & WC & $92.07 \mathrm{~cm}$ & $11 \cdot 1$ & $90 \cdot 71 \mathrm{~cm}$ & $10 \cdot 94$ & $\begin{array}{l}\text { Significant reduction in } \\
\text { WC }\end{array}$ & 4 \\
\hline Barkia et al., 2011(61) & Tunisia & 25 & 76 & 42 & $22-55$ & $\begin{array}{l}\text { Prospective } \\
\text { observational }^{*}\end{array}$ & $\begin{array}{l}\text { HDL } \\
\text { TAG } \\
\text { FG }\end{array}$ & $\begin{array}{l}1.0 \mathrm{mmol} / / \\
1.1 \mathrm{mmol} / / \\
4.7 \mathrm{mmol} / \mathrm{l}\end{array}$ & $\begin{array}{l}0.2 \\
0.5 \\
0.8\end{array}$ & $\begin{array}{l}1.0 \mathrm{mmol} / / \\
1.1 \mathrm{mmol} / \mathrm{l} \\
4.9 \mathrm{mmol} / \mathrm{l}\end{array}$ & $\begin{array}{l}0.3 \\
0.3 \\
0.9\end{array}$ & $\begin{array}{l}\text { No significant change } \\
\text { in WC, TAG, HDL, } \\
\text { SBP or FG }\end{array}$ & 4 \\
\hline Mohammed, 2011(62) & Iraq & 56 & 100 & $\begin{array}{c}48 \cdot 4 \\
\text { (sD } 7 \cdot 15)\end{array}$ & & Prospective & $\begin{array}{l}\text { HDL } \\
\text { TAG } \\
\text { FG }\end{array}$ & $\begin{array}{l}0.8 \mathrm{mmol} / \mathrm{l} \\
1.6 \mathrm{mmol} / \mathrm{l} \\
5.3 \mathrm{mmol} / \mathrm{l}\end{array}$ & $\begin{array}{l}0.2 \\
0.4 \\
0.15\end{array}$ & $\begin{array}{l}0.9 \mathrm{mmol} / \mathrm{l} \\
0.9 \mathrm{mmol} / \mathrm{l} \\
4.2 \mathrm{mmol} / \mathrm{l}\end{array}$ & $\begin{array}{l}0.7 \\
0.7 \\
0.3\end{array}$ & $\begin{array}{l}\text { Significant increase in } \\
\text { HDL and decrease } \\
\text { in TAG }\end{array}$ & 4 \\
\hline $\begin{array}{l}\text { Ünalacak et al., } \\
2011^{(63)}\end{array}$ & Turkey & 20 & 100 & $\begin{array}{l}27 \cdot 4 \\
(\mathrm{SD} 5 \cdot 2)\end{array}$ & & Cross-sectional & $\begin{array}{l}\text { HDL } \\
\text { TAG } \\
\text { FG } \\
\text { SBP }\end{array}$ & $\begin{array}{l}\text { Obese: } 43 \mathrm{mg} / \mathrm{dl} \\
\text { Normal: } 45 \mathrm{mg} / \mathrm{dl} \\
\text { Obese: } 151 \mathrm{mg} / \mathrm{dl} \\
\text { Normal: } 120 \mathrm{mg} / \mathrm{dl} \\
\text { Obese: } 97.2 \mathrm{mg} / \mathrm{dl} \\
\text { Normal: } 90.5 \mathrm{mg} / \mathrm{dl} \\
\text { Obese: } 120 \mathrm{mmHg} \\
\text { Normal: } 118 \mathrm{mmHg}\end{array}$ & $\begin{array}{c}10 \\
5 \\
41 \\
59 \\
13 \cdot 5 \\
6 \cdot 6 \\
8 \\
8\end{array}$ & $\begin{array}{l}\text { Obese: } 43 \mathrm{mg} / \mathrm{dl} \\
\text { Normal: } 45 \mathrm{mg} / \mathrm{dl} \\
\text { Obese: } 129 \mathrm{mg} / \mathrm{dl} \\
\text { Normal: } 93 \mathrm{mg} / \mathrm{dl} \\
\text { Obese: } 93 \mathrm{mg} / \mathrm{dl} \\
\text { Normal: } 89 \cdot 2 \mathrm{mg} / \mathrm{dl} \\
\text { Obese: } 114 \mathrm{mmHg} \\
\text { Normal: } 112 \mathrm{mmHg}\end{array}$ & $\begin{array}{r}6 \\
4 \\
39 \\
53 \\
7 \\
5 \\
8 \\
8\end{array}$ & $\begin{array}{l}\text { Significant decrease in } \\
\text { FG in obese group; } \\
\text { SBP and TAG } \\
\text { significantly reduced } \\
\text { in obese and non- } \\
\text { obese groups }\end{array}$ & 4 \\
\hline Faris et al., 2012(18) & Jordan & 50 & 42 & $\begin{array}{l}32.7 \\
(\mathrm{SD} 9.5)\end{array}$ & $18-51$ & Cross-sectional & $\begin{array}{l}\text { WC } \\
\text { SBP }\end{array}$ & $\begin{array}{l}83.62 \mathrm{~cm} \\
112.3 \mathrm{mmHg}\end{array}$ & $\begin{array}{l}11 \cdot 17 \\
10 \cdot 01\end{array}$ & $\begin{array}{l}82.69 \mathrm{~cm} \\
104.4 \mathrm{mmHg}\end{array}$ & $\begin{array}{r}10 \cdot 34 \\
9 \cdot 07\end{array}$ & $\begin{array}{l}\text { SBP significantly } \\
\text { decreased }\end{array}$ & 4 \\
\hline
\end{tabular}




\section{NS British Journal of Nutrition}

Table 1. Continued

\begin{tabular}{|c|c|c|c|c|c|c|c|c|c|c|c|c|c|}
\hline \multirow{2}{*}{$\begin{array}{l}\text { Study, publication } \\
\text { year }\end{array}$} & \multirow[b]{2}{*}{ Country } & \multicolumn{2}{|c|}{ Sample size } & \multicolumn{2}{|c|}{ Age (years) } & \multirow[b]{2}{*}{ Study design } & \multirow{2}{*}{$\begin{array}{l}\text { Examined } \\
\text { markers }\end{array}$} & \multicolumn{2}{|c|}{ Before Ramadan† } & \multicolumn{2}{|c|}{ End of Ramadan† } & \multirow{2}{*}{$\begin{array}{l}\text { Results (after } \\
\text { Ramadan month } \\
\text { compared with before) }\end{array}$} & \multirow{2}{*}{$\begin{array}{l}\text { Total } \\
\text { quality } \\
\text { score }\end{array}$} \\
\hline & & $n$ & $\%$ Male & Mean & Range & & & Mean & SD & Mean & SD & & \\
\hline \multirow[t]{8}{*}{ Shehab et al., 2012 (64) } & \multirow[t]{8}{*}{ UAE } & \multirow[t]{8}{*}{60} & \multirow[t]{8}{*}{65} & \multirow[t]{8}{*}{$\begin{array}{c}43.2 \\
\text { (sD } 9.4)\end{array}$} & & \multirow[t]{8}{*}{$\begin{array}{l}\text { Prospective } \\
\text { observational }^{\star}\end{array}$} & \multirow[t]{2}{*}{ WC } & Male: $96.9 \mathrm{~cm}$ & $10 \cdot 9$ & Male: $94.5 \mathrm{~cm}$ & $11 \cdot 1$ & \multirow{8}{*}{$\begin{array}{l}\text { Significant and } \\
\text { beneficial change in } \\
\text { SBP, WC, TAG and } \\
\text { HDL }\end{array}$} & \multirow[t]{8}{*}{4} \\
\hline & & & & & & & & Female: $79.6 \mathrm{~cm}$ & 14.9 & Female: $77.2 \mathrm{~cm}$ & $15 \cdot 1$ & & \\
\hline & & & & & & & \multirow[t]{2}{*}{$\mathrm{HDL}$} & Male: $0.8 \mathrm{mmol} / \mathrm{l}$ & 0.2 & Male: $0.8 \mathrm{mmol} / \mathrm{l}$ & 0.3 & & \\
\hline & & & & & & & & Female: $0.9 \mathrm{mmol} / \mathrm{l}$ & 0.4 & Female: $0.9 \mathrm{mmol} / \mathrm{l}$ & 0.4 & & \\
\hline & & & & & & & \multirow[t]{2}{*}{ TAG } & Male: $1.2 \mathrm{mmol} / \mathrm{l}$ & 1 & Male: $1.1 \mathrm{mmol} / \mathrm{l}$ & 1 & & \\
\hline & & & & & & & & Female: $0.7 \mathrm{mmol} / \mathrm{l}$ & 0.5 & Female: $1.2 \mathrm{mmol} / \mathrm{l}$ & 1.2 & & \\
\hline & & & & & & & \multirow[t]{2}{*}{ SBP } & Male: $124.1 \mathrm{mmHg}$ & $14 \cdot 6$ & Male: $120.8 \mathrm{mmHg}$ & 13.8 & & \\
\hline & & & & & & & & $\begin{array}{l}\text { Female: } \\
\quad 117.6 \mathrm{mmHg}\end{array}$ & $12 \cdot 3$ & Female: $113.5 \mathrm{mmHg}$ & $11 \cdot 2$ & & \\
\hline $\begin{array}{l}\text { Sayedda et al., } \\
2013^{(65)}\end{array}$ & India & 20 & 100 & $\begin{array}{c}24.65 \\
\text { (SD } 4.4)\end{array}$ & $19-32$ & $\begin{array}{l}\text { Prospective } \\
\text { observational }^{*}\end{array}$ & WC & $84.25 \mathrm{~cm}$ & $5 \cdot 44$ & $82 \cdot 15 \mathrm{~cm}$ & $6 \cdot 09$ & $\begin{array}{l}\text { WC significantly } \\
\text { decreased }\end{array}$ & 4 \\
\hline Agoumi et al., 2013 & Spain & 55 & 40 & & $18-70$ & Cohort & WC & $101.63 \mathrm{~cm}$ & $12 \cdot 0$ & $99.36 \mathrm{~cm}$ & 11.24 & WC decreased & 4 \\
\hline \multirow[t]{3}{*}{ Bahijri et al., 2013 (67) } & \multirow{3}{*}{$\begin{array}{l}\text { Saudi } \\
\text { Arabia }\end{array}$} & 23 & 78 & 23.1 & & Prospective & $\mathrm{HDL}$ & $1.2 \mathrm{mmol} / \mathrm{l}$ & 0.05 & $1.1 \mathrm{mmol} / \mathrm{l}$ & 0.03 & Statistically significant & 4 \\
\hline & & & & (sD 1.2) & & observational* & TAG & $0.85 \mathrm{mmol} / \mathrm{l}$ & 0.12 & $121 \mathrm{mmol} / \mathrm{l}$ & 0.11 & decrease in $\mathrm{HDL}$ & \\
\hline & & & & & & & $\mathrm{FG}$ & $5.33 \mathrm{mmol} / \mathrm{l}$ & 0.07 & $5.62 \mathrm{mmol} / \mathrm{l}$ & 0.11 & & \\
\hline Haouari-Oukerro & Tunisia & 38 & 100 & 20.8 & $18-23$ & Prospective & $\mathrm{HDL}$ & $1.16 \mathrm{mmol} / \mathrm{l}$ & 0.05 & $1.39 \mathrm{mmol} / \mathrm{l}$ & 0.08 & Significant increase in & 4 \\
\hline et al., 2013 ${ }^{(68)}$ & & & & (sD 1) & & observational* & TAG & $0.97 \mathrm{mmol} / \mathrm{l}$ & 0.03 & $0.78 \mathrm{mmol} / \mathrm{l}$ & 0.03 & HDL; significant & \\
\hline & & & & & & & $F G$ & $4.94 \mathrm{mmol} / \mathrm{l}$ & 0.11 & $4.55 \mathrm{mmol} / \mathrm{l}$ & 0.39 & decrease in $F G$ & \\
\hline $\begin{array}{l}\text { Hosseini et al., } \\
2013^{(69)}\end{array}$ & Iran & 11 & 0 & & $20-45$ & $\begin{array}{l}\text { Semi- } \\
\quad \text { experimental }\end{array}$ & $\mathrm{FG}$ & $87 \cdot 2 \mathrm{mg} / \mathrm{dl}$ & $5 \cdot 1$ & $83.3 \mathrm{mg} / \mathrm{dl}$ & $7 \cdot 9$ & $\begin{array}{l}\text { No significant change } \\
\text { in FG }\end{array}$ & 4 \\
\hline Akrami et al., $2013^{(70)}$ & Iran & 58 & NR & & $20-40$ & Prospective & $\mathrm{HDL}$ & $52.1 \mathrm{mg} / \mathrm{dl}$ & 4.64 & $50.85 \mathrm{mg} / \mathrm{dl}$ & 5.96 & Significant difference in & 2 \\
\hline & & & & & & observational $^{*}$ & TAG & $151.55 \mathrm{mg} / \mathrm{dl}$ & 94.6 & $125.6 \mathrm{mg} / \mathrm{dl}$ & 64.8 & FG and TAG levels & \\
\hline & & & & & & & $\mathrm{FG}$ & $122.25 \mathrm{mg} / \mathrm{dl}$ & 55 & $110.75 \mathrm{mg} / \mathrm{dl}$ & 40.04 & & \\
\hline $\begin{array}{l}\text { Norouzy et al., } \\
2013^{(71)}\end{array}$ & Iran & 240 & 66 & $\begin{array}{c}40.1 \\
(\mathrm{SD} 0.7)\end{array}$ & $18-70$ & $\begin{array}{l}\text { Prospective } \\
\text { observational }\end{array}$ & WC & $\begin{array}{l}<35 \text { years males: } \\
92.9 \mathrm{~cm}\end{array}$ & $1 \cdot 1$ & $\begin{array}{l}<35 \text { years males: } \\
91.6 \mathrm{~cm}\end{array}$ & $1 \cdot 2$ & $\begin{array}{l}\text { WC decreased } \\
\text { significantly in most }\end{array}$ & 4 \\
\hline & & & & & & & & $\begin{array}{l}35-70 \text { years males: } \\
96.4 \mathrm{~cm}\end{array}$ & 0.8 & $\begin{array}{l}35-70 \text { years males: } \\
94.6 \mathrm{~cm}\end{array}$ & 0.8 & & \\
\hline & & & & & & & & $\begin{array}{l}<35 \text { years females: } \\
81.6 \mathrm{~cm}\end{array}$ & 1.6 & $\begin{array}{l}<35 \text { years females: } \\
80.6 \mathrm{~cm}\end{array}$ & 1.5 & years & \\
\hline & & & & & & & & $\begin{array}{l}\text { 35-70 years } \\
\text { females: } 90.5 \mathrm{~cm}\end{array}$ & 1.8 & $\begin{array}{l}35-70 \text { years } \\
\text { females: } 90.5 \mathrm{~cm}\end{array}$ & 1.9 & & \\
\hline Pirsaheb et al., & Iran & 152 & 100 & 39.4 & $21-63$ & Interventional & $\mathrm{HDL}$ & $44.7 \mathrm{mg} / \mathrm{dl}$ & 7.9 & $45.59 \mathrm{mg} / \mathrm{dl}$ & 9 & TAG levels decreased & 4 \\
\hline $2013^{(72)}$ & & & & (SD 10.7) & & cohort & TAG & $151.44 \mathrm{mg} / \mathrm{dl}$ & $85 \cdot 2$ & $140.44 \mathrm{mg} / \mathrm{dl}$ & $75 \cdot 2$ & significantly; SBP & \\
\hline & & & & & & & $\mathrm{FG}$ & $126.96 \mathrm{mmHg}$ & 14.6 & $123.93 \mathrm{mmHg}$ & $15 \cdot 2$ & $\begin{array}{l}\text { decreased } \\
\text { significantly }\end{array}$ & \\
\hline Rohin et al., 2013(73) & Malaysia & 46 & 30 & 33.04 & $25-40$ & Prospective & WC & Normal: $72.23 \mathrm{~cm}$ & $6 \cdot 39$ & Normal: $70.05 \mathrm{~cm}$ & $5 \cdot 67$ & WC significantly & 4 \\
\hline & & & & (SD 4.6) & & observational* ${ }^{*}$ & & $\begin{array}{l}\text { Overweight: } \\
82.13 \mathrm{~cm}\end{array}$ & $6 \cdot 74$ & $\begin{array}{l}\text { Overweight: } \\
83.44 \mathrm{~cm}\end{array}$ & 2.03 & $\begin{array}{l}\text { decreased in normal } \\
\text { weight group }\end{array}$ & \\
\hline & & & & & & & & Obese: $91.91 \mathrm{~cm}$ & $6 \cdot 37$ & Obese: $90.66 \mathrm{~cm}$ & 7.85 & & \\
\hline Rabiee et al., 2014(74) & Iran & 49 & 0 & & $20-45$ & Cohort & $\mathrm{HDL}$ & $\begin{array}{l}\text { Consuming downset } \\
\text { meal: } 49.35 \mathrm{mg} / \mathrm{dl}\end{array}$ & $7 \cdot 4$ & $\begin{array}{l}\text { Consuming downset } \\
\text { meal: } 48.9 \mathrm{mg} / \mathrm{dl}\end{array}$ & $12 \cdot 15$ & $\begin{array}{l}\text { Significant increase in } \\
\text { TAG and significant }\end{array}$ & 4 \\
\hline & & & & & & & & $\begin{array}{l}\text { Non-consuming } \\
\text { downset meal: } \\
49.85 \mathrm{mg} / \mathrm{dl}\end{array}$ & $11 \cdot 25$ & $\begin{array}{l}\text { Non-consuming } \\
\text { downset meal: } \\
49.1 \mathrm{mg} / \mathrm{dl}\end{array}$ & $9 \cdot 6$ & $\begin{array}{l}\text { decrease in HDL in } \\
\text { fasting individuals }\end{array}$ & \\
\hline & & & & & & & TAG & $\begin{array}{l}\text { Consuming downset } \\
\text { meal: } 96.9 \mathrm{mg} / \mathrm{dl}\end{array}$ & 49.7 & $\begin{array}{l}\text { Consuming downset } \\
\text { meal: } 112.45 \mathrm{mg} / \mathrm{dl}\end{array}$ & 65.65 & & \\
\hline & & & & & & & & $\begin{array}{l}\text { Non-consuming } \\
\text { downset meal: } \\
111.85 \mathrm{mg} / \mathrm{d}\end{array}$ & 61.55 & $\begin{array}{l}\text { Non-consuming } \\
\text { downset meal: } \\
138.15 \mathrm{mg} / \mathrm{dl}\end{array}$ & $106 \cdot 75$ & & \\
\hline
\end{tabular}


N British Journal of Nutrition

Table 1. Continued

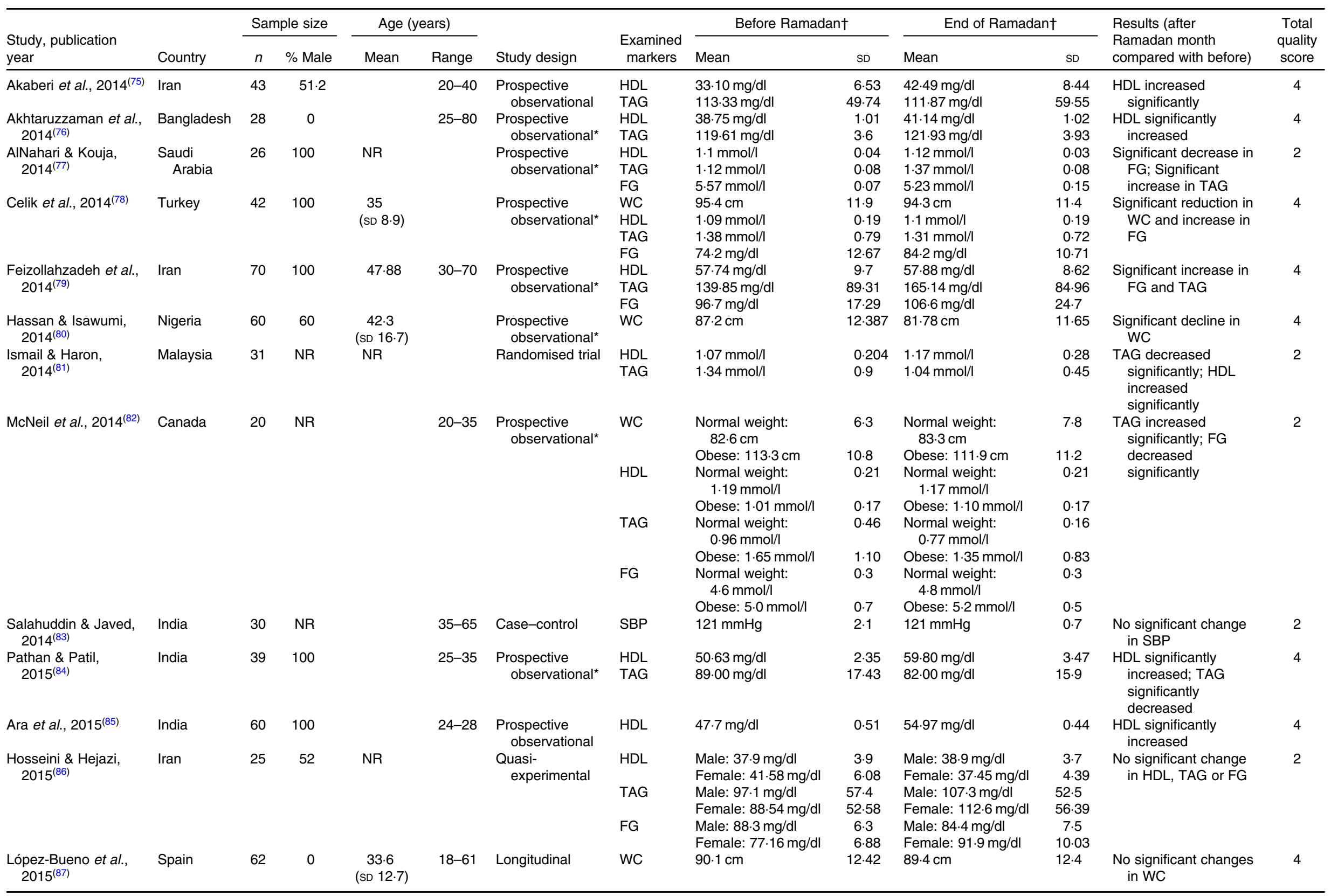




\section{N British Journal of Nutrition}

Table 1. Continued

\begin{tabular}{|c|c|c|c|c|c|c|c|c|c|c|c|c|c|}
\hline \multirow{2}{*}{$\begin{array}{l}\text { Study, publication } \\
\text { year }\end{array}$} & \multirow[b]{2}{*}{ Country } & \multicolumn{2}{|c|}{ Sample size } & \multicolumn{2}{|c|}{ Age (years) } & \multirow[b]{2}{*}{ Study design } & \multirow{2}{*}{$\begin{array}{l}\text { Examined } \\
\text { markers }\end{array}$} & \multicolumn{2}{|c|}{ Before Ramadan $\dagger$} & \multicolumn{2}{|c|}{ End of Ramadan $\dagger$} & \multirow{2}{*}{$\begin{array}{l}\text { Results (after } \\
\text { Ramadan month } \\
\text { compared with before) }\end{array}$} & \multirow{2}{*}{$\begin{array}{l}\text { Total } \\
\text { quality } \\
\text { score }\end{array}$} \\
\hline & & $n$ & $\%$ Male & Mean & Range & & & Mean & SD & Mean & SD & & \\
\hline $\begin{array}{l}\text { Shahsavan et al., } \\
2015^{(88)}\end{array}$ & Iran & 89 & 57 & 34.97 & $20-50$ & $\begin{array}{l}\text { Prospective } \\
\quad \text { observational }^{\star}\end{array}$ & $\begin{array}{l}\text { HDL } \\
\text { TAG }\end{array}$ & $\begin{array}{l}42.76 \mathrm{mg} / \mathrm{dl} \\
123.07 \mathrm{mg} / \mathrm{dl}\end{array}$ & 7.94 & $\begin{array}{l}41.53 \mathrm{mg} / \mathrm{dl} \\
133.96 \mathrm{mg} / \mathrm{dl}\end{array}$ & 6.98 & $\begin{array}{l}\text { Substantial decline in } \\
\text { HDL }\end{array}$ & 4 \\
\hline & & & & & & & SPB & $116.4 \mathrm{mmHg}$ & 11 & $115.5 \mathrm{mmHg}$ & $10 \cdot 1$ & & \\
\hline Suriani et al., $2015^{(89)}$ & Malaysia & 84 & 0 & $\begin{array}{c}39.8 \\
(\mathrm{SD} 10 \cdot 3)\end{array}$ & & $\begin{array}{l}\text { Prospective } \\
\quad \text { observational }^{\star}\end{array}$ & $\begin{array}{l}\text { HDL } \\
\text { TAG } \\
\text { FG }\end{array}$ & $\begin{array}{l}49.03 \mathrm{mg} / \mathrm{dl} \\
0.99 \mathrm{mmol} / \mathrm{l} \\
4.42 \mathrm{mmol} / \mathrm{l}\end{array}$ & $\begin{array}{c}13 \cdot 9 \\
0.4 \\
0.87\end{array}$ & $\begin{array}{l}34.36 \mathrm{mg} / \mathrm{dl} \\
0.89 \mathrm{mmol} / \mathrm{l} \\
4.24 \mathrm{mmol} / \mathrm{l}\end{array}$ & $\begin{array}{r}11.97 \\
0.31 \\
0.79\end{array}$ & $\begin{array}{l}\text { HDL, TAG and FG } \\
\text { significantly reduced }\end{array}$ & 4 \\
\hline & & & & & & & SBP & $124.51 \mathrm{mmHg}$ & $18 \cdot 14$ & $123.27 \mathrm{mmHg}$ & $16 \cdot 14$ & & \\
\hline Babaei et al., 2016(90) & Iran & 36 & 100 & $\begin{array}{c}39.11 \\
(\mathrm{SD} 8.6)\end{array}$ & & $\begin{array}{l}\text { Quasi- } \\
\quad \text { experimental }\end{array}$ & $\begin{array}{l}\text { HDL } \\
\text { TAG } \\
\text { FG }\end{array}$ & $\begin{array}{l}51.78 \mathrm{mg} / \mathrm{dl} \\
162.72 \mathrm{mg} / \mathrm{dl} \\
75.78 \mathrm{mg} / \mathrm{dl}\end{array}$ & $\begin{array}{r}12 \cdot 27 \\
94 \cdot 23 \\
8 \cdot 46\end{array}$ & $\begin{array}{l}49.81 \mathrm{mg} / \mathrm{dl} \\
144.22 \mathrm{mg} / \mathrm{dl} \\
80.06 \mathrm{mg} / \mathrm{dl}\end{array}$ & $\begin{array}{r}12 \cdot 14 \\
67 \cdot 06 \\
9.26\end{array}$ & $\begin{array}{l}\text { Significant reduction in } \\
\text { mean } F G \text { and TAG }\end{array}$ & 4 \\
\hline $\begin{array}{l}\text { BaHammam et al., } \\
2016^{(91)}\end{array}$ & $\begin{array}{l}\text { Saudi } \\
\text { Arabia }\end{array}$ & 80 & 100 & $\begin{array}{l}26.6 \\
(\mathrm{SD} 4.9)\end{array}$ & 20-35 & $\begin{array}{l}\text { Prospective } \\
\text { observational }^{\star}\end{array}$ & $F G$ & $5.7 \mathrm{mmol} / \mathrm{l}$ & 0.4 & $5.8 \mathrm{mmol} / \mathrm{l}$ & 0.5 & $\begin{array}{l}\text { No significant changes } \\
\text { in FG }\end{array}$ & 4 \\
\hline $\begin{array}{l}\text { Esmaeilzadeh \& } \\
\text { Borne, 2016 }\end{array}$ & Belgium & 14 & 100 & $\begin{array}{c}42.4 \\
(\mathrm{SD} 1.5)\end{array}$ & & $\begin{array}{l}\text { Prospective } \\
\text { case-control }\end{array}$ & $\begin{array}{l}\text { HDL } \\
\text { TAG } \\
\text { FG } \\
\text { SBP }\end{array}$ & $\begin{array}{l}51.8 \mathrm{mg} / \mathrm{dl} \\
106 \cdot 2 \mathrm{mg} / \mathrm{dl} \\
85 \cdot 6 \mathrm{mg} / \mathrm{dl} \\
117 \mathrm{mmHg}\end{array}$ & $\begin{array}{c}4 \cdot 2 \\
20 \\
1 \cdot 3 \\
3\end{array}$ & $\begin{array}{l}47.5 \mathrm{mg} / \mathrm{dl} \\
119.6 \mathrm{mg} / \mathrm{dl} \\
93.4 \mathrm{mg} / \mathrm{dl} \\
104.3 \mathrm{mmHg}\end{array}$ & $\begin{array}{r}3 \cdot 2 \\
30 \cdot 4 \\
2 \cdot 5 \\
2 \cdot 8\end{array}$ & $\begin{array}{l}\text { SBP decreased } \\
\text { significantly; FG } \\
\text { increased } \\
\text { significantly }\end{array}$ & 4 \\
\hline Ganjali et al., 2016(93) & Iran & 45 & 58 & $\begin{array}{l}37.6 \\
(\mathrm{SD} 6.9)\end{array}$ & $25-58$ & $\begin{array}{l}\text { Quasi- } \\
\text { experimental }\end{array}$ & $\begin{array}{l}\text { HDL } \\
\text { TAG } \\
\text { FG }\end{array}$ & $\begin{array}{l}\text { Obese: } 37.2 \mathrm{mg} / \mathrm{dl} \\
\text { Normal: } 39.1 \mathrm{mg} / \mathrm{dl} \\
\text { Obese: } 263 \mathrm{mg} / \mathrm{dl} \\
\text { Normal: } 190 \mathrm{mg} / \mathrm{dl} \\
\text { Obese: } 98.86 \mathrm{mg} / \mathrm{dl} \\
\text { Normal: } 94.6 \mathrm{mg} / \mathrm{dl}\end{array}$ & $\begin{array}{c}8 \cdot 8 \\
8.7 \\
193 \cdot 6 \\
131 \cdot 2 \\
18 \cdot 68 \\
11.17\end{array}$ & $\begin{array}{l}\text { Obese: } 41 \mathrm{mg} / \mathrm{dl} \\
\text { Normal: } 40.1 \mathrm{mg} / \mathrm{dl} \\
\text { Obese: } 255 \mathrm{mg} / \mathrm{dl} \\
\text { Normal: } 163 \mathrm{mg} / \mathrm{dl} \\
\text { Obese: } 93.68 \mathrm{mg} / \mathrm{dl} \\
\text { Normal: } 85.69 \mathrm{mg} / \mathrm{dl}\end{array}$ & $\begin{array}{c}6.1 \\
7.9 \\
177 \cdot 4 \\
97 \\
11.66 \\
7.32\end{array}$ & $\begin{array}{l}\text { FG significantly } \\
\text { decreased in the } \\
\text { normal weight group; } \\
\text { HDL significantly } \\
\text { increased in the } \\
\text { obese group }\end{array}$ & 4 \\
\hline Sezen et al., 2016 ${ }^{(94)}$ & Turkey & 70 & 100 & $\begin{array}{l}37 \\
(\mathrm{SD} 7)\end{array}$ & & Prospective & SBP & $120 \cdot 2 \mathrm{mmHg}$ & $11 \cdot 6$ & $121.2 \mathrm{mmHg}$ & $10 \cdot 2$ & $\begin{array}{l}\text { No significant changes } \\
\text { in SBP }\end{array}$ & 4 \\
\hline Kiyani et al., $2017^{(95)}$ & Pakistan & 80 & 62.5 & $20 \cdot 5$ & $18-24$ & $\begin{array}{l}\text { Prospective } \\
\quad \text { observational }^{*}\end{array}$ & $\begin{array}{l}\text { HDL } \\
\text { TAG } \\
\text { FG }\end{array}$ & $\begin{array}{l}1.2 \mathrm{mmol} / \mathrm{l} \\
1.4 \mathrm{mmol} / \mathrm{l} \\
72.6 \mathrm{mg} / \mathrm{dl}\end{array}$ & $\begin{array}{r}0.3 \\
0.5 \\
12 \cdot 5\end{array}$ & $\begin{array}{l}1.1 \mathrm{mmol} / \mathrm{l} \\
1.2 \mathrm{mmol} / \mathrm{l} \\
57.9 \mathrm{mg} / \mathrm{dl}\end{array}$ & $\begin{array}{r}0.3 \\
0.5 \\
10 \cdot 7\end{array}$ & $\begin{array}{l}\text { Significant decline in } \\
\text { FG and TAG; } \\
\text { Significant reduction } \\
\text { in HDL }\end{array}$ & 4 \\
\hline $\begin{array}{l}\text { AbdulKareem et al., } \\
2017^{(96)}\end{array}$ & Iraq & 12 & 25 & $\begin{array}{c}37.5 \\
(\mathrm{SD} 10.8)\end{array}$ & $24-57$ & Case-control & $\begin{array}{l}\text { HDL } \\
\text { TAG } \\
\text { FG }\end{array}$ & $\begin{array}{l}49.58 \mathrm{mg} / \mathrm{dl} \\
92 \mathrm{mg} / \mathrm{dl} \\
86.25 \mathrm{mg} / \mathrm{dl}\end{array}$ & $\begin{array}{r}2.96 \\
10.83 \\
4.06\end{array}$ & $\begin{array}{l}53.25 \mathrm{mg} / \mathrm{dl} \\
94.83 \mathrm{mg} / \mathrm{dl} \\
63.17 \mathrm{mg} / \mathrm{dl}\end{array}$ & $\begin{array}{l}2.496 \\
9.67 \\
2 \cdot 51\end{array}$ & $\begin{array}{l}\text { Significant decrease in } \\
\text { FG and significant } \\
\text { increase in HDL and } \\
\text { TAG (healthy } \\
\text { subjects) }\end{array}$ & 4 \\
\hline $\begin{array}{l}\text { Alsubheen et al., } \\
2017^{(97)}\end{array}$ & Canada & 9 & 100 & $\begin{array}{c}32 \cdot 2 \\
(\mathrm{SD} 7 \cdot 8)\end{array}$ & & $\begin{array}{l}\text { Prospective } \\
\text { observational }^{\star}\end{array}$ & SBP & $120 \mathrm{mmHg}$ & 11 & $109 \mathrm{mmHg}$ & 12 & $\begin{array}{l}\text { Significant decrease in } \\
\text { SBP }\end{array}$ & 4 \\
\hline Bakki et al., 2017(98) & Nigeria & 75 & $62 \cdot 6$ & $\begin{array}{l}25 \\
(\mathrm{SD} 2)\end{array}$ & $18-30$ & Cross-sectional & $\begin{array}{l}\text { HDL } \\
\text { TAG } \\
\text { FG }\end{array}$ & $\begin{array}{l}1.4 \mathrm{mmol} / \mathrm{l} \\
1.2 \mathrm{mmol} / \mathrm{l} \\
4.0 \mathrm{mmol} / \mathrm{l}\end{array}$ & $\begin{array}{l}0.3 \\
0.4 \\
0.5\end{array}$ & $\begin{array}{l}1.3 \mathrm{mmol} / / \\
1.2 \mathrm{mmol} / \mathrm{l} \\
4.7 \mathrm{mmol} / /\end{array}$ & $\begin{array}{l}0.2 \\
0.3 \\
0.9\end{array}$ & $\begin{array}{l}\text { No significant changes } \\
\text { in TAG Significant } \\
\text { increase in HDL } \\
\text { Slight significant } \\
\text { increase in FG }\end{array}$ & 4 \\
\hline Khan et al., 2017(99) & Pakistan & 35 & 51 & $\begin{array}{c}21.66 \\
(\mathrm{SD} 0.7)\end{array}$ & $21-23$ & $\begin{array}{l}\text { Prospective } \\
\quad \text { observational }^{\star}\end{array}$ & $\begin{array}{l}\text { WC } \\
\text { HDL } \\
\text { TAG } \\
\text { FG } \\
\text { SBP }\end{array}$ & $\begin{array}{l}79.9 \mathrm{~cm} \\
55.88 \mathrm{mg} / \mathrm{dl} \\
87.76 \mathrm{mg} / \mathrm{dl} \\
88.79 \mathrm{mg} / \mathrm{dl} \\
113.08 \mathrm{mmHg}\end{array}$ & $\begin{array}{c}10 \cdot 18 \\
13 \cdot 73 \\
37 \cdot 87 \\
9 \cdot 1 \\
10 \cdot 52\end{array}$ & $\begin{array}{l}79.74 \mathrm{~cm} \\
49.82 \mathrm{mg} / \mathrm{dl} \\
79.82 \mathrm{mg} / \mathrm{dl} \\
87.2 \mathrm{mg} / \mathrm{dl} \\
113.56 \mathrm{mmHg}\end{array}$ & $\begin{array}{c}10.33 \\
10.09 \\
34.54 \\
6.35 \\
9.5\end{array}$ & $\begin{array}{l}\text { Mean HDL decreased } \\
\text { significantly }\end{array}$ & 4 \\
\hline $\begin{array}{l}\text { Malekmakan et al., } \\
2017^{(100)}\end{array}$ & Iran & 93 & $52 \cdot 7$ & $\begin{array}{c}37 \cdot 2 \\
(\mathrm{SD} 7.9)\end{array}$ & $25-57$ & $\begin{array}{l}\text { Semi- } \\
\text { experimental } \\
\text { study }\end{array}$ & $\begin{array}{l}\text { WC } \\
\text { SBP }\end{array}$ & $\begin{array}{l}89.1 \mathrm{~cm} \\
101.7 \mathrm{mmHg}\end{array}$ & $\begin{array}{l}11 \cdot 1 \\
12 \cdot 9\end{array}$ & $\begin{array}{l}87.5 \mathrm{~cm} \\
99.4 \mathrm{mmHg}\end{array}$ & $\begin{array}{l}11 \cdot 1 \\
12 \cdot 7\end{array}$ & $\begin{array}{l}\text { WC and SBP } \\
\text { significantly } \\
\text { decreased }\end{array}$ & 4 \\
\hline
\end{tabular}


Table 1. Continued

\begin{tabular}{|c|c|c|c|c|c|c|c|c|c|c|c|c|c|}
\hline \multirow{2}{*}{$\begin{array}{l}\text { Study, publication } \\
\text { year }\end{array}$} & \multirow[b]{2}{*}{ Country } & \multicolumn{2}{|c|}{ Sample size } & \multicolumn{2}{|c|}{ Age (years) } & \multirow[b]{2}{*}{ Study design } & \multirow{2}{*}{$\begin{array}{c}\text { Examined } \\
\text { markers }\end{array}$} & \multicolumn{2}{|c|}{ Before Ramadan $\dagger$} & \multicolumn{2}{|c|}{ End of Ramadan $†$} & \multirow{2}{*}{$\begin{array}{l}\text { Results (after } \\
\text { Ramadan month } \\
\text { compared with before) }\end{array}$} & \multirow{2}{*}{$\begin{array}{l}\text { Total } \\
\text { quality } \\
\text { score }\end{array}$} \\
\hline & & $n$ & $\%$ Male & Mean & Range & & & Mean & SD & Mean & SD & & \\
\hline $\begin{array}{l}\text { Norouzy et al., } \\
2017^{(101)}\end{array}$ & Iran & 12 & 50 & $\begin{array}{l}54.6 \\
\text { (sD 4) }\end{array}$ & & $\begin{array}{l}\text { Prospective } \\
\text { observational }\end{array}$ & SBP & $119.5 \mathrm{mmHg}$ & 6 & $117.6 \mathrm{mmHg}$ & 9 & $\begin{array}{l}\text { No significant } \\
\text { difference in SBP }\end{array}$ & 4 \\
\hline $\begin{array}{l}\text { Ongsara et al., } \\
2017^{(102)}\end{array}$ & Thailand & 65 & 32 & $\begin{array}{r}20 \cdot 82 \\
(\mathrm{SD} 1 \cdot 1)\end{array}$ & $19-24$ & $\begin{array}{l}\text { Prospective } \\
\text { observational }\end{array}$ & $\begin{array}{l}\text { WC } \\
\text { HDL } \\
\text { TAG } \\
\text { FG } \\
\text { SBP }\end{array}$ & $\begin{array}{l}\text { Male: } 79.83 \mathrm{~cm} \\
\text { Female: } 63.45 \mathrm{~cm} \\
\text { Male: } 1.26 \mathrm{mmol} / / \\
\text { Female: } 1.47 \mathrm{mmol} / \mathrm{l} \\
\text { Male: } 0.92 \mathrm{mmol} / \mathrm{l} \\
\text { Female: } 0.83 \mathrm{mmol} / / \\
\text { Male: } 5.34 \mathrm{mmol} / / \\
\text { Female: } 4.83 \mathrm{mmol} / / \\
\text { Male: } 126.76 \mathrm{mmHg} \\
\text { Female: } \\
\quad 107.14 \mathrm{mmHg}\end{array}$ & $\begin{array}{r}12 \cdot 4 \\
6 \cdot 66 \\
0.24 \\
0.33 \\
0.36 \\
0.28 \\
0.51 \\
0.38 \\
15.38 \\
7.05\end{array}$ & $\begin{array}{l}\text { Male: } 76.5 \mathrm{~cm} \\
\text { Female: } 65.44 \mathrm{~cm} \\
\text { Male: } 1.42 \mathrm{mmol} / / \\
\text { Female: } 1.51 \mathrm{mmol} / \mathrm{l} \\
\text { Male: } 1.02 \mathrm{mmol} / / \\
\text { Female: } 0.79 \mathrm{mmol} / / \\
\text { Male: } 5.27 \mathrm{mmol} / / \\
\text { Female: } 4.9 \mathrm{mmol} / / \\
\text { Male: } 126.95 \mathrm{mmHg} \\
\text { Female: } \\
\quad 107.36 \mathrm{mmHg}\end{array}$ & $\begin{array}{l}10 \cdot 84 \\
7 \\
0.3 \\
0.35 \\
0.3 \\
0.28 \\
0.41 \\
0.41 \\
14.54 \\
9.86\end{array}$ & $\begin{array}{l}\text { No significant changes } \\
\text { in WC, BP, TAG or } \\
\text { HDL for either sex }\end{array}$ & 4 \\
\hline $\begin{array}{l}\text { Mohammadzade } \\
\text { et al., 2017 (103) }\end{array}$ & Iran & 30 & 100 & $\begin{array}{r}29.44 \\
(\mathrm{SD} 7.4)\end{array}$ & $20-35$ & $\begin{array}{l}\text { Prospective } \\
\text { observational }\end{array}$ & $\begin{array}{l}\text { WC } \\
\text { HDL } \\
\text { TAG } \\
\text { FG } \\
\text { SBP }\end{array}$ & $\begin{array}{l}96.48 \mathrm{~cm} \\
33.83 \mathrm{mg} / \mathrm{dl} \\
152.55 \mathrm{mg} / \mathrm{dl} \\
98.58 \mathrm{mg} / \mathrm{dl} \\
124.7 \mathrm{mmHg}\end{array}$ & $\begin{array}{r}11.38 \\
8.53 \\
64.35 \\
7.04 \\
4\end{array}$ & $\begin{array}{l}95.31 \mathrm{~cm} \\
47.59 \mathrm{mg} / \mathrm{dl} \\
123.83 \mathrm{mg} / \mathrm{dl} \\
81 \mathrm{mg} / \mathrm{dl} \\
121.6 \mathrm{mmHg}\end{array}$ & $\begin{array}{c}10 \cdot 62 \\
6 \cdot 7 \\
53 \cdot 44 \\
4.97 \\
6\end{array}$ & $\begin{array}{l}\text { No significant change } \\
\text { in SBP; significantly } \\
\text { decreased TAG and } \\
\text { FG;-significantly } \\
\text { increased HDL }\end{array}$ & 4 \\
\hline $\begin{array}{l}\text { Abubakar et al., } \\
2018^{(104)}\end{array}$ & Pakistan & 60 & NR & $\begin{array}{c}34 \cdot 3 \\
(\mathrm{SD} 8 \cdot 6)\end{array}$ & & $\begin{array}{l}\text { Prospective } \\
\quad \text { observational }^{*}\end{array}$ & $\begin{array}{l}\text { HDL } \\
\text { TAG } \\
\text { FG }\end{array}$ & $\begin{array}{l}1.08 \mathrm{mmol} / / \\
1.33 \mathrm{mmol} / / \\
5.58 \mathrm{mmol} / /\end{array}$ & $\begin{array}{l}0.35 \\
0.75 \\
1.17\end{array}$ & $\begin{array}{l}1.11 \mathrm{mmol} / / \\
1.45 \mathrm{mmol} / / \\
5.61 \mathrm{mmol} / /\end{array}$ & $\begin{array}{l}0.39 \\
0.99 \\
0.97\end{array}$ & $\begin{array}{l}\text { No significant change } \\
\text { in TAG, HDL or FG }\end{array}$ & 2 \\
\hline $\begin{array}{l}\text { Al-Barha \& Aljaloud, } \\
\qquad 2018^{(105)}\end{array}$ & $\begin{array}{l}\text { Saudi } \\
\text { Arabia }\end{array}$ & 44 & 100 & $\begin{array}{l}27 \cdot 7 \\
(\text { SD } 5 \cdot 8)\end{array}$ & 18-39 & $\begin{array}{l}\text { Quasi- } \\
\text { experimental } \\
\text { before/after } \\
\text { study }\end{array}$ & $\begin{array}{l}\text { WC } \\
\text { FG } \\
\text { SBP }\end{array}$ & $\begin{array}{l}82.9 \mathrm{~cm} \\
74.60 \mathrm{mg} / \mathrm{dl} \\
109.6 \mathrm{mmHg}\end{array}$ & $\begin{array}{c}10 \cdot 9 \\
9\end{array}$ & $\begin{array}{l}81.8 \mathrm{~cm} \\
81.52 \mathrm{mg} / \mathrm{dl} \\
111.8 \mathrm{mmHg}\end{array}$ & $\begin{array}{l}10 \cdot 5 \\
10 \cdot 8\end{array}$ & $\begin{array}{l}\text { FG and SBP were } \\
\text { slightly but } \\
\text { significantly elevated }\end{array}$ & 4 \\
\hline $\begin{array}{l}\text { Nachvak et al., } \\
2018^{(19)}\end{array}$ & Iran & 152 & 100 & $\begin{array}{r}39.35 \\
(\mathrm{SD} 11)\end{array}$ & $21-63$ & Observational & $\begin{array}{l}\text { HDL } \\
\text { TAG } \\
\text { FG }\end{array}$ & $\begin{array}{l}44.70 \mathrm{mg} / \mathrm{dl} \\
151.44 \mathrm{mg} / \mathrm{dl} \\
80.17 \mathrm{mg} / \mathrm{dl}\end{array}$ & $\begin{array}{r}7 \cdot 9 \\
85 \cdot 2 \\
19 \cdot 3\end{array}$ & $\begin{array}{l}45.59 \mathrm{mg} / \mathrm{dl} \\
140.44 \mathrm{mg} / \mathrm{dl} \\
72.06 \mathrm{mg} / \mathrm{dl}\end{array}$ & $\begin{array}{c}9 \\
75 \cdot 2 \\
8 \cdot 4\end{array}$ & $\begin{array}{l}\text { HDL levels increased } \\
\text { significantly; } \\
\text { significant decrease } \\
\text { in TAG and FG }\end{array}$ & 4 \\
\hline $\begin{array}{l}\text { Prasetya \& } \\
\text { Sepwarobol, } \\
2018^{(106)}\end{array}$ & Thailand & 27 & 100 & $\begin{array}{l}24 \cdot 3 \\
(\mathrm{sD} 3 \cdot 7)\end{array}$ & $19-40$ & $\begin{array}{l}\text { Prospective } \\
\text { observational }^{*}\end{array}$ & $\begin{array}{l}\text { WC } \\
\text { HDL } \\
\text { TAG } \\
\text { FG }\end{array}$ & $\begin{array}{l}81.82 \mathrm{~cm} \\
52.84 \mathrm{mg} / \mathrm{dl} \\
90.59 \mathrm{mg} / \mathrm{dl} \\
4.83 \mathrm{mmol} / \mathrm{l}\end{array}$ & $\begin{array}{c}7 \cdot 73 \\
13 \cdot 2 \\
62 \cdot 43 \\
0 \cdot 36\end{array}$ & $\begin{array}{l}78.82 \mathrm{~cm} \\
48.89 \mathrm{mg} / \mathrm{dl} \\
77.37 \mathrm{mg} / \mathrm{dl} \\
4.87 \mathrm{mmol} / \mathrm{l}\end{array}$ & $\begin{array}{r}7.96 \\
11.91 \\
50 \cdot 14 \\
0.35\end{array}$ & $\begin{array}{l}\text { Reductions in WC and } \\
\mathrm{HDL} \text {; no significant } \\
\text { change in FG }\end{array}$ & 4 \\
\hline $\begin{array}{l}\text { Rahbar et al., } \\
2019^{(107)}\end{array}$ & Iran & 34 & 100 & $\begin{array}{l}35 \\
\text { (sD 11) }\end{array}$ & $16-64$ & $\begin{array}{l}\text { Prospective } \\
\text { observational }^{*}\end{array}$ & $\begin{array}{l}\text { WC } \\
\text { HDL } \\
\text { TAG }\end{array}$ & $\begin{array}{l}91.97 \mathrm{~cm} \\
43.79 \mathrm{mg} / \mathrm{dl} \\
143.56 \mathrm{mg} / \mathrm{dl}\end{array}$ & $\begin{array}{r}11.71 \\
5.49 \\
47.64\end{array}$ & $\begin{array}{l}91.45 \mathrm{~cm} \\
48.38 \mathrm{mg} / \mathrm{dl} \\
120.33 \mathrm{mg} / \mathrm{dl}\end{array}$ & $\begin{array}{r}11.59 \\
9.39 \\
42.67\end{array}$ & $\begin{array}{l}\text { WC and TAG } \\
\text { significantly } \\
\text { decreased; HDL } \\
\text { levels significantly } \\
\text { increased }\end{array}$ & 4 \\
\hline
\end{tabular}


Table 2. Characteristics and pooled analyses of included studies for each metabolic syndrome component

\begin{tabular}{lcccccccc}
\hline Component & $K^{*}$ & $N \dagger$ & $\begin{array}{c}\text { Number of } \\
\text { countries }\end{array}$ & $\begin{array}{c}\text { Mean age } \\
\text { (years) }\end{array}$ & $\begin{array}{c}\text { Overall \% } \\
\text { male }\end{array}$ & $\begin{array}{c}\text { Fasting time } \\
(\mathrm{min} / \mathrm{d})\end{array}$ & Hedges' $g$ & \multicolumn{1}{c}{$95 \% \mathrm{Cl}$} \\
\hline WC & 24 & 1557 & 14 & 31.5 & 58.7 & 841 & -0.312 & $-0.387,-0.236$ \\
FG & 51 & 2318 & 19 & 30.3 & 66 & 817 & -0.101 & $-0.260,0.004$ \\
TAG & 63 & 2862 & 21 & 31 & 67 & 820 & -0.088 & $-0.171,-0.004$ \\
HDL & 57 & 2771 & 19 & 31.5 & 66 & 830 & 0.150 & $0.064,0.236$ \\
SBP & 22 & 1172 & 13 & 33 & 63 & 876 & -0.239 & $-0.372,-0.106$ \\
\hline
\end{tabular}

FG, fasting plasma glucose; SBP, systolic blood pressure; WC, waist circumference.

${ }^{*} K$ : denotes number of studies.

$\dagger N$ : denotes number of participants.

\begin{tabular}{|c|c|c|c|c|c|c|c|}
\hline \multirow[t]{2}{*}{ Study name } & \multicolumn{7}{|c|}{ Statistics for each study } \\
\hline & $\begin{array}{l}\text { Hedges' } \\
g\end{array}$ & $\begin{array}{l}\text { Standard } \\
\text { error }\end{array}$ & Variance & $\begin{array}{l}\text { Lower } \\
\text { limit }\end{array}$ & $\begin{array}{l}\text { Upper } \\
\text { limit }\end{array}$ & $Z$-Value & $P$-Value \\
\hline Norouzy et al. 2010 & -0.214 & 0.065 & 0.004 & -0.342 & -0.087 & -3.294 & 0.001 \\
\hline Yucel et al. 2004 & 0.036 & 0.159 & 0.025 & -0.276 & 0.348 & 0.227 & 0.821 \\
\hline Malekmakan et al 2017 & -0.350 & 0.106 & 0.011 & -0.557 & -0.142 & -3.299 & 0.001 \\
\hline Mohammadzade et al. 2011 & -0.348 & 0.183 & 0.034 & -0.707 & 0.011 & -1.898 & 0.058 \\
\hline Shariatpanahi et al. 2007 & -0.463 & 0.140 & 0.020 & -0.737 & -0.188 & -3.303 & 0.001 \\
\hline Sayedda et al. 2012 & -0.834 & 0.252 & 0.063 & $-1 \cdot 327$ & -0.340 & -3.309 & 0.001 \\
\hline Al-Barha \& Aljaloud 2018 & -0.286 & 0.151 & 0.023 & -0.582 & 0.010 & -1.892 & 0.058 \\
\hline Prasetya \& Sapwarobol 2018 & -0.693 & 0.209 & 0.044 & $-1 \cdot 103$ & -0.282 & -3.309 & 0.001 \\
\hline Shehab et al 2012 & -0.268 & 0.130 & 0.017 & -0.522 & -0.013 & -2.063 & 0.039 \\
\hline Celik et al. 2014 & -0.537 & 0.162 & 0.026 & -0.855 & -0.219 & -3.306 & 0.001 \\
\hline Ongsara et al 2017 & 0.235 & 0.124 & 0.015 & -0.009 & 0.478 & 1.888 & 0.059 \\
\hline Kassab et al 2004 & -0.280 & 0.148 & 0.022 & -0.570 & 0.010 & -1.892 & 0.059 \\
\hline Kassab et al 2003 & -0.286 & 0.151 & 0.023 & -0.582 & 0.010 & -1.892 & 0.058 \\
\hline Saleh et al. 2005 & -0.441 & 0.134 & 0.018 & -0.703 & -0.179 & $-3 \cdot 302$ & 0.001 \\
\hline Fakhrzadeh et al. 2003 & -0.354 & 0.107 & 0.011 & -0.564 & -0.144 & -3.299 & 0.001 \\
\hline Faris et al. 2012 & -0.268 & 0.142 & 0.020 & -0.546 & 0.010 & -1.891 & 0.059 \\
\hline Khan et al.2017 & -0.322 & 0.170 & 0.029 & -0.654 & 0.011 & -1.895 & 0.058 \\
\hline Narouzy et al. 2013 & -0.214 & 0.065 & 0.004 & -0.342 & -0.087 & -3.294 & 0.001 \\
\hline Rahbar et al:2019 & -0.383 & 0.174 & 0.030 & -0.723 & -0.042 & -2.200 & 0.028 \\
\hline López-Bueno et al. 2015 & -0.206 & 0.127 & 0.016 & -0.454 & 0.043 & -1.623 & 0.105 \\
\hline Rohin et al.2013 & -0.510 & 0.154 & 0.024 & -0.813 & -0.208 & $-3 \cdot 305$ & 0.001 \\
\hline Agoumi et al. 2013 & -0.463 & 0.140 & 0.020 & -0.737 & -0.188 & -3.303 & 0.001 \\
\hline McNeil et al 2014 & -0.234 & 0.218 & 0.047 & -0.661 & 0.193 & -1.073 & 0.283 \\
\hline Hassan \& Isawumi 2014 & -0.441 & 0.134 & 0.018 & -0.703 & -0.179 & $-3 \cdot 302$ & 0.001 \\
\hline & -0.312 & 0.038 & 0.001 & -0.387 & -0.236 & -8.114 & 0.000 \\
\hline
\end{tabular}

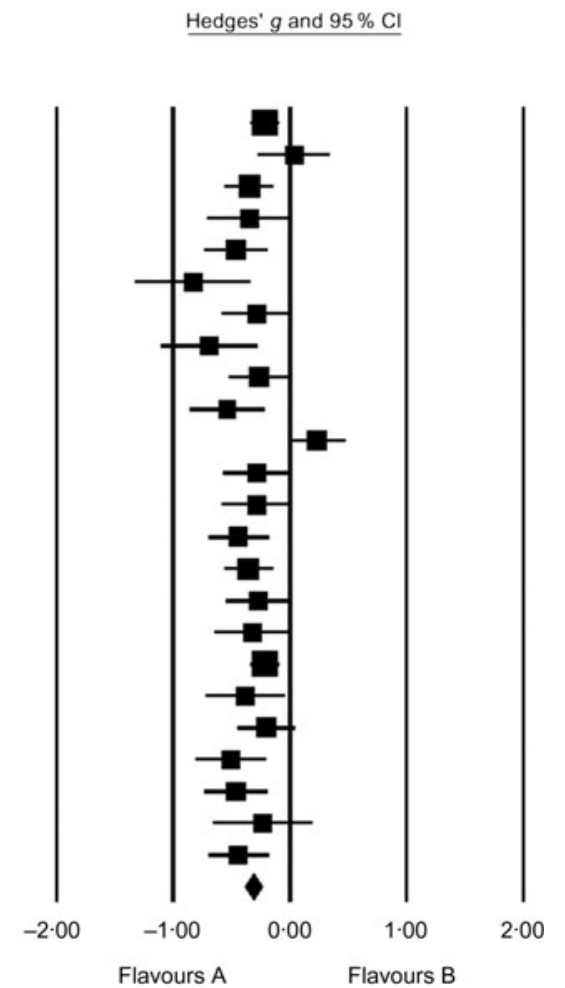

Fig. 2. According to Hedges' $g$ value with $95 \% \mathrm{Cl}$, small $(-0.312)$ significant reduction in waist circumference was induced by Ramadan fasting. Heterogeneity statistics: $95 \% \mathrm{Cl}-0.387,-0.236, I^{2}=49 \%$. Hedges' $g$ value is considered small when value $=0.2$, medium $=0.5$, large $=0.8$.

more studies were available to explore differences in findings among countries (Table 4).

\section{Waist circumference}

Age (online Supplementary Fig. S6) and fasting time/d (online Supplementary Fig. S7) were NS moderators for changes in WC. However, sex was significant in explaining variation in WC $(\beta=-0.20, P=0.03)$ (online Supplementary Fig. S8), suggesting that women experienced a larger change in WC than men during RDIF. Only Iran contributed three or more studies that measured WC change during RDIF $(K=7, N$ 783): Hedges' $g=-0 \cdot 275,95 \% \mathrm{CI}-0 \cdot 346,-0 \cdot 204, I^{2}=0 \cdot 0 \%$; online Supplementary Fig. S9).

\section{Fasting glucose}

Age $(\beta=0.005, P=0.05$; online Supplementary Fig. S10) and fasting time $/ \mathrm{d}(\beta=-0.001, P=0.001$; online Supplementary Fig. S11) had significant impacts as moderators for changes in FG, whereas sex had no significant impact on FG changes during Ramadan fasting (online Supplementary Fig. S12). Six countries contributed three or more studies that measured FG changes during Ramadan month (online Supplementary Fig. S13): Iran $(K=15, N$ 828, Hedges' $g=-0.173,95 \%$ CI -0.348 , $\left.0.002, I^{2}=81.93 \%\right)$, Saudi Arabia $(K=5, N 218$, Hedges' $g=0 \cdot 075,95 \%$ CI $\left.-0 \cdot 240,0 \cdot 390, I^{2}=80 \cdot 41 \%\right)$, Turkey $(K=4$, $N$ 146, Hedges' $g=-0.069, \quad 95 \% \quad$ CI $-0.735, \quad 0.596$, $\left.I^{2}=93.05 \%\right)$, Tunisia $(K=4, N 109$, Hedges' $g=0 \cdot 180,95 \%$ CI $\left.-0.271,0 \cdot 630, I^{2}=81.6 \%\right)$, Jordan ( $K=3, N$ 186, Hedges' 


\begin{tabular}{|c|c|}
\hline \multicolumn{2}{|l|}{ Study name } \\
\hline & $\begin{array}{c}\text { Hedges' } \\
g\end{array}$ \\
\hline AbdulKareem et al. 2017 & $-0-591$ \\
\hline Abubakar et al 2018 & 0.046 \\
\hline Adlouni et al. 1997 & -0.627 \\
\hline AJ-Barha and Aljaloud 2018 & 0.299 \\
\hline AlNahari and Kouja 2014 & -0.392 \\
\hline Al-Numair 2006 & -0.295 \\
\hline Asgary et al. 2000 & -0.118 \\
\hline Azizi \& Rasouli 1987 & 1.518 \\
\hline Babaei et al. 2016 & 0.398 \\
\hline Bahammam et al. 2016 & 0.211 \\
\hline Bahiji et al. 2013 & 0.567 \\
\hline Bakki et al. 2017 & 0.392 \\
\hline Barkia et al 2011 & 0.382 \\
\hline Bilto 1998 & -0.220 \\
\hline Celik et al. 2014 & 0.537 \\
\hline El Ati et al 1995 & 0.483 \\
\hline Esmaeilzadeh \& Borne 2016 & 0.758 \\
\hline Fakhrzadeh et al. 2003 & -0.217 \\
\hline Farshidfar et al. 2006 & -0.808 \\
\hline Feizollahzadeh et al. 2014 & 0.406 \\
\hline Furuncuoglu et al 2007 & -0.560 \\
\hline Hosseini \& Hejazi 2015 & 0.249 \\
\hline Hosseini et al. 2013 & -0.253 \\
\hline Ibrahim et al 2008 & -0.543 \\
\hline Kassab et al. 2003 & -0.286 \\
\hline Kassab et al 2004 & -0.280 \\
\hline Khan et al. 2017 & -0.322 \\
\hline Kiyani et al. 2015 & -0.379 \\
\hline Lamine et al.2006 & 0.348 \\
\hline Larijani et al 2003 & -0.313 \\
\hline Maislos et al. 1998 & 0.409 \\
\hline Mansi 2007 & -0.226 \\
\hline Mansi \& Amneh 2007 & -0.293 \\
\hline McNeil et al 2014 & -0.834 \\
\hline Mohajeri et al 2013 & -0.356 \\
\hline Mohammadzade et al. 2011 & -0.651 \\
\hline Nachvak et al. 2018 & -0.271 \\
\hline Ongsara et al 2017 & 0.423 \\
\hline Oukerro et al.2013 & -0.432 \\
\hline Prasetya \& Sapwarobol 2018 & 0.076 \\
\hline Rahman et al. 2004 & -0.681 \\
\hline Ramadan 2002 & 0.483 \\
\hline Saleh et al. 2005 & -0.244 \\
\hline Salehi \& Neghab 2007 & -0.509 \\
\hline Shariatpanahi et al. 2007 & -0.389 \\
\hline Sülü et al. 2008 & 0.517 \\
\hline Suriani et al. 2015 & -0.252 \\
\hline Unalacak et al 2011 & -0.834 \\
\hline Zahid J. Mohammed 2011 & -0.253 \\
\hline Ziaee et al. 2006 & -0.376 \\
\hline & $-0-101$ \\
\hline
\end{tabular}

Statistics for each study

\begin{tabular}{|c|c|c|c|c|c|c|}
\hline$\underset{g}{\text { dges' }}$ & $\begin{array}{c}\text { Standard } \\
\text { error }\end{array}$ & Variance & $\begin{array}{l}\text { Lower } \\
\text { limit }\end{array}$ & $\begin{array}{c}\text { Upper } \\
\text { limit }\end{array}$ & Z-Value & $P$-Valu \\
\hline$-0-591$ & 0.294 & 0.087 & $-1 \cdot 168$ & -0.014 & -2.008 & 0.045 \\
\hline 0.046 & 0.128 & 0.016 & -0.204 & 0.296 & 0.360 & 0.719 \\
\hline-0.627 & 0.189 & 0.036 & -0.998 & -0.255 & $-3 \cdot 308$ & 0.001 \\
\hline 0.299 & 0.151 & 0.023 & 0.002 & 0.596 & 1.972 & 0.049 \\
\hline-0.392 & 0.198 & 0.039 & -0.779 & -0.004 & $-1 \cdot 980$ & 0.048 \\
\hline 0.295 & 0.150 & 0.022 & -0.589 & -0.002 & $-1 \cdot 971$ & 0.049 \\
\hline-118 & 0.145 & 0.021 & -0.403 & 0.167 & -0.811 & 0.417 \\
\hline 1.518 & 0.468 & 0.219 & 0.601 & 2.434 & 3.246 & 0.001 \\
\hline 0.398 & 0.170 & 0.029 & 0.065 & 0.730 & $2 \cdot 343$ & 0.019 \\
\hline 0.211 & 0.112 & 0.013 & -0.008 & 0.431 & $1-887$ & 0.059 \\
\hline 0.567 & 0.218 & 0.048 & 0.140 & 0.995 & 2.603 & 0.009 \\
\hline 0.392 & 0.119 & 0.014 & $0-159$ & 0.624 & 3.300 & 0.001 \\
\hline 0.382 & 0.201 & 0.040 & -0.012 & 0.776 & 1.901 & 0.057 \\
\hline-0.220 & $0-116$ & & -0.448 & & & 059 \\
\hline 0.537 & 0.162 & 0.026 & 0.219 & 0.855 & 3.306 & \\
\hline 0.483 & 0.252 & 0.064 & -0.012 & 0.977 & 1.914 & .056 \\
\hline 0.758 & 0.289 & 0.084 & 0.190 & $1 \cdot 325$ & $2 \cdot 618$ & 0.009 \\
\hline-0.217 & 0.105 & 0.011 & -0.423 & -0.010 & $-2 \cdot 060$ & 0.039 \\
\hline-0.808 & 0.244 & 0.060 & -1.287 & -0.330 & $-3 \cdot 310$ & 0.001 \\
\hline 0.406 & 0.123 & 15 & 0.165 & 0.648 & 3-301 & 0.001 \\
\hline-0.560 & 0.169 & 0.029 & -0.891 & -0.228 & $-3 \cdot 306$ & 0.001 \\
\hline 0.249 & 0.197 & 0.039 & -0.137 & 0.635 & 1.264 & 0.206 \\
\hline-0.253 & 0.284 & 80 & -0.809 & 302 & -0.894 & 0.371 \\
\hline-0.543 & 0.272 & 74 & -1.076 & $-0-011$ & $-2 \cdot 000$ & 0.045 \\
\hline-0.286 & 0.151 & 3 & -0.582 & 0.010 & -1.892 & 058 \\
\hline-0.280 & 0.148 & & -0.570 & 10 & -1.892 & 059 \\
\hline-0.322 & 0.170 & 0.029 & -0.654 & 0.011 & -1.895 & 58 \\
\hline-0.379 & 0.115 & 0.013 & -0.603 & -0.154 & $-3 \cdot 300$ & 001 \\
\hline 0.348 & 0.183 & 0.034 & -0.011 & 0.707 & 1.898 & 0.058 \\
\hline-0.313 & 0.095 & 0.009 & -0.499 & -0.127 & $-3-297$ & 0.001 \\
\hline 0.409 & 0.215 & 0.046 & -0.012 & 0.829 & 1.904 & 0.057 \\
\hline-0.226 & 0.120 & 14 & -0.461 & 0.009 & -1.888 & 0.059 \\
\hline-0.293 & 0.155 & 0.024 & -0.596 & 0.010 & -1.893 & 0.058 \\
\hline-0.834 & 0.252 & 0.063 & $-1 \cdot 327$ & -0.340 & $-3 \cdot 309$ & 0.001 \\
\hline-0.356 & 0.134 & 0.018 & -0.618 & -0.094 & -2.663 & 0.008 \\
\hline-0.651 & 0.197 & & -1.036 & -0.265 & $-3 \cdot 309$ & 0.001 \\
\hline-0.271 & 0.08 & 7 & -0.432 & -0.110 & -3.296 & 0.001 \\
\hline 0.423 & 0.128 & 16 & $0-172$ & 4 & 3-301 & 001 \\
\hline-0.432 & 0.166 & 0.028 & -0.758 & -0.105 & -2.593 & 0.010 \\
\hline 0.076 & 0.187 & 0.035 & -0.291 & 0.443 & 0.407 & 0.684 \\
\hline$-0-681$ & 0.240 & 0.058 & $-1 \cdot 152$ & -0.211 & $-2-837$ & 0.005 \\
\hline 0.483 & 0.252 & 0.064 & -0.012 & 0.977 & 1.914 & 0.056 \\
\hline-0.244 & 0.129 & 0.017 & -0.498 & 0.009 & -1.889 & 0.059 \\
\hline-0.509 & 0.196 & 0.038 & -0.893 & -0.125 & -2.598 & 0.009 \\
\hline-0.389 & 0.138 & 0.019 & -0.660 & -0.119 & -2.819 & 0.005 \\
\hline $0-517$ & 0.156 & 0.024 & 0.210 & $0-823$ & 3.305 & 0.001 \\
\hline-0.252 & 0.11 & 0.012 & -0.468 & -0.037 & $-2 \cdot 297$ & 0.022 \\
\hline-0.834 & 0.2 & & $-1 \cdot 327$ & -0.340 & -3.309 & 0.001 \\
\hline 0.253 & 0.7 & 18 & -0.516 & 0.009 & $-1 \cdot 890$ & 0.059 \\
\hline 376 & 0.1 & 0.013 & -0.599 & -0.153 & $-3 \cdot 300$ & 0.001 \\
\hline 0-101 & 0.053 & 0.003 & -0.206 & 0.004 & -1.894 & 0.058 \\
\hline
\end{tabular}

Hedges' $g$ and $95 \% \mathrm{Cl}$

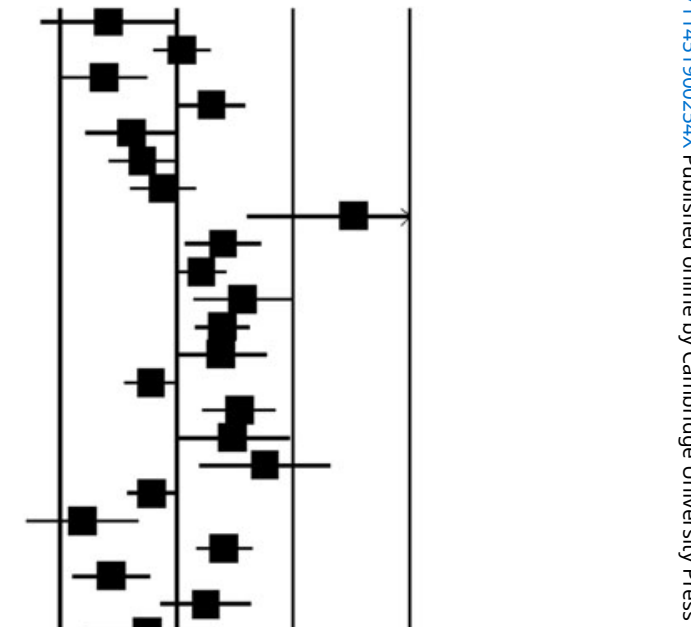

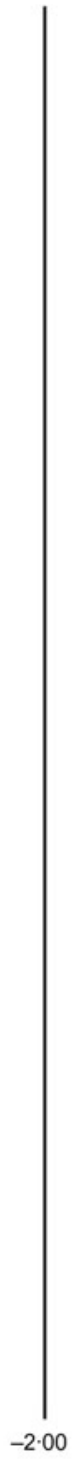

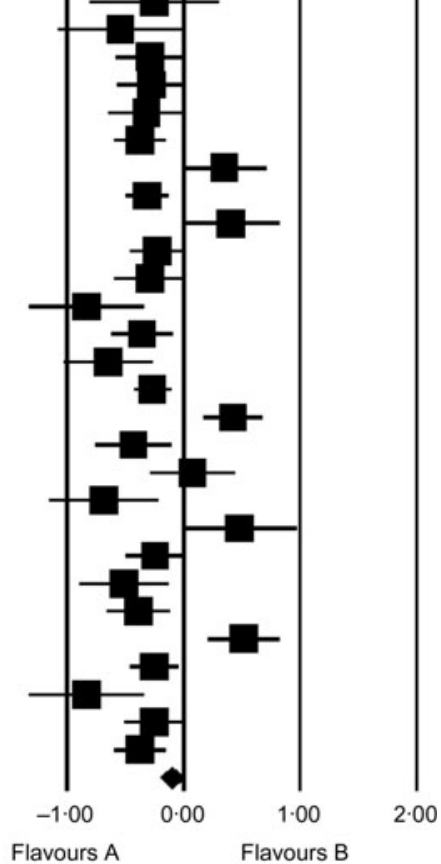

Fig. 3. According to Hedges' $g$ value with $95 \% \mathrm{Cl}$, small (-0.101) significant reduction in fasting glucose was induced by Ramadan fasting. Heterogeneity statistics: $95 \% \mathrm{Cl}-0.206,0.004, I^{2}=26.6 \%$. Hedges' $g$ value is considered small when value $=0.2$, medium $=0.5$, large $=0.8$.

$g=-0.239,95 \%$ CI $\left.-0.383,-0.095, I^{2}=0.0 \%\right)$ and Pakistan $(K=3, N$ 175, Hedges' $g=-0.215,95 \%$ CI $-0.494,0.064$, $\left.I^{2}=69 \cdot 8 \%\right)$.

\section{TAG}

Sex $(\beta=-0 \cdot 14, P=0 \cdot 01$; online Supplementary Fig. S14) and fasting time $/ \mathrm{d}(\beta=-0.005, \quad P=0.01 ;$ online Supplementary Fig. S15) had significant impacts as moderators for changes in TAG during RDIF, but age (online Supplementary Fig. S16) had no significant impact on TAG changes (Table 3). These findings suggested that women experienced a larger change in TAG than men during Ramadan month and that longer fasting time/d was associated with a greater reduction in TAG at the end of Ramadan. Six countries contributed three or more studies that measured TAG change during RDIF (online Supplementary Fig. S17): Iran $(K=20, N 1156$, Hedges' $g=-0.073,95 \%$ CI $\left.-0 \cdot 204,0 \cdot 058, I^{2}=78 \cdot 7 \%\right)$, Turkey $(K=6, N$ 210, Hedges' $g=-0.229,95 \%$ CI $\left.-0.458,0.001, I^{2}=63.4 \%\right)$, Jordan $(K=4$, $N 243$, Hedges' $g=-0 \cdot 244,95 \%$ CI $\left.-0 \cdot 370,-0 \cdot 117, I^{2}=0 \cdot 0 \%\right)$, Tunisia $(K=4, N 109$, Hedges' $g=-0 \cdot 116$, $95 \%$ CI $-0 \cdot 706$, $\left.0 \cdot 473, I^{2} 85 \cdot 6 \%\right)$, Pakistan $(K=3, N$ 175, Hedges' $g=-0 \cdot 212$, $95 \%$ CI $\left.-0.591,0 \cdot 168, I^{2}=83.5 \%\right)$ and Saudi Arabia $(K=3$, $N 94$, Hedges' $\left.g=0 \cdot 269,95 \% \mathrm{CI}-0 \cdot 366,0 \cdot 905, I^{2}=88 \cdot 5 \%\right)$. 


\begin{tabular}{|c|c|c|c|c|c|c|c|}
\hline \multirow[t]{2}{*}{ Study name } & \multicolumn{7}{|c|}{ Statistics for each study } \\
\hline & $\underset{g}{\text { Hedges' }}$ & $\begin{array}{l}\text { Standard } \\
\text { error }\end{array}$ & Variance & $\begin{array}{l}\text { Lower } \\
\text { limit }\end{array}$ & $\begin{array}{l}\text { Upper } \\
\text { limit }\end{array}$ & Z-Value & $P$-Valu \\
\hline Babaei et al 2016 & -0.369 & 0.169 & 0.028 & -0.700 & -0.038 & $-2 \cdot 186$ & 0.029 \\
\hline Akaberi et al 2014 & -0.043 & $0-150$ & 0.022 & -0.337 & 0.250 & -0.290 & $0-772$ \\
\hline Salehi \& Neghab 2007 & 0.361 & $0-190$ & 0.036 & -0.012 & 0.733 & 1.899 & 0.058 \\
\hline Sülũ et al 2008 & -0.400 & 0.152 & 0.023 & -0699 & -0.102 & -2.626 & 0.009 \\
\hline Sijavandi et al. 2015 & 0.140 & $0-106$ & 0.011 & -0.067 & 0.347 & 1.326 & $0-185$ \\
\hline Abubakar et al. 2018 & 0.136 & 0.128 & 0.016 & -0.115 & 0.387 & 1.063 & 0.288 \\
\hline Farshidfar et al 2006 & 0.165 & 0.211 & 0.045 & -0.249 & 0.580 & 0.782 & 0.434 \\
\hline Mohammadzade et al 2011 & -0.364 & $0-184$ & 0.034 & -0.724 & -0.003 & -1.977 & 0.048 \\
\hline Kiyani et al. 2015 & -0.454 & $0-116$ & 0.014 & -0.682 & -0.226 & -3.899 & 0.000 \\
\hline Asgary et al 2000 & -0.293 & $0-148$ & 0.022 & -0.584 & -0.003 & -1.980 & 0.048 \\
\hline Fedail et al. 1982 & -0.390 & 0.205 & 0.042 & -0.793 & 0.012 & -1.902 & 0.057 \\
\hline Suriani $e t$ al 2015 & -0.312 & $0-111$ & 0.012 & -0.529 & -0.095 & -2.815 & 0.005 \\
\hline Akhtaruzzaman et al 2014 & 0.098 & $0-184$ & 0.034 & -0.263 & 0.459 & 0.532 & 0.595 \\
\hline Ismail \& Haron 2014 & -0.475 & 0.185 & 0.034 & -0.837 & -0.112 & -2.563 & 0.010 \\
\hline Mohammed 2011 & -0.352 & 0.136 & 0.018 & -0618 & -0.085 & -2.587 & 0.010 \\
\hline Mansi 2007 & -0.226 & 0.120 & 0.014 & -0.461 & 0.009 & -1.888 & 0.059 \\
\hline Furuncuoglu et al 2007 & -0.426 & 0.164 & 0.027 & -0.747 & -0.104 & -2.592 & 0.010 \\
\hline Shariatpanahi et al 2007 & 0.166 & 0.134 & 0.018 & -0.096 & 0.429 & 1.243 & 0.214 \\
\hline AL-Numair 2006 & -0.295 & $0-150$ & 0.022 & -0.589 & -0.002 & -1.971 & 0.049 \\
\hline Aksungar et al 2007 & -0.300 & 0.159 & 0.025 & -0611 & 0.011 & -1.893 & 0.058 \\
\hline Mohajeri et al 2013 & -0.308 & 0.133 & 0.018 & -0.568 & -0.048 & $-2 \cdot 318$ & 0.020 \\
\hline Rahman et al 2004 & -0.429 & 0.225 & 0.051 & -0.871 & 0.012 & -1.907 & 0.057 \\
\hline Prasetya \& Sapwarobol 2018 & $3-0.371$ & $0-194$ & 0.037 & -0.750 & 0.009 & -1.915 & 0.056 \\
\hline A Hourani of al 2009 & -0.259 & $0-133$ & 0.018 & -0.520 & 0.001 & -1.952 & 0.051 \\
\hline Ziaee et al. 2006 & 0.117 & $0-110$ & 0.012 & -0.099 & 0.334 & 1.061 & 0.288 \\
\hline Mahboob et al 1999 & -0.322 & $0-170$ & 0.029 & -0.654 & 0.011 & -1.895 & 0.058 \\
\hline Unalacak et al 2011 & -0.473 & 0.227 & 0.052 & -0.919 & -0.028 & -2.082 & 0.037 \\
\hline Abdulkareem et al 2017 & 0.591 & 0.294 & 0.087 & 0.014 & 1.168 & 2.008 & 0.045 \\
\hline Feizollahzadeh et al 2014 & 0.406 & $0-123$ & 0.015 & 0.165 & 0.648 & 3.301 & 0.001 \\
\hline Afrasiabi et al 2003 & -0.618 & 0.261 & 0.068 & $-1 \cdot 129$ & -0.106 & $-2 \cdot 364$ & 0.018 \\
\hline Shehab et al 2012 & 0.147 & $0-128$ & 0.016 & $-0-104$ & 0.398 & 1.146 & 0.252 \\
\hline Celik et al 2014 & -0.116 & 0.152 & 0.023 & -0.414 & 0.182 & -0.765 & 0.444 \\
\hline Oukerro et al 2013 & 0.432 & $0-166$ & 0.028 & 0.105 & 0.758 & 2.593 & 0.010 \\
\hline El Ati et al 1995 & -0.483 & 0.252 & 0.064 & -0.977 & 0.012 & -1.914 & 0.056 \\
\hline Mansi \& Amneh 2007 & -0.293 & $0-155$ & 0.024 & -0.596 & 0.010 & -1.893 & 0.058 \\
\hline Ramadan 2002 & -0.534 & 0.255 & 0.065 & -1.034 & -0.033 & -2.090 & 0.037 \\
\hline Pirsaheb et al 2013 & -0.271 & 0.082 & 0.007 & -0.432 & -0.110 & -3.296 & 0.001 \\
\hline Ongsara et al 2017 & 0.235 & 0.124 & 0.015 & -0.009 & 0.478 & 1.888 & 0.059 \\
\hline Kassab et al 2004 & 0.280 & $0-148$ & 0.022 & -0.010 & 0.570 & 1.892 & 0.059 \\
\hline Bahiji et al 2013 & 0.763 & 0.231 & 0.053 & 0.311 & 1.216 & 3.310 & 0.001 \\
\hline Kassab ef al 2003 & 0.523 & $0-158$ & 0.025 & 0.213 & 0.833 & 3.305 & 0.001 \\
\hline Saleh et al 2005 & 0.244 & $0-129$ & 0.017 & -0.009 & 0.498 & 1.889 & 0.059 \\
\hline Hosseini \& Hejazi 2015 & 0.725 & 0.219 & 0.048 & 0.296 & 1.155 & $3 \cdot 310$ & 0.001 \\
\hline Adlouni et al 1997 & -0.627 & 0.189 & 0.036 & -0.998 & -0.255 & $-3 \cdot 308$ & 0.001 \\
\hline Esmaeilzadeh \& Borne 2016 & 0.518 & 0.270 & 0.073 & -0.011 & 1.047 & 1.920 & 0.055 \\
\hline Fakhrzadeh et al 2003 & -0.217 & 0.105 & 0.011 & -0.423 & -0.010 & -2.060 & 0.039 \\
\hline Lamine et al 2006 & -0.348 & $0-183$ & 0.034 & -0.707 & 0.011 & -1.898 & 0.058 \\
\hline Bilto 1998 & -0.220 & 0.116 & 0.014 & $-0-448$ & 0.008 & -1.887 & 0.059 \\
\hline Ibrahim et al 2008 & -0.543 & 0.272 & 0.074 & -1.076 & -0.011 & -2.000 & 0.045 \\
\hline Khan et al 2017 & -0.322 & 0.170 & 0.029 & -0.654 & 0.011 & -1.895 & 0.058 \\
\hline AlNahari \& Kouja 201 & 0.392 & 0.198 & 0.039 & 0.004 & 0.779 & 1.980 & 0.048 \\
\hline Senhadji et al 2009 & 0.280 & $0-148$ & 0.022 & -0.010 & 0.570 & 1.892 & 0.059 \\
\hline Rahbar et al 2019 & -0.605 & $0-183$ & 0.033 & -0.964 & -0.247 & -3.308 & 0.001 \\
\hline Bakki et al 2017 & 0.138 & 0.115 & 0.013 & -0.087 & 0.363 & 1.204 & 0.228 \\
\hline Rabiee ef al 2012 & 0.297 & 0.144 & 0.021 & 0.015 & 0.579 & 2.065 & 0.039 \\
\hline Naeeme Ganjali et al 2016 & -0.310 & 0.150 & 0.023 & -0.604 & -0.016 & -2.066 & 0.039 \\
\hline McNeil et al 2014 & 0.429 & 0.225 & 0.051 & -0.012 & 0.871 & 1.907 & 0.057 \\
\hline Pathan \& Patil 2010 & -0.651 & $0-197$ & 0.039 & -1.036 & -0.265 & -3.309 & 0001 \\
\hline Aksungar et al 2005 & 0.390 & 0.205 & 0.042 & -0.012 & 0.793 & 1.902 & 0057 \\
\hline Nachvak et al 2018 & -0.164 & 0.081 & 0.007 & $-0-323$ & -0.005 & -2.017 & 0.044 \\
\hline & -0.088 & 0.042 & 0.002 & -0.171 & -0.004 & -2.066 & 0.039 \\
\hline
\end{tabular}

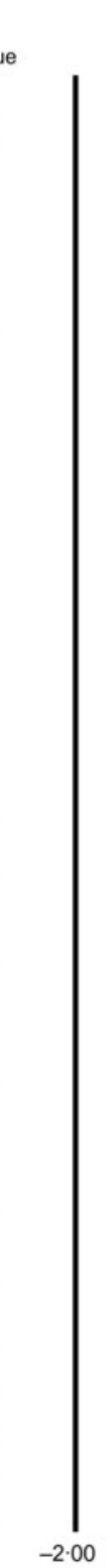

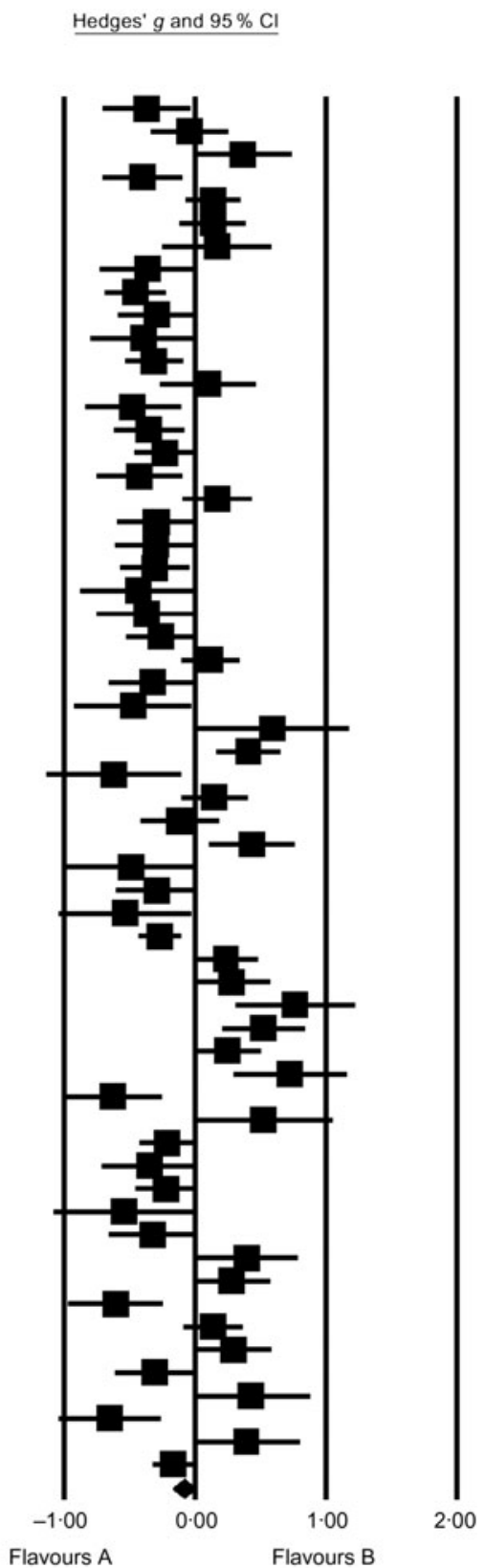

Fig. 4. According to Hedges' g value with $95 \% \mathrm{Cl}$, small (-0.088) significant reduction in serum TAG was induced by Ramadan fasting. Heterogeneity statistics: $95 \% \mathrm{Cl}$ $-0.171,-0.004, I^{2}=78 \%$. Hedges' $g$ value is considered small when value $=0.2$, medium $=0.5$, large $=0.8$.

\section{HDL-cholesterol}

Age (online Supplementary Fig. S18) and fasting time/d (online Supplementary Fig. S19) were NS moderators for RDIF-induced changes in HDL. However, sex was significant in explaining the variation in $\operatorname{HDL}(\beta=0 \cdot 15, P=0 \cdot 005$; online Supplementary Fig. S20), which suggested that men experienced larger changes in HDL than women during Ramadan month. Five countries contributed three or more studies that measured HDL changes during Ramadan month (online Supplementary Fig. S21): Iran $(K=19, N$ 1165, Hedges' $g=0 \cdot 135,95 \%$ CI $\left.0 \cdot 002,0 \cdot 268, I^{2}=79 \cdot 4 \%\right)$, Turkey $(K=6$, $N$ 210, Hedges' $g=0 \cdot 244,95 \%$ CI $\left.-0.087,0.574, I^{2}=80 \cdot 4 \%\right)$,
Jordan $(K=4, N$ 243, Hedges' $g=0 \cdot 137,95 \%$ CI $-0 \cdot 124$, $\left.0 \cdot 397, I^{2}=76 \cdot 1 \%\right)$, Pakistan $(K=3, N 175$, Hedges' $g=-0 \cdot 142$, $95 \%$ CI $\left.-0.411,0 \cdot 126, I^{2}=67 \cdot 6 \%\right)$ and Saudi Arabia $(K=3$, $N 94$, Hedges' $g=-0.019,95 \%$ CI $\left.-0.655,0 \cdot 617, I^{2}=88.5 \%\right)$.

\section{Systolic blood pressure}

Age, sex and fasting time had no significant impact on SBP changes during RDIF (online Supplementary Figs. S22-S24). Two countries contributed three or more studies that measured SBP changes during RDIF (online Supplementary Fig. S25): Iran $(K=7, N$ 522, Hedges' $g=-0.226,95 \%$ CI -0.313 , 


\begin{tabular}{|c|c|c|c|c|c|c|c|}
\hline \multirow[t]{2}{*}{ Study name } & \multicolumn{7}{|c|}{ Statistics for each study } \\
\hline & $\begin{array}{c}\text { Hedges' } \\
g\end{array}$ & $\begin{array}{l}\text { Standard } \\
\text { error }\end{array}$ & Variance & $e$ limit & $\begin{array}{c}\text { Upper } \\
\text { limit }\end{array}$ & Z-Value & $P$-Value \\
\hline Babaei et al. 2016 & -0.183 & 0.164 & 0.027 & -0.505 & 0.140 & -1.111 & 0.266 \\
\hline Akaberi et al. 2014 & 0.530 & 0.160 & 0.026 & 0.216 & 0.844 & 3.305 & 0.001 \\
\hline Sülū et al. 2008 & 0.517 & 0.156 & 0.024 & 0.210 & 0.823 & 3.305 & 0.001 \\
\hline Sijavandi et al. 2015 & -0.253 & 0.107 & 0.011 & -0.463 & -0.044 & $-2 \cdot 372$ & 0.018 \\
\hline Abubakar et al. 2018 & 0.118 & 0.128 & 0.016 & -0.133 & 0.368 & 0.919 & 0.358 \\
\hline Farshidfar et al. 2006 & 0.521 & 0.225 & 0.051 & 0.081 & 0.962 & $2 \cdot 319$ & 0.020 \\
\hline Mohammadzade et al. 2011 & 0.651 & 0.197 & 0.039 & 0.265 & 1.036 & 3.309 & 0.001 \\
\hline Kiyani et al.2015 & -0.226 & 0.112 & 0.013 & -0.445 & -0.006 & -2.011 & 0.044 \\
\hline Suriani et al. 2015 & -0.369 & 0.112 & 0.012 & -0.588 & -0.150 & -3.299 & 0.001 \\
\hline Akhtaruzzaman et al. 2014 & 0.403 & 0.191 & 0.037 & 0.028 & 0.778 & $2 \cdot 106$ & 0.035 \\
\hline Ismail \& Haron 2014 & 0.452 & 0.184 & 0.034 & 0.091 & 0.813 & 2.452 & 0.014 \\
\hline Mohammed 2011 & 0.264 & 0.134 & 0.018 & 0.001 & 0.527 & 1.969 & 0.049 \\
\hline Mansi 2007 & 0.247 & 0.120 & 0.014 & 0.012 & 0.483 & 2.061 & 0.039 \\
\hline Furuncuoglu et al. 2007 & -0.304 & 0.161 & 0.026 & -0.619 & 0.011 & -1.894 & 0.058 \\
\hline Shariatpanahi et al. 2007 & 0.400 & 0.138 & 0.019 & 0.129 & 0.671 & 2.890 & 0.004 \\
\hline Al-Numair 2006 & 0.283 & 0.150 & 0.022 & -0.010 & 0.576 & 1.892 & 0.059 \\
\hline Aksungar et al. 2007 & 0.552 & 0.167 & 0.028 & 0.225 & 0.879 & 3.306 & 0.001 \\
\hline Mohajeri et al. 2013 & -0.125 & 0.130 & 0.017 & -0.380 & 0.130 & -0.958 & 0.338 \\
\hline Begum et al. 2015 & 0.441 & 0.134 & 0.018 & 0.179 & 0.703 & 3.302 & 0.001 \\
\hline Rahman et al. 2004 & 0.449 & 0.226 & 0.051 & 0.006 & 0.892 & 1.987 & 0.047 \\
\hline Prasetya \& Sapwarobol 2018 & -0.693 & 0.209 & 0.044 & -1.103 & -0.282 & -3.309 & 0.001 \\
\hline Al Hourani ot al 2009 & 0.239 & 0.133 & 0.018 & -0.021 & 0.499 & 1.801 & 0.072 \\
\hline Ziaee et al. 2006 & -0.376 & 0.114 & 0.013 & -0.599 & -0.153 & $-3 \cdot 300$ & 0.001 \\
\hline Mahboob et al. 1999 & 0.322 & 0.170 & 0.029 & -0.011 & 0.654 & 1.895 & 0.058 \\
\hline AbdulKareem et al. 2017 & 0.591 & 0.294 & 0.087 & 0.014 & 1.168 & 2.008 & 0.045 \\
\hline deh et al. 2014 & 0.026 & 0.118 & 0.014 & -0.206 & 0.258 & 0.222 & 0.824 \\
\hline Afras & 0 . & 2 & 4 & -0 & 77 & & 56 \\
\hline Shehab et al. 2012 & 0.268 & 0.130 & 0.017 & 0.013 & 0.522 & 2.063 & 0.039 \\
\hline Celik et al. 2014 & 0.050 & 0.152 & 0.023 & -0.247 & 0.347 & 0.329 & 0.742 \\
\hline Akanji et al. 2000 & -0.271 & 0.143 & 0.021 & -0.552 & 0.010 & -1.891 & 0.059 \\
\hline Oukerro et al. 2013 & -0.308 & 0.163 & 0.027 & -0.627 & 0.011 & -1.894 & 0.058 \\
\hline Mansi \& Amneh 2007 & 0.321 & 0.155 & 0.024 & 0.017 & 0.626 & 2.067 & 0.039 \\
\hline Pirsaheb et al. 2013 & 0.128 & 31 & 0.007 & -0.031 & 0.287 & 83 & 0.113 \\
\hline Ongsara et al. 2017 & 0.235 & 0.124 & 0.015 & -0.009 & 0.478 & 1.888 & 0.059 \\
\hline Bahijri et al. 2013 & -0.763 & 0.231 & 0.053 & -1.216 & -0.311 & $-3 \cdot 310$ & 0.001 \\
\hline Saleh et al.2005 & 0.2 & 29 & 0.017 & -0.009 & 0.498 & 1.889 & 0.059 \\
\hline Hosseini \& Hejazi 2015 & -0.382 & 0.201 & 0.040 & -0.776 & 0.012 & -1.901 & 0.057 \\
\hline Adlouni et al. 1997 & 0.627 & 9 & 36 & 55 & 0.998 & 3.308 & 0.001 \\
\hline Esmaeilzadeh \& Borne 2016 & -0.518 & 0.270 & 0.073 & -1.047 & 0.011 & -1.920 & 0.055 \\
\hline Maislos et al. 1998 & 0.785 & 0.237 & 0.056 & 0.320 & 1.250 & $3 \cdot 310$ & 0.001 \\
\hline Fakhrzadeh et al. 2003 & 0.217 & 0.105 & 0.011 & 0.010 & 0.423 & 2.060 & 0.039 \\
\hline Lamine et al. 2006 & 0.364 & 0.184 & 0.034 & 0.003 & 0.724 & 1.977 & 0.048 \\
\hline Bilto 1998 & -0.229 & 0.117 & 0.014 & -0.458 & -0.001 & -1.967 & 0.049 \\
\hline Qujeq et al. 2002 & 0.227 & 0.110 & 0.012 & 0.011 & 0.443 & 2.060 & 0.039 \\
\hline Khan et al. 2017 & -0.353 & 0.171 & 0.029 & -0.687 & -0.019 & -2.069 & 0.039 \\
\hline AINahari \& Kouja 2014 & 0.375 & 0.197 & 0.039 & -0.012 & 0.761 & 1.900 & 0.057 \\
\hline Senhadji et al. 2009 & 0.280 & 0.148 & 0.022 & -0.010 & 0.570 & 1.892 & 0.059 \\
\hline Rahbar et al. 2019 & 0.562 & 0.181 & 0.033 & 0.208 & 0.917 & 3.109 & 0.002 \\
\hline Bakki et al. 2017 & 0.392 & 0.119 & 0.014 & 0.159 & 0.624 & 3.300 & 0.001 \\
\hline Rabiee et al. 2012 & -0.297 & 0.144 & 0.021 & -0.579 & -0.015 & -2.065 & 0.039 \\
\hline Naeeme Ganjali et al.2016 & 0.310 & 0.150 & 0.023 & 0.016 & 0.604 & 2.066 & 0.039 \\
\hline McNeil et al. 2014 & 0.429 & 0.225 & 0.051 & -0.012 & 0.871 & 1.907 & 0.057 \\
\hline Pathan \& Patil 2010 & 0.490 & 0.189 & 0.036 & 0.120 & 0.860 & 2.597 & 0.009 \\
\hline Aksungar et al. 2005 & 0.430 & 0.207 & 0.043 & 0.024 & 0.835 & 2.077 & 0.038 \\
\hline Nachvak et al. 2018 & 0.174 & 0.081 & 0.007 & 0.014 & 0.333 & $2 \cdot 136$ & 0.033 \\
\hline & & 14 & 002 & .064 & 0.236 & 3.419 & 0.001 \\
\hline
\end{tabular}


Table 3. Overall Hedges' $g$ values for the metabolic syndrome components and statistical values for the three moderators (age, sex and fasting time) at the end of Ramadan

\begin{tabular}{|c|c|c|c|c|c|c|c|c|c|}
\hline \multirow[b]{2}{*}{ Component } & \multirow[b]{2}{*}{$K^{*}$} & \multirow[b]{2}{*}{$N \dagger$} & \multirow[b]{2}{*}{$I^{2} \ddagger$} & \multirow[b]{2}{*}{$\tau^{2} \S$} & \multirow[b]{2}{*}{$\begin{array}{l}\text { Overall } \\
\text { Hedges' } g\end{array}$} & \multirow[b]{2}{*}{$95 \% \mathrm{Cl}$} & \multicolumn{3}{|c|}{ Moderators } \\
\hline & & & & & & & Age & Sex (\% Male) & $\begin{array}{l}\text { Fasting } \\
\text { time/d }\end{array}$ \\
\hline WC & 24 & 1557 & $49 \%$ & $P=0.001$ & -0.32 & $-0.39,-0.24$ & $\begin{array}{c}\beta=-0.002 \\
P=0.78\end{array}$ & $\begin{array}{c}\beta=-0.20 \\
P=0.03\end{array}$ & $\begin{array}{c}\beta=-0.001 \\
P=0.08\end{array}$ \\
\hline FG & 51 & 2318 & $26.6 \%$ & $P=0.001$ & $-0 \cdot 10$ & $-0.20,0.004$ & $\begin{array}{l}\beta=0.005 \\
P=0.05\end{array}$ & $\begin{array}{c}\beta=-0.10 \\
P=0.08\end{array}$ & $\begin{array}{c}\beta=-0.001 \\
P=0.001\end{array}$ \\
\hline TAG & 63 & 2862 & $78 \%$ & $P=0.04$ & -0.10 & $-0.12,0.004$ & $\begin{array}{l}\beta=0.0004 \\
P=0.90\end{array}$ & $\begin{array}{c}\beta=-0.14 \\
P=0.01\end{array}$ & $\begin{array}{c}\beta=-0.005 \\
P=0.01\end{array}$ \\
\hline HDL & 57 & 2771 & $79 \%$ & $P=0.001$ & 0.15 & $0.07,0.24$ & $\begin{array}{c}\beta=-0.0003 \\
P=0.90\end{array}$ & $\begin{array}{l}\beta=0.15 \\
P=0.005\end{array}$ & $\begin{array}{c}\beta=-0.002 \\
P=0.25\end{array}$ \\
\hline SBP & 22 & 1172 & $78 \%$ & $P=0.001$ & -0.25 & $-0.38,-0.11$ & $\begin{array}{c}\beta=-0.015 \\
P=0.064\end{array}$ & $\begin{array}{c}\beta=-0.216 \\
P=0.20\end{array}$ & $\begin{array}{l}\beta=0.00005, \\
P=0.96\end{array}$ \\
\hline
\end{tabular}

FG, fasting plasma glucose; SBP, systolic blood pressure; WC, waist circumference.

${ }^{*} K$ : denotes number of studies.

$+N$ : denotes number of participants.

$\ddagger I^{2}$ statistic describes the percentage of variation across studies due to heterogeneity rather than chance ${ }^{(108)}$.

$\S$ In a random-effects meta-analysis, the extent of variation among the effects observed in different studies (between-study variance) is referred to as $\tau^{2(109)}$

Study name

Aksubheen et al. 2017

Al-Barha \& Aljaloud 2018

Dewanti et al. 2006

Esmaeilzadeh \& Borne 2016

Fakhrzadeh et al. 2003

Faris et al. 2012

Khan et al. 2017

Malekmakan et al. 2017

Mansi 2007

Mansi \& Amneh 2007

Mohammadzade et al. 2011

Narouzy et al. 2017

Ongsara et al. 2017

Pirsaheb et al. 2013

Rahman et al. 2004

Sezen et al. 2016

Shariatpanahi et al. 2007

Shehab et al. 2012

Sijavandi et al. 2015

Suriani et al. 2015

Unalacak et al. 2011

\begin{tabular}{|c|c|c|c|c|c|c|}
\hline & & set & or each & & & \\
\hline $\begin{array}{c}\text { Hedges' } \\
g\end{array}$ & $\begin{array}{c}\text { Standard } \\
\text { error }\end{array}$ & Variance & $\begin{array}{l}\text { Lower } \\
\text { limit }\end{array}$ & $\begin{array}{l}\text { Upper } \\
\text { limit }\end{array}$ & Z-Value & $P$-Value \\
\hline-0.737 & 0.348 & 0.121 & -1.419 & -0.056 & $-2 \cdot 121$ & 0.034 \\
\hline 0.286 & 0.151 & 0.023 & -0.010 & 0.582 & 1.892 & 0.058 \\
\hline-0.577 & 0.174 & 0.030 & -0.918 & -0.235 & -3.307 & 0.001 \\
\hline-1.062 & 0.322 & 0.104 & -1.692 & -0.431 & -3.300 & 0.001 \\
\hline-0.198 & 0.105 & 0.011 & -0.404 & 0.008 & -1.886 & 0.059 \\
\hline-0.487 & 0.148 & 0.022 & -0.777 & -0.198 & -3.304 & 0.001 \\
\hline 0.419 & 0.173 & 0.030 & 0.081 & 0.758 & 2.426 & 0.015 \\
\hline-0.213 & 0.104 & 0.011 & -0.417 & -0.009 & -2.049 & 0.040 \\
\hline-0.247 & 0.120 & 0.014 & -0.483 & -0.012 & -2.061 & 0.039 \\
\hline-0.321 & 0.155 & 0.024 & -0.626 & -0.017 & -2.067 & 0.039 \\
\hline-0.348 & 0.183 & 0.034 & -0.707 & 0.011 & -1.898 & 0.058 \\
\hline-0.361 & 0.278 & 0.078 & -0.907 & 0.185 & -1.296 & 0.195 \\
\hline 0.423 & 0.128 & 0.016 & 0.172 & 0.674 & 3.301 & 0.001 \\
\hline-0.211 & 0.082 & 0.007 & -0.370 & -0.051 & -2.580 & 0.010 \\
\hline-0.834 & 0.252 & 0.063 & -1.327 & -0.340 & -3.309 & 0.001 \\
\hline 0.101 & 0.119 & 0.014 & -0.132 & 0.333 & 0.850 & 0.395 \\
\hline-0.463 & 0.140 & 0.020 & -0.737 & -0.188 & -3.303 & 0.001 \\
\hline-0.305 & 0.130 & 0.017 & -0.560 & -0.049 & -2.336 & 0.019 \\
\hline-0.087 & 0.105 & 0.011 & -0.293 & 0.120 & -0.823 & 0.411 \\
\hline-0.133 & 0.109 & 0.012 & -0.346 & 0.080 & -1.225 & 0.221 \\
\hline-0.834 & 0.252 & 0.063 & $-1 \cdot 327$ & -0.340 & -3.309 & 0.001 \\
\hline-0.239 & 0.068 & 0.005 & -0.372 & -0.106 & -3.529 & 0.000 \\
\hline
\end{tabular}

$-2 \cdot 00$
Hedges' $g$ and $95 \% \mathrm{Cl}$

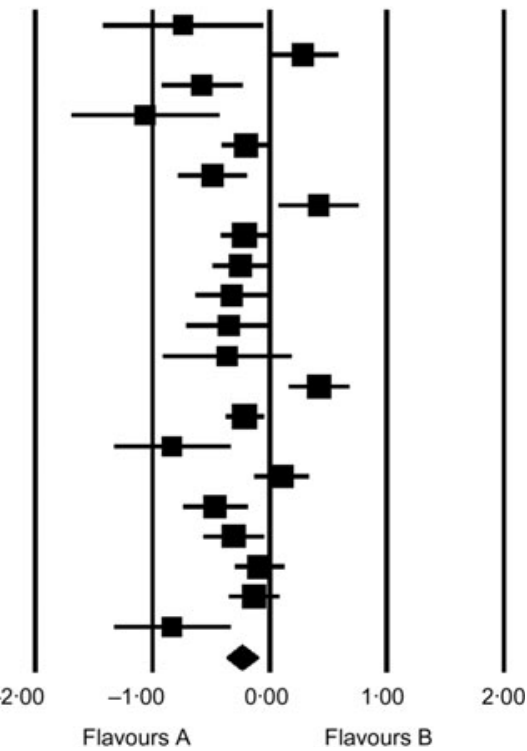

Fig. 6. According to Hedges' $g$ value with $95 \% \mathrm{Cl}$, small $(-0.239)$ significant reduction in systolic blood pressure was induced by Ramadan fasting. Heterogeneity statistics: $95 \% \mathrm{Cl}-0.372,-0.106, l^{2}=78 \%$. Hedges' $g$ value is considered small when value $=0.2$, medium $=0.5$, large $=0.8$.

days were reduced. Further, it is expected that differences may be existing in lipid profile changes between pre- and postmenopause women included in the present analysis, as supported by the published literature ${ }^{(111,112)}$. However, such differences cannot be inferred from the present analysis and need to be executed in a special sub-group analysis.

The RDIF model is a widely known and well-studied model of religion-based intermittent fasting. Intermittent fasting is associated with improved human health ${ }^{(113-116)}$. The results of this meta-analysis expanded existing knowledge and confirmed that practicing RDIF had positive health impacts. RDIF had a beneficial effect on abdominal obesity, serum lipids, glucose metabolism and BP levels; all of which are the MetS components and risk factors for the development of insulin resistance, diabetes and CVD. The beneficial impact of RDIF on health is further reinforced by its ability to induce and activate antioxidant and anti-inflammatory mechanisms ${ }^{(8,18)}$

The small reduction in WC shown in our results was consistent with (and may partially explain) the significant small RDIF-induced reduction in inflammatory markers including IL-6, TNF- $\alpha$ and $h s$-C-reactive protein and the oxidative stress marker malondialdehyde shown in a recent meta-analysis ${ }^{(8)}$. 
Table 4. Characteristics of studies included in each of the metabolic syndrome components reviewed and analysed by countries with three or more studies

\begin{tabular}{|c|c|c|c|c|c|c|}
\hline Component & Country & $K^{*}$ & $N+$ & $I^{2} \ddagger$ & Hedges' $g$ & $95 \% \mathrm{Cl}$ \\
\hline WC & Iran & 7 & 783 & 0.000 & -0.275 & $-0.348,0.002$ \\
\hline \multirow[t]{6}{*}{$\mathrm{FG}$} & Iran & 15 & 828 & $81 \cdot 865$ & -0.173 & $-0.348,0.002$ \\
\hline & Saudi Arabia & 5 & 218 & 80.395 & 0.075 & $-0.240,0.390$ \\
\hline & Turkey & 4 & 146 & 93.036 & -0.069 & $-0.735,0.596$ \\
\hline & Tunisia & 4 & 109 & 81.565 & 0.180 & $-0.271,0.630$ \\
\hline & Pakistan & 3 & 175 & 69.743 & -0.215 & $-0.494,0.064$ \\
\hline & Jordan & 3 & 186 & 0.000 & -0.239 & $-0.383,-0.095$ \\
\hline \multirow[t]{6}{*}{ TAG } & Iran & 20 & 1156 & 78.652 & -0.073 & $-0.204,0.058$ \\
\hline & Turkey & 6 & 210 & 63.288 & -0.229 & $-0.458,0.001$ \\
\hline & Jordan & 4 & 243 & 0.000 & -0.244 & $-0.370,-0.117$ \\
\hline & Pakistan & 3 & 175 & 83.488 & -0.212 & $-0.591,0.168$ \\
\hline & Tunisia & 4 & 109 & $85 \cdot 646$ & -0.116 & $-0.706,0.473$ \\
\hline & Saudi Arabia & 3 & 94 & 88.524 & 0.269 & $-0.366,0.905$ \\
\hline \multirow[t]{5}{*}{ HDL } & Jordan & 4 & 243 & $76 \cdot 175$ & 0.137 & $-0.124,0.397$ \\
\hline & Iran & 19 & 1165 & $79 \cdot 422$ & 0.135 & $0.002,0.268$ \\
\hline & Turkey & 5 & 210 & 80.481 & 0.244 & $-0.087,0.574$ \\
\hline & Pakistan & 3 & 175 & $67 \cdot 711$ & -0.142 & $-0.411,0.126$ \\
\hline & Saudi Arabia & 3 & 94 & 88.510 & -0.019 & $-0.655,0.617$ \\
\hline \multirow[t]{2}{*}{ SBP } & Iran & 7 & 522 & 0.000 & -0.226 & $-0.313,-0.138$ \\
\hline & Jordan & 3 & 162 & 0.000 & -0.342 & $-0.501,-0.183$ \\
\hline
\end{tabular}

FG, fasting plasma glucose; SBP, systolic blood pressure; WC, waist circumference.

${ }^{*} K$ : denotes number of studies.

$\dagger N$ : denotes number of participants.

$\ddagger I^{2}$ statistic describes the percentage of variation across studies due to heterogeneity rather than chance ${ }^{(108)}$.

The presence of the MetS has also been associated with lower plasma adiponectin levels ${ }^{(117)}$, indicating adipose tissue dysfunction and a two- to four-times increased risk for developing CVD and type 2 diabetes ${ }^{(118)}$. Similarly, several reports have indicated RDIF was associated with variable increments in adiponectin levels in fasting people $e^{(119-121)}$. Recently, we found that RDIF was associated with significant reduction in visceral fat surface area (measured by MRI) in fifty-seven overweight/obese participants, concomitant with significant reductions proinflammatory cytokines (IL- 6 and TNF- $\alpha$ ), and a significant increase in the anti-inflammatory cytokine IL- $10^{(122)}$. In addition, Fernando et al. conducted a systematic review and meta-analysis on the impact of RDIF on body fatness. They found a significant reduction in fat percentage between pre- and post-Ramadan in people with overweight or obesity $(-1 \cdot 46,95 \%$ CI -2.57 , $-0.35 \%, \quad P=0.010)$ compared with fasting subjects with normal weight ${ }^{(9)}$. This implied that RDIF incurs a pronounced protective effect against the MetS in people at risk for obesity.

In the present meta-analysis, there was a small but significant RDIF-induced reduction in serum TAG in healthy people, which may contribute to lowering the risk for atherogenesis. This plausible effect is supported by other reports that showed an anti-RDIF-induced atherogenic effect in ameliorating LDLcholesterol and apo B, and improving anti-atherogenic apo A levels in subjects with normal weight ${ }^{(107,123,124)}$ and a small significant increase in HDL levels at the end of Ramadan. Other anti-atherogenic impacts for RDIF include: significant improvements in blood coagulation parameters ${ }^{(125)}$, along with significant reductions in total cholesterol ${ }^{(84,85,95,107)}$, very LDL $^{(57,58,61,125)}$, LDL:HDL and cholesterol:HDL ratios $^{(123,126)}$ and atherogenic index ((total cholesterol - HDL-cholesterol)/HDLcholesterol) $^{(43)}$.
RDIF was also shown to improve endothelial function ${ }^{(127)}$ by increasing nitric oxide production (important for normal endothelium) ${ }^{(128)}$ and improving the heat shock protein HSP70, which has been shown to possess atheroprotective and endothelial-improving effects ${ }^{(126)}$. Interestingly, the RDIFinduced cardioprotective effect extended for about 1 month after Ramadan month cessation ${ }^{(27,124)}$. This indicated that RDIF has an annual short-term transient protective function against developing CVD. Finally, the small but significant reductions in the inflammatory ( $h s$-C-reactive protein, TNF- $\alpha$ and IL-6) and oxidative stress (malondialdehyde) markers induced by RDIF reported in a recent meta-analysis ${ }^{(8)}$ support the cardioprotective effect of Ramadan fasting. These markers have been shown to be involved in the etiopathogenesis of atherosclerosis and other $\mathrm{CVD}^{(129-131)}$. The potential for RDIF to improve antioxidants was supported by our recent findings that the relative gene expressions in obese subjects were significantly increased for three antioxidant genes (superoxide dismutase, SOD2; mitochondrial transcription factor A, TFAM; and nuclear factor erythroid 2-related factor 2, Nrf2) at the end of Ramadan, with percent increases of $90.5 \%, 54.1 \%$ and $411.5 \%$ for these genes, respectively ${ }^{(132)}$. However, the protective effect of RDIF against CVD does not appear to be substantiated by rigorous scientific evidence available to date. This suggests that more controlled research is warranted.

A decreased level of HDL-cholesterol is a basic component in the diagnosis and definition of the $\mathrm{MetS}^{(2)}$. In epidemiological studies, this abnormality has been closely associated with a higher risk for atherosclerotic CVD. Despite debate as to whether low HDL is involved in the pathogenesis of CVD, there is agreement that low HDL-cholesterol is a marker for increased risk for $\mathrm{CVD}^{(2,133)}$. In the present review, the 
presence of small but significant increments in HDL at the end of RDIF was an added RDIF-induced protective factor against CVD, as dyslipidemia and its cumulative metabolic derangements are all involved in the etiopathogenesis of the MetS and its complications.

Several mechanisms have been suggested to explain the relationship between hypertension and the MetS. These include the release of angiotensinogen from adipose tissue, expansion of intravascular volume, enhanced renal reabsorption of sodium (possibly due to insulin resistance), activations of the renin-angiotensin-aldosterone system and sympathetic nervous system and insulin resistance. Current consensus is that these factors act in conjunction to raise $\mathrm{BP}^{(2)}$. Consistent with the reduction in SBP, several reports indicated there was reduction in diastolic BP after $\operatorname{RDIF}^{(8,92,100,102)}$

Insulin resistance with fatty acid flux is an accepted hypothesised mechanism for the underlying pathophysiology of the MetS, with low-grade chronic inflammation and oxidative stress being mechanisms that accompany the MetS ${ }^{(134)}$. A recent systematic review and meta-analysis revealed RDIF had a positive effect on lowering inflammatory and oxidative stress markers ${ }^{(8)}$, with emphasis on IL- 6 and TNF- $\alpha$ as the pro-inflammatory markers most involved in the pathogenesis of the $\mathrm{MetS}^{(134)}$. Several reports indicated RDIF was associated with variable changes in serum insulin and insulin resistance (presented as homeostatic model assessment of insulin resistance levels). Although several reports indicated a lack of significant changes in serum insulin and insulin resistance during $\operatorname{RDIF}^{(37,78,135,136)}$, other studies reported significant changes during $\operatorname{RDIF}^{(67-69,106)}$. However, considerable variations in insulin and insulin resistance measurements imply that behavioural, dietary, and lifestyle factors affect these measurements, including quantity and quality of foods consumed, duration of fasting time, body weight and health status before fasting, differences in physical activity levels and differences in circadian rhythm and hormonal changes during RDIF.

The heterogeneity of the studies included in this metaanalysis regarding the MetS components could be attributed to various effects and confounding factors and perhaps explained by inconsistencies in the designs, procedures and interpretation of results of studies conducted during Ramadan. It is believed that a critical violation performed by many fasting people during Ramadan is skipping the predawn meal (suboor), which may contribute to a significant daily energetic deficit and is likely to promote metabolic derangements, increased postprandial insulin levels and fat oxidation and induce confounding in the results ${ }^{(53)}$

Assuming that Ramadan fasting represents a form of time-restricted feeding as reported by Patterson \& Sears ${ }^{(114)}$, our findings were consistent with other research on human and animal intermittent energy restriction and time-restricted feeding. There is a growing evidence base demonstrating short-to-medium term benefits of time-restricted feeding on glucose and lipid homeostasis, even in the absence of significant total daily energetic restriction (reduction in $25-40 \%$ of total daily energetic intake). The majority of published research conducted during Ramadan revealed a lack of significant changes in total daily energetic intake during Ramadan compared with pre-fasting energetic intake ${ }^{(18,97,137,138)}$. By combining studies on RDIF, the present meta-analysis analysed a large sample, thereby increasing the power of the studies in showing the effects of RDIF. However, this meta-analysis had some limitations. In most included studies that evaluated the impact of RDIF on body weight, lipids and other metabolic profiles, there was no information as to whether participants consumed a predawn breakfast. During RDIF, subjects are at postprandial state if blood parameters are measured in the morning because of the predawn meal (suboor). Several studies focused on RDIF did not report whether baseline laboratory parameters measured after an overnight fast before the initiation of Ramadan fasting were compared with laboratory parameters measured a couple of hours before the fast was broken at sunset, which would be the equivalent of an overnight fast. More importantly, the assessment of several key biomarkers of glucose and lipid metabolism and various hormones (including leptin, melatonin, ghrelin, cortisol and adiponectin) exhibits circadian rhythm and requires 24-h blood monitoring with multiple time points ${ }^{(139)}$. Measurement of these parameters at only one or a few time points may to lead to biased outcomes and potentially provide false data on increased/decreased levels, depending on the measurement time.

Dawn-to-sunset Ramadan fasting starts at dawn and ends at sunset. Therefore, fasting people have two major meals a day: breakfast before dawn and dinner after sunset, and may eat ad libitum from sunset until dawn. In general, it is a common practice for Ramadan fasters to work and fast during the daytime, have dinner, sleep and wake $1 \mathrm{~h}$ before dawn to eat and restart fasting at dawn. Most studies on RDIF did not provide explicit dietary information about the frequency of meals between sunset and dawn, meal content during night hours and sleeping times $^{(97,140)}$. Furthermore, some study subjects may secretly break their fast and further confound the desired adaptive response. No information on compliance monitoring with fasting was available for any of the included studies. Well-designed clinical trials that evaluate the impact of dawn-to-sunset fasting on BMI and key metabolic parameters before, during and after fasting would provide important information about health maintenance ${ }^{(97)}$.

\section{Conclusions}

It can be concluded from the reviewed and analysed the MetS components that RDIF showed small reductions in components associated with increased risk for and severity of the MetS (WC, SBP, TAG and FG), with a concomitant increase in antiatherogenic HDL-cholesterol. These beneficial effects were also associated with variable improvements in other covariates involved in the etiopathogenesis factors, such as reduction in diastolic BP. The heterogeneity in the findings of the reviewed studies offers a picture of the varying dietary and lifestyle behaviours practiced during Ramadan month, along with variations in the duration of fasting and climatic and geographical conditions surrounding fasting people in different countries. 


\section{Ethical statement}

This article does not contain any studies with human participants performed by any of the authors. For this type of study formal consent was not required

\section{Acknowledgements}

Authors express their thanks to Ms Faiza Kalam for assistance in article collection and data tabulation.

The present work did not receive any form of funding from any institution or funding body.

M. E. F. and H. A. J. contributed to the conception and design of the work. M. E. F. and A. A. O. participated in searching for and collecting articles. M. E. F., J. A. and A. A. O. participated in article reviews; J. A. principally participated in data extraction. H. A. J. performed all data analyses. M. E. F. and H. A. J. contributed to drafting the paper, critically revising the paper and providing intellectual contributions to strengthen the paper. All authors were involved in writing this paper and approved the final version for publication.

The authors have no conflicts of interest to declare.

\section{Supplementary materials}

To view supplementary material for this article, please visit https://doi.org/10.1017/S000711451900254X

\section{References}

1. Grundy SM (2007) Metabolic syndrome: a multiplex cardiovascular risk factor. J Clin Endocrinol Metab 92, 399-404.

2. Grundy SM (2016) Metabolic syndrome update. Trends Cardiovasc Med 26, 364-373.

3. Sundfør T \& Svendsen M (2018) Effect of intermittent versus continuous energy restriction on weight loss, maintenance and cardiometabolic risk: a randomized 1-year trial. Nutr Metab Cardiovasc Dis 28, 698-706.

4. Harvie M, Wright C, Pegington M, et al. (2013) The effect of intermittent energy and carbohydrate restriction $v$. daily energy restriction on weight loss and metabolic disease risk markers in overweight women. Br J Nutr 110, 1534-1547.

5. Dalle Grave R, Calugi S, Centis E, et al. (2010) Lifestyle modification in the management of the metabolic syndrome: achievements and challenges. Diabetes Metab Syndr Obes 3, 373-385.

6. Sakr AH (1975) Fasting in Islam. J Am Diet Assoc 67, 17-21.

7. Sadeghirad B, Motaghipisheh S, Kolahdooz F, et al. (2014) Islamic fasting and weight loss: a systematic review and meta-analysis. Public Health Nutr 17, 396-406.

8. Faris M, Jahrami HA, Obaideen AA, et al. (2019) Impact of diurnal intermittent fasting during Ramadan on inflammatory and oxidative stress markers in healthy people: systematic review and meta-analysis. J Nutr Intermed Metab 15, 18-26.

9. Fernando H, Zibellini J, Harris R, et al. (2019) Effect of Ramadan fasting on weight and body composition in healthy non-athlete adults: a systematic review and meta-analysis. Nutrients 11, 478.

10. Kul S, Sava囚 E, Öztürk ZA, et al. (2014) Does Ramadan fasting alter body weight and blood lipids and fasting blood glucose in a healthy population? A meta-analysis.J Religion Health 53, 929-942.
11. Rouhani M \& Azadbakht L (2014) Is Ramadan fasting related to health outcomes? A review on the related evidence. J Res Med Sci 19, 987-892.

12. Salim I, Al Suwaidi J, Ghadban W, et al. (2013) Impact of religious Ramadan fasting on cardiovascular disease: a systematic review of the literature. Curr Med Res Opin 29, 343-354.

13. Ziaee V, Razaei M, Ahmadinejad Z, et al. (2006) The changes of metabolic profile and weight during Ramadan fasting. Singap Med J 47, 409-14.

14. International Diabetes Federation (IDF) (2006) The IDF Consensus Worldwide Definition of the Metabolic Syndrome. https://www.idf.org/e-library/consensus-statements/ 60-idfconsensus-worldwide-definitionof-the-metabolic-syndrome. html (accessed March 2019).

15. Freemantle N \& Geddes J (1998) Understanding and interpreting systematic reviews and meta-analyses. Part 2: metaanalyses. Evid-Based Ment Health 1, 102-104.

16. Student (1989) Six reasons for conducting a meta-analysis. Pediatrics 83, 100.

17. Stroup DF, Berlin JA, Morton SC, et al. (2000) Meta-analysis of observational studies in epidemiology: a proposal for reporting. JAMA 283, 2008-2012.

18. Faris M, Kacimi S, Al-Kurd RAA, et al. (2012) Intermittent fasting during Ramadan attenuates proinflammatory cytokines and immune cells in healthy subjects. Nutr Res 32, 947-955.

19. Nachvak SM, Pasdar Y, Pirsaheb S, et al. (2018) Effects of Ramadan on food intake, glucose homeostasis, lipid profiles and body composition composition. Eur J Clin Nutr 1, 594-600.

20. Meo SA \& Hassan A (2015) Physiological changes during fasting in Ramadan. J Pak Med Assoc 65, S6-S14.

21. Hedges LV (1981) Distribution theory for Glass's estimator of effect size and related estimators. J Educ Stat 6, 107-128.

22. Borenstein M, Hedges L, Higgins J, et al. (2005) Comprehensive Meta-analysis (Vers. 2). Englewood Cliffs, NJ: Biostat: Inc.

23. Chen H, Manning AK \& Dupuis J (2012) A method of moments estimator for random effect multivariate meta-analysis. Biometrics 68, 1278-1284.

24. Duval S \& Tweedie R (2000) A nonparametric 'trim and fill' method of accounting for publication bias in meta-analysis. J Am Stat Assoc 95, 89-98.

25. Fedail SS, Murphy D, Salih S, et al. (1982) Changes in certain blood constituents during Ramadan. Am J Clin Nutr 36, 350-353.

26. Azizi F \& Rasouli H (1987) Serum glucose, bilirubin, calcium, phosphorus, protein and albumin concentrations during Ramadan. Med J Islam Rep Iran 1, 38-41.

27. El Ati J, Beji C \& Danguir J (1995) Increased fat oxidation during Ramadan fasting in healthy women: an adaptative mechanism for body-weight maintenance. Am J Clin Nutr 62, 302-307.

28. Adlouni A, Ghalim N, Benslimane A, et al. (1997) Fasting during Ramadan induces a marked increase in high-density lipoprotein cholesterol and decrease in low-density lipoprotein cholesterol. Ann Nutr Metab 41, 242-249.

29. Bilto YY (1998) Effects of Ramadan fasting on body weight and the biochemical and haematological parameters of the blood. Arab GulfJ Sci Res 16, 1-14.

30. Maislos M, Abou-Rabiah Y, Zuili I, et al. (1998) Gorging and plasma HDL-cholesterol-the Ramadan model. Eur J Clin Nutr 52, 127-130.

31. Mahboob S, Sattarivand R, Nouri M, et al. (1999) Effect of Ramadan fasting on serum lipid profiles in normal and hyperlipidemic subjects. Saudi Med J 20, 947-950. 
32. Akanji A, Mojiminiyi O \& Abdella N (2000) Beneficial changes in serum apo A-1 and its ratio to apo B and HDL in stable hyperlipidaemic subjects after Ramadan fasting in Kuwait. Eur J Clin Nutr 54, 508-513.

33. Asgary S, Aghaei F, Naderi GA, et al. (2000) Effects of Ramadan fasting on lipid peroxidation, serum lipoproteins and fasting blood sugar. Med J Islam World Acad Sci 13, 35-38.

34. Qujeq D, Bijani K, Kalavi K, et al. (2002) Effects of Ramadan fasting on serum low-density and high-density lipoproteincholesterol concentrations. Ann Saudi Med 22, 297-299.

35. Ramadan J (2002) Does fasting during Ramadan alter body composition, blood constituents and physical performance? Med Princ Pract 11, 41-46.

36. Afrasiabi A, Hassanzadeh S, Sattarivand R, et al. (2003) Effects of Ramadan fasting on serum lipid profiles on 2 hyperlipidemic groups with or without diet pattern. Saudi Med J 24, 23-26.

37. Fakhrzadeh H, Larijani B, Sanjari M, et al. (2003) Effect of Ramadan fasting on clinical and biochemical parameters in healthy adults. Ann Saudi Med 23, 223.

38. Kassab S, Abdul-Ghaffar T, Nagalla DS, et al. (2003) Serum leptin and insulin levels during chronic diurnal fasting. Asia Pac J Clin Nutr 12, 483-487.

39. Larijani B, Zahedi F, Sanjari M, et al. (2003) The effect of Ramadan fasting on fasting serum glucose in healthy adults. Med J Malays 58, 678-680.

40. Kassab S, Abdul-Ghaffar T, Nagalla DS, et al. (2004) Interactions between leptin, neuropeptide-Y and insulin with chronic diurnal fasting during Ramadan. Ann Saudi Med 24, 345.

41. Rahman M, Rashid M, Basher S, et al. (2004) Improved serum HDL cholesterol profile among Bangladeshi male students during Ramadan fasting. East Mediterr Health J 10, 131-137.

42. Yucel A, Degirmenci B, Acar M, et al. (2004) The effect of fasting month of Ramadan on the abdominal fat distribution: assessment by computed tomography. Tohoku J Exp Med 204, 179-187.

43. Aksungar FB, Eren A, Ure S, et al. (2005) Effects of intermittent fasting on serum lipid levels, coagulation status and plasma homocysteine levels. Ann Nutr Metab 49, 77-82.

44. Saleh SA, Elsharouni SA, Cherian B, et al. (2005) Effects of Ramadan fasting on waist circumference, blood pressure, lipid profile, and blood sugar on a sample of healthy Kuwaiti men and women. Malay J Nutr 11, 143-150.

45. Al-Numair N (2006) Body weight and some biochemical changes associated with Ramadan fasting in healthy Saudi men. J Med Sci 6, 112-116.

46. Dewanti L, Watanabe C \& Ohtsuka R (2006) Unexpected changes in blood pressure and hematological parameters among fasting and nonfasting workers during Ramadan in Indonesia. Eur J Clin Nutr 60, 877-881.

47. Farshidfar G, Yousfi H, Vakili M, et al. (2006) The effect of Ramadan fasting on hemoglobin, hematocrit and blood biochemical parameters. J Res Health Sci 6, 21-27.

48. Lamine F, Bouguerra R, Jabrane J, et al. (2006) Food intake and high density lipoprotein cholesterol levels changes during Ramadan fasting in healthy young subjects. La Tunisie Médicale 84, 647-650.

49. Ziaee V, Razaei M, Ahmadinejad Z, et al. (2006) The changes of metabolic profile and weight during Ramadan fasting. Singap Med J 47, 409-414.

50. Aksungar FB, Topkaya AE \& Akyildiz M (2007) Interleukin-6, C-reactive protein and biochemical parameters during prolonged intermittent fasting. Ann Nutr Metab 51, 88-95.
51. Furuncuoglu Y, Karaca E, Aras S, et al. (2007) Metabolic, biochemical and psychiatric alterations in healthy subjects during Ramadan. Pak J Nutr 6, 209-211.

52. Mansi KMS (2007) Study the effects of Ramadan fasting on the serum glucose and lipid profile among healthy Jordanian students. Am J Appl Sci 4, 565-569.

53. Mansi K \& Amneh M (2007) Impact of Ramadan fasting on metabolism and on serum levels of some hormones among healthy Jordanian students. J Med Sci 7, 755-761.

54. Salehi M \& Neghab M (2007) Effects of fasting and a medium calorie balanced diet during the holy month Ramadan on weight, BMI and some blood parameters of overweight males. PakJ Biol Sci 10, 968-971.

55. Shariatpanahi ZV, Shariatpanahi MV, Shahbazi S, et al. (2008) Effect of Ramadan fasting on some indices of insulin resistance and components of the metabolic syndrome in healthy male adults. Br J Nutr 100, 147-151.

56. Ibrahim WH, Habib HM, Jarrar AH, et al. (2008) Effect of Ramadan fasting on markers of oxidative stress and serum biochemical markers of cellular damage in healthy subjects. Ann Nutr Metab 53, 175-181.

57. Al Hourani HM, Atoum MF, Akel S, et al. (2009) Effects of Ramadan fasting on some haematological and biochemical parameters. Jordan J Biol Sci 2, 103-108.

58. Lamri-Senhadji M, El Kebir B, Belleville J, et al. (2009) Assessment of dietary consumption and time-course of changes in serum lipids and lipoproteins before, during and after Ramadan in young Algerian adults. Singap Med J 50, 288-294.

59. Sülü B, Öztuürk B, Guüven A, et al. (2010) The effect of longterm controlled fasting (the Ramadan model) on body mass index, blood biochemistry and oxidative stress factors. Turk Klin J Med Sci 30, 855-863.

60. Norouzy A, Salehi M, Arabi H, et al.(2010) Effects of Ramadan fasting on anthropometric indices. Fasting Sustainable Health Conference 2010, p. 65.

61. Barkia A, Mohamed K, Smaoui M, et al. (2011) Change of diet, plasma lipids, lipoproteins, and fatty acids during Ramadan: a controversial association of the considered Ramadan model with atherosclerosis risk. I Health Popul Nutr 29, 486-493.

62. Mohammed ZJ (2011) The influence of Ramadan fasting on some hematological and biochemical parameters in healthy adult males. Nrsing National Iraqi Specility 24, 1-12.

63. Ünalacak M, Kara IH, Baltaci D, et al. (2011) Effects of Ramadan fasting on biochemical and hematological parameters and cytokines in healthy and obese individuals. Metab Syndr Relat Disord 9, 157-161.

64. Shehab A, Abdulle A, El Issa A, et al. (2012) Favorable changes in lipid profile: the effects of fasting after Ramadan. PLOS ONE 7, e47615.

65. Sayedda K, Kamal S \& Ahmed QS (2013) Effect of Ramadan fasting on anthropometric parameters, blood pressure, creatine phosphokinase activity, serum calcium and phosphorus in healthy students of Shri Ram Murti Smarak Institute of Medical Sciences, Bareilly-UP. Natl J Physiol Pharm Pharmacol 3, 48-52.

66. Agoumi A, Martinez Martinez F \& Garcia de la Serrana HL (2013) The assessment of the follow-up of Muslim population during the period of Ramadan. J Nutr Food Sci 3, 220.

67. Bahijri S, Borai A, Ajabnoor G, et al. (2013) Relative metabolic stability, but disrupted circadian cortisol secretion during the fasting month of Ramadan. PLOS ONE 8, e60917.

68. Haouari-Oukerro F, Ben-Attia M, Kaâbachi N, et al. (2013) Ramadan fasting influences on food intake consumption, 
sleep schedule, body weight and some plasma parameters in healthy fasting volunteers. Afr J Biotechnol 12, 3327-3332.

69. Hosseini SRA, Sardar MA, Hejazi K, et al. (2013) The effect of Ramadan fasting and physical activity on body composition, serum osmolarity levels and some parameters of electrolytes in females. Int J Endocrinol Metab 11, 88-94.

70. Akrami FM, Ahmadi Z, Hassanshahi G, et al. (2013) Dose Ramadan fasting affects inflammatory responses: evidences for modulatory roles of this nique nutritional status via chemokine network. Iran J Basic Med Sci 16, 1217-1222.

71. Norouzy A, Salehi M, Philippou E, et al. (2013) Effect of fasting in Ramadan on body composition and nutritional intake: a prospective study. J Human Nutr Diets 26, 97-104.

72. Pirsaheb S, Pasdar Y, Navabi SJ, et al. (2013) Fasting consequences during Ramadan on lipid profile and dietary patterns. J Nutr Fasting Health 1, 6-12.

73. Rohin MAK, Rozano N, Abd Hadi N, et al. (2013) Anthropometry and body composition status during Ramadan among higher institution learning centre staffs with different body weight status. Sci World J 2013, 308041.

74. Rabiee S, Afghari N \& Rastmanesh R (2014) Short-term and mid-term effects of fasting and downset meal pattern on lipid profile in Iranian fasted women. $J$ Religion Health 53, 654-657.

75. Akaberi A, Golshan A, Moojdekanloo M, et al. (2014) Does fasting in Ramadan ameliorate Lipid profile? A prospective observational study. PakJ Med Sci 30, 708-711.

76. Akhtaruzzaman M, Hoque N, Choudhury M, et al. (2014) Effect of Ramadan fasting on serum lipid profile of Bangladeshi female volunteers. Bangladesh J Med Biochem 7, 47-51.

77. AlNahari H \& Kouja H (2014) Impact of Ramadan fasting on some biochemical aspects in healthy subjects. Int $J$ Med Health Sci Res 1, 144-154.

78. Celik A, Saricicek E, Saricicek V, et al. (2014) Effect of Ramadan fasting on serum concentration of apelin-13 and new obesity indices in healthy adult men. Med Sci Monit 20, 337 .

79. Feizollahzadeh S, Rasuli J, Kheirouri S, et al. (2014) Augmented plasma adiponectin after prolonged fasting during Ramadan in men. Health Promot Perspec 4, 77-81.

80. Hassan MB \& Isawumi MA (2014) Effects of fasting on intraocular pressure in a black population. Middle East Afr J Ophthalmol 21, 328-331.

81. Ismail WI \& Haron N (2014) Effect of Ramadan fasting on serum lipid profile among healthy students in UiTM. Int Conf Biol Chemical Environ Sci, 51-53.

82. McNeil J, Mamlouk MM, Duval K, et al. (2014) Alterations in metabolic profile occur in normal-weight and obese men during the Ramadan fast despite no changes in anthropometry. J Obesity 2014, 482547.

83. Salahuddin M, Masood-ul-Hassan J \& Javed MH (2014) Effects of Ramadan fasting on some physiological and biochemical parameters in healthy and hypertensive subjects in Aurangabad district of Maharashtra, India. J Fasting Health 2, 7-13.

84. Pathan M \& Patil R (2015) Effect of Ramadan fasting on body weight and lipid profile. Biomed Pharmacol J 3, $167-170$.

85. Ara T, Jahan N, Sultana N, et al. (2015) Effect of Ramadan fasting on total cholesterol (TC) low density lipoprotein cholesterol (LDL-C) and high density lipoprotein cholesterol (HDL-C) in healthy adult male. J Bangladesh Soc Physiol 10, 46-50.

86. Reza S, Hosseini SRA \& Hejazi K (2015) Evaluation of changes in blood hematological and biochemical parameters in response to Islamic fasting and regular physical activity in male and female subjects. J Nutr Fasting Health 3, 118-125.

87. López-Bueno M, González-Jiménez E, Navarro-Prado S, et al. (2015) Influence of age and religious fasting on the body composition of Muslim women living in a westernized context. Nutr Hosp 31, 1067-1073.

88. Shahsavan F, Askarizadeh F, Namaty M, et al. (2015) Effect of Ramadan fasting on blood pressure and lipid profiles. J Nutr Fasting Health 3, 126-131.

89. Suriani I, Shamsuddin K, Latif KA, et al. (2015) The effect of the Malaysian food guideline guidance on a group of overweight and obese women during Ramadan. Saudi Med J 36, 40-45.

90. Babaei AA, Ghasemi M, Ghouchani HT, et al. (2016) Evaluation of the effects of Islamic fasting on the biochemical markers of health. J Fasting Health 4, 88-91.

91. BaHammam AS, Pandi-Perumal SR \& Alzoghaibi MA (2016) The effect of Ramadan intermittent fasting on lipid peroxidation in healthy young men while controlling for diet and sleep: a pilot study. Ann Thorac Med 11, 43-48.

92. Esmaeilzadeh F \& van de Borne P (2016) Does intermittent fasting improve microvascular endothelial function in healthy middle-aged subjects? Biol Med (Aligarb) 8, 337.

93. Ganjali N, Mozaffari-Khosravi H, Afkhami Ardakani M, et al. (2016) Effect of islamic fasting on glucose, lipid profiles and body mass index, adiponectin and leptin in obese individuals. J Nutr Fasting Health 4, 57-63.

94. Sezen Y, Altiparmak IH, Erkus ME, et al. (2016) Effects of Ramadan fasting on body composition and arterial stiffness. J Pak Med Assoc 66, 1522-1527.

95. Kiyani MM, Memon AR, Amjad MI, et al. (2017) Study of human Biochemical parameters during and after Ramadan. J Religion Health 56, 55-62.

96. AbdulKareem NG, Khalil NS, Jasim AE, et al. (2017) Some biochemical changes during summer Islamic fasting in diseased patients in comparison with normal. Am J Med Sci 5, 27-34.

97. Alsubheen SAA, Ismail M, Baker A, et al. (2017) The effects of diurnal Ramadan fasting on energy expenditure and substrate oxidation in healthy men. Br J Nutr 118, 1023-1030.

98. Bakki B, Goni BW \& HarunaYusuph FB (2017) Ramadan fasting: effect on the metabolic profile of healthy medical students in North East, Nigeria. Saudi J Med 6, 133-137.

99. Khan N, Rasheed A, Ahmed H, et al. (2017) Effect of Ramadan fasting on glucose level, lipid profile, HbA1c and uric acid among medical students in Karachi, Pakistan. East Mediter Health J 23, 274-279.

100. Malekmakan L, Sayadi M, Pakfetrat M, et al. (2017) The effect of fasting on anthropometric parameters and blood pressure levels: a report from Southern Iran. Int Cardiovasc Res J 11, 143-147.

101. Norouzy A, Hasanzade Daloee M, Khoshnasab AH, et al. (2017) Trend of blood pressure in hypertensive and normotensive volunteers during Ramadan fasting. Blood Pressure Monit 22, 253-257.

102. Ongsara S, Boonpol S, Prompalad N, et al. (2017) The effect of Ramadan fasting on biochemical parameters in healthy Thai subjects. J Clin Diagn Res 11, BC14-BC18.

103. Mohammadzade F, Vakili M, Seyediniaki A, et al. (2017) Effect of prolonged intermittent fasting in Ramadan on biochemical and inflammatory parameters of healthy men. JClin Basic Res 1, 38-46.

104. Abubakar M, Hassan A, Saddique MU, et al. (2018) Comparison of sugar level and blood lipids while fasting in 1st and 4th week of Holy month of Ramadhan at services hospital, Lahore. Indo Am J Pharm Sci 5, 5693-5697. 
105. Al-Barha NS \& Aljaloud KS (2018) The effect of Ramadan fasting on body composition and metabolic syndrome in apparently healthy men. Am J Men Health 13, 1557988318816925.

106. Prasetya G \& Sapwarobol S (2018) Intermittent fasting during Ramadan improves insulin sensitivity and anthropometric parameters in healthy young Muslim men. Am J Lifestyle Med, https://doi.org/10.1177\%2F1559827618815430

107. Rahbar AR, Safavi E, Rooholamini M, et al. (2019) Effects of intermittent fasting during Ramadan on insulin-like growth factor-1, interleukin 2, and lipid profile in healthy Muslims. Int Prev Med 15, 7

108. Higgins JPT, Thompson SG, Deeks JJ, et al. (2003) Measuring inconsistency in meta-analyses. BMJ 327, 557-560.

109. Higgins JPT, Thomas J, Chandler J, et al. (editors) (2019) Cochrane Handbook for Systematic Reviews of Interventions, 2nd ed. Chichester: John Wiley \& Sons.

110. Azzoug S, Mahgoun S \& Chentli F (2015) Diabetes mellitus and Ramadan in elderly patients. I Pak Med Assoc 65, S33-S36.

111. Reddy Kilim S \& Chandala SR (2013) A comparative study of lipid profile and oestradiol in pre- and post-menopausal women. J Clin Diagn Res 7, 1596-1598.

112. Fernandez ML \& Murillo AG (2016) Postmenopausal women have higher HDL and decreased incidence of low HDL than premenopausal women with metabolic syndrome. Healthcare (Basel) 4, 20.

113. Patterson RE, Laughlin GA, Lacroix AZ, et al. (2015) Intermittent fasting and human metabolic health. $J$ Acad Nutr Diet 115, 1203-1212.

114. Patterson RE \& Sears DD (2017) Metabolic effects of intermittent fasting. Ann Rev Nutr 37, 371-393.

115. Trepanowski JF \& Bloomer RJ (2010) The impact of religious fasting on human health. Nutr J9, 57.

116. Trepanowski JF, Canale RE, Marshall KE, et al. (2011) Impact of caloric and dietary restriction regimens on markers of health and longevity in humans and animals: a summary of available findings. Nutr J 10, 107.

117. Hajer GR, van der Graaf Y, Olijhoek JK, et al. (2007) Low plasma levels of adiponectin are associated with low risk for future cardiovascular events in patients with clinical evident vascular disease. Am Heart J 154, 750.e1-750.e7.

118. Isomaa B, Almgren P, Tuomi T, et al. (2001) Cardiovascular morbidity and mortality associated with the metabolic syndrome. Diabetes Care 24, 683-689.

119. Vardarli MC, Hammes H-P \& Vardarli I (2014) Possible metabolic impact of Ramadan fasting in healthy men. Turk J Med Sci 44, 1010-1020.

120. Hosseini SRA, Sardar MA, Hejazi K, et al. (2015) The effects of aerobic exercise during Ramadan on the levels of leptin and adiponectin in overweight women. J Fasting Health 3, $35-42$.

121. Mushtaq R, Akram A, Mushtaq R, et al. (2019) The role of inflammatory markers following Ramadan fasting. Pak Med Sci 35, 77-81

122. Faris M, Madkour MI, Obaideen AK, et al. (2019) Effect of Ramadan diurnal fasting on visceral adiposity and serum adipokines in overweight and obese individuals. Diabetes Res Clin Pract 153, 166-175.

123. Sarraf-Zadegan N, Atashi M, Naderi GA, et al. (2000) The effect of fasting in Ramadan on the values and interrelations between biochemical, coagulation and hematological factors. Ann Saudi Med 20, 377-381.

124. Saleh S, El-Kemery T, Farrag K, et al. (2004) Ramadan fasting: relation to atherogenic risk among obese Muslims. J Egypt Public Health Assoc 79, 461-483.

125. Nahid B, Sadegh A, Amin A, et al. (2013) The effect of regular aerobic exercise on the FBS, HOMA indexes and lipid profiles in non-active obese men; with emphasis on Ramadan fasting. Int J Med Pharm Sci 3, 31-38.

126. Zare A, Hajhashemi M, Hassan ZM, et al. (2011) Effect of Ramadan fasting on serum heat shock protein 70 and serum lipid profile. Singap Med J 52, 491-495.

127. Yousefi B, Faghfoori Z, Samadi N, et al. (2014) The effects of Ramadan fasting on endothelial function in patients with cardiovascular diseases. Eur J Clin Nutr 68, 835-839.

128. Daiber A, Xia N, Steven S, et al. (2019) New therapeutic implications of endothelial nitric oxide synthase (eNOS) function/dysfunction in cardiovascular disease. Int Mol Sci 20, 187.

129. Soeki T \& Sata M (2016) Inflammatory biomarkers and atherosclerosis. Int Heart J 57, 134-139.

130. Libby P, Ridker PM \& Hansson GK (2009) Inflammation in atherosclerosis. J Am Coll Cardiol 54, 2129-2138.

131. Tangvarasittichai S, Pongthaisong S \& Tangvarasittichai O (2016) Tumor necrosis factor-A, interleukin-6, C-reactive protein levels and insulin resistance associated with type 2 diabetes in abdominal obesity women. Indian J Clin Biochem 31, 68-74.

132. Madkour MI, El-Serafi A, Jahrami HA, et al. (2019) Ramadan diurnal intermittent fasting modulates SOD2, TFAM, Nrf2, and sirtuins (SIRT1, SIRT3) gene expressions in subjects with overweight and obesity. Diabetes Res Clin Prac 155, 107801.

133. Grundy SM (1998) Hypertriglyceridemia, atherogenic dyslipidemia, and the metabolic syndrome. Am J Cardiol 81, 18B-25B

134. McCracken E, Monaghan M \& Sreenivasan S (2018) Pathophysiology of the metabolic syndrome. Clin Dermat 36, 14-20.

135. Radhakishun N, Blokhuis C, van Vliet M, et al. (2014) Intermittent fasting during Ramadan causes a transient increase in total, LDL, and HDL cholesterols and hs-CRP in ethnic obese adolescents. Eur J Pediatr 173, 1103-1106.

136. Nematy M, Alinezhad-Namaghi M, Rashed MM, et al. (2012) Effects of Ramadan fasting on cardiovascular risk factors: a prospective observational study. Nutr J 11, 69.

137. Syam AF, Sobur CS, Abdullah M, et al. (2016) Ramadan fasting decreases body fat but not protein mass. Int J Endocrinol Metab 14, e29687.

138. Harder-Lauridsen NM, Rosenberg A, Benatti FB, et al. (2017) Ramadan model of intermittent fasting for $28 \mathrm{~d}$ had no major effect on body composition, glucose metabolism, or cognitive functions in healthy lean men. Nutrition 37, 92-103.

139. Gamble KL, Berry R, Frank SJ, et al. (2014) Circadian clock control of endocrine factors. Nat Rev Endocrinol 10, 466-475.

140. Elnakib S (2017) Ramadan: the practice of fasting. https:// www.eatright.org/health/lifestyle/culture-and-traditions/ ramadan-the-practice-of-fasting (accessed March 2019). 